\title{
PSFC/JA-97-24 \\ Stability Properties of Coherently Gyrating Relativistic Electron Beams
}

\author{
J. A. Davies and C. Chen \\ November, 1997 \\ Plasma Science and Fusion Center \\ Massachusetts Institute of Technology \\ Cambridge, MA 02139, USA
}

This research was supported in part by Air Force Office of Scientific Research Grant Numbers F49620-94-1-0374 and F49620-97-1-0325. Reproduction, translation, publication, use and disposal, in whole or part, by or for the United States government is permitted.

Submitted for publication in Physics of Plasmas. 


\title{
Stability Properties of Coherently Gyrating Relativistic Electron Beams
}

\author{
J. A. Davies ${ }^{a)}$ and C. Chen \\ Plasma Science and Fusion Center \\ Massachusetts Institute of Technology \\ Cambridge, Massachusetts 02139
}

\begin{abstract}
An investigation is made of the effects of nongyrotropic equilibrium distributions in the phase angle of $p_{\perp}$ on the stability properties of a relativistic electron beam propagating along and gyrating about an applied uniform magnetic field. Perturbations are assumed to vary spatially only in the direction of the applied magnetic field, so that generated electromagnetic and longitudinal electric fields propagate parallel to the applied field. The two equilibrium distributions considered are the time-dependent distribution $f_{0}\left(p_{\perp}, p_{z}, \xi\right)$ with $\xi=\phi-\Omega_{c} t / \gamma$ and the axial-dependent distribution $f_{0}\left(p_{\perp}, p_{z}, \zeta\right)$ with $\zeta=\phi-$ $m \Omega_{c} z / p_{z}$. A Vlasov-Maxwell analysis leads to integral equations relating the field Fourier components. These equations reduce to algebraic equations when no spread in $\gamma$ is present in the time-dependent equilibrium distribution and when no spread in $p_{z}$ is present in the axial-dependent distribution. Numerical computations for these special cases show that a rich variety of stability properties are obtained by changing the distributions in $\xi$ and $\zeta$.
\end{abstract}

a) Permanent Address: Department of Physics, Clark University, Worcester, Massachusetts 01610 


\section{Introduction}

During the past two decades, extensive studies have been made of the stability properties of a relativistic electron beam propagating along an applied uniform magnetic field $B_{0} \hat{\mathbf{e}}_{\mathbf{z}}{ }^{1-11}$ If the beam possesses a population inversion associated with the component of momentum perpendicular to the field $\left(p_{\perp}\right)$, then this system may be subject to the whistler and cyclotron-resonance maser instabilities, which initiate the process of converting electron-beam kinetic energy into coherent electromagnetic radiation. The cyclotronresonance maser instability provides the basis for existing and proposed electronic amplifiers and oscillators such as the gyrotron and the cyclotron autoresonance maser. ${ }^{12-19}$ With the inclusion of thermal background electrons in addition to the beam, this instability is of interest in such problems in space- and astrophysics ${ }^{20-28}$ as the generation of auroral kilometric and Jovian decametric radiation.

In this paper, we carry out a stability analysis of this system (exclusive of an ambient thermal background) using Vlasov-Maxwell theory. We make the assumption that the spatial variation of all quantities is in the $z$-direction only; however, the electron beam is not assumed to be gyrotropic in the phase angle $\phi$ of the component of the particle momentum normal to the $z$-axis. Most previous analyses of this system assume that the equilibrium distribution is of the form $f_{0}\left(p_{\perp}, p_{z}\right)$, i.e. that the beam is gyrotropic. Analyses of the nongyrotropic case are limited in number. Using the eikonal approximation, Fruchtman and Friedland ${ }^{7,8}$ have considered the case of a stationary amplifier with a nongyrotropic equilibrium distribution of the form $f_{0}\left(p_{\perp}, p_{z}, \zeta\right)=p_{\perp}^{-1} \delta\left(p_{\perp}-p_{\perp 0}\right) \delta\left(p_{z}-p_{z 0}\right) g(\zeta)$, where $\zeta=\phi-m \Omega_{c} z / p_{z}$ and $\Omega_{c}$ is the nonrelativistic cyclotron frequency. Kho, et al., ${ }^{9}$ using the same equilibrium distribution, have dropped the assumption of a stationary amplifier but assume that the left-hand polarized perturbed radiation field can be ignored. Chen, et $a l .^{18}$ consider $\mathrm{TE}$ modes for the case of a helical relativistic electron beam in a cylindrical waveguide. In an application to space plasmas, Freund, et al. ${ }^{22}$ consider the case of a diffuse electron beam propagating in a cold magnetized ambient plasma. The beam is coherent in phase, and has a thermal spread in $p_{\perp}$ but no spread in $p_{z}$.

Two equilibrium distributions are introduced in Sec. II. These are the time-dependent 
distribution $f_{0}\left(p_{\perp}, p_{z}, \xi\right)$ where $\xi=\phi-\Omega_{c} t / \gamma$ and the spatial-dependent distribution $f_{0}\left(p_{\perp}, p_{z}, \zeta\right)$ where $\zeta=\phi-m \Omega_{c} z / p_{z}$. By manipulating Fourier transforms of the VlasovMaxwell equations, we obtain sets of equations relating components of the perturbed right- and left-hand polarized electromagnetic and the electrostatic fields. For the timedependent equilibrium, these relations are given in Eqs. (26)-(28). The relations for the spatial-dependent equilibrium distribution appear in Eqs. (41)-(43). In either case, these relations are integral equations, not algebraic equations. Eqs. (26)-(28) reduce algebraic equations only if there is no spread in $p$ (i.e., in $\gamma$ ) in the time-dependent equilibrium distribution. Moreover, Eqs. (41)-(43) reduce to algebraic equations only if there is no spread in $p_{z}$ in the spatial-dependent equilibrium distribution.

The analysis of these integral equations is the subject of current research and is not dealt with further in this paper. Instead, in the remainder of the paper, we deal with cases in which the integral equations reduce to algebraic equations.

Stability properties for the case of the time-dependent equilibrium distribution with no spread in $p$ are considered in Sec. III. Most generally, spreads in the pitch angle $\alpha=\tan ^{-1}\left(p_{\perp} / p_{z}\right)$ and the phase angle $\phi$ may be present. In this case, the integral equations (26)-(28) reduce to just three algebraic relations presented in Eq. (58) of Sec. III.A. The corresponding exact dispersion relation, relating complex frequencies and complex wave numbers, is the three by three determinant relation in Eq. (64). In Sec. III.B, we consider the more restrictive case of no spread in the pitch angle $\alpha$ in the equilibrium distribution. (Then, both $p_{\perp}$ and $p_{z}$ have definite equilibrium values.) In this case, the dispersion relation in Eq. (64) reduces to the tenth-degree polynomial relation in Eq. (69). Numerical computations of growth-rate curves (Im $\hat{\omega}$ vs. $\hat{k}$, real) and properties of eigenmodes of Eq. (58) are presented in Sec. III.C for the case of definite equilibrium $p_{\perp}$ and $p_{z}$ and various equilibrium distributions in $\phi$.

An analogous treatment is given in Sec. IV for the case of the axial-dependent equilibrium distribution with no spread in $p_{z}$. Spreads in $p_{\perp}$ and $\phi$ are still permitted. For this case, it is shown in Sec. IV.A that the integral equations (41)-(43) reduce to the three algebraic relations in Eq. (89). The exact dispersion relation for the system is given by the three by three determinant equation (95). In Sec. IV.B, the additional condition that 
there be no equilibrium spread in $p_{\perp}$ is imposed. Then, (as in the time-dependent case) the dispersion relation reduces to the tenth-degree polynomial equation (100) relating the complex frequency and complex wave number. Numerical computations of growth-rate curves and properties of the eigenmodes of Eq. (89) for this case are presented in Sec. IV.C. A summary of our results and conclusions is presented in Sec. V. 


\section{Formulation of the Problem}

A beam consists of relativistic electrons, which propagate along and gyrate about a uniform magnetic field $\mathbf{B}_{\mathbf{0}}=B_{0} \hat{\mathbf{e}}_{\mathbf{z}}$. Initially the beam is in an equilibrium state in which temporally and spatially varying electromagnetic fields are absent. The initial growth rates of these fields are obtained by regarding them as small perturbations on the equilibrium. The system is treated as one-dimensional in the sense that the spatial variation of all variables is in the $z$-direction only. Consequently, only electromagnetic waves propagating parallel or anti-parallel to the uniform field $\mathbf{B}_{\mathbf{0}}$ are included in this analysis. Furthermore, equilibrium self fields are assumed to be negligibly small, so that results discussed below are limited to the case of a small ratio of the plasma frequency to the cyclotron frequency. ${ }^{6}$

\section{A. Equilibrium Distributions}

A single-particle momentum $\mathbf{p}$ can be described by the components $p_{\perp}, p_{z}$, and $\phi$, where $\phi$ is the phase angle (as shown in Fig. 1). This paper deals with two systems in which the phase angle $\phi$ is not necessarily random in the equilibrium distribution. One simple constant of the single-particle motion involving $\phi$ is $\xi=\phi-\frac{\Omega_{c}}{\gamma} t$, where $\Omega_{c}=$ $e B_{0} / m c$ is the nonrelativistic electron cyclotron frequency, $-e$ and $m$ are the electron charge and rest mass, respectively, $c$ is the speed of light in vacuo, $t$ is the time, and $\gamma=\left(1+p^{2} / m^{2} c^{2}\right)^{1 / 2}=\left(1+p_{\perp}^{2} / m^{2} c^{2}+p_{z}^{2} / m^{2} c^{2}\right)^{1 / 2}$ is the relativistic mass factor of the electron. An equilibrium distribution of the form

$$
f_{0}(\mathbf{p}, t)=f_{0}\left(p_{\perp}, p_{z}, \xi\right)
$$

where

$$
\xi=\phi-\frac{\Omega_{c}}{\gamma} t
$$

corresponds to an equilibrium electron beam that is homogenous in the configuration space at any given time. Since the equilibrium distribution (1) is nonstationary in the momentum space, we refer to it as the time-dependent equilibrium distribution. 
Another simple constant of the single-particle motion involving $\phi$ is

$$
\zeta=\phi-\frac{\Omega_{c}}{\gamma} \frac{z}{v_{z}}=\phi-m \frac{\Omega_{c}}{p_{z}} z
$$

where $\mathbf{v}$ is the electron velocity. Use of $\zeta$ gives rise to an alternative equilibrium distribution

$$
f_{0}(z, \mathbf{p})=f_{0}\left(p_{\perp}, p_{z}, \zeta\right)
$$

In this case, the equilibrium distribution is constant in time at any given $z$. It is analogous to a typical laboratory situation where the beam is introduced into the interaction region at some initial $z=0$ with a given distribution in $\phi$. Then, if interactions with the electromagnetic field are excluded, the electrons move along the field lines with constant $v_{z}$ and gyrate around them with the constant relativistic cyclotron frequency $\Omega_{c} / \gamma$. Consequently, the phase at any value of $z$ is the phase at $z=0$ plus $\Omega_{c} z / \gamma v_{z}$. We refer to this distribution as the axial-dependent equilibrium distribution.

The time- and axial- dependent distributions are illustrated with examples in Fig. 2. It is shown in the Appendix that the two distributions in Eqs. (1) and (4) are physically different in the sense that neither can be transformed into the other by a Lorentz transformation.

In the remainder of this section, we derive linearized equations relating components of the perturbed electromagnetic fields for each of the distributions in Eqs. (1) and (4).

\section{B. Perturbation Analysis for the Time-Dependent Equilibrium Distribution.}

The equilibrium distribution is of the form in Eq. (1), i.e., $f_{0}(\mathbf{p}, t)=f_{0}\left(p_{\perp}, p_{z}, \xi\right)=$ $f_{0}\left(p_{\perp}, p_{z}, \phi-\Omega_{c} t / \gamma\right)$. The distribution is assumed to be periodic in $\xi$ and (for fixed $\left.t\right)$ in $\phi$ with period $2 \pi$. Consequently, the normalization of $f_{0}\left(p_{\perp}, p_{z}, \phi-\Omega_{c} t / \gamma\right)$ over momentum space is time-independent and is defined by

$$
\int_{-\infty}^{\infty} d p_{z} \int_{0}^{\infty} d p_{\perp} \int_{0}^{2 \pi} d \phi p_{\perp} f_{0}\left(p_{\perp}, p_{z}, \phi-\frac{\Omega_{c}}{\gamma} t\right)=1
$$

As time progresses, the distribution evolves under perturbations into

$$
f(z, \mathbf{p}, t)=f_{0}(\mathbf{p}, t)+f_{1}(z, \mathbf{p}, t),
$$


where $f_{1}(z, \mathbf{p}, t)$ is considered to be a small perturbation. To first order in the perturbation, the time evolution of this quantity is governed by the linearized Vlasov equation,

$$
\begin{gathered}
\left.\frac{d f_{1}}{d t}\right|_{0}=\frac{\partial}{\partial t} f_{1}(z, \mathbf{p}, t)+v_{z} \frac{\partial}{\partial z} f_{1}(z, \mathbf{p}, t)-\frac{e}{c} \mathbf{v} \times \mathbf{B}_{0} \cdot \nabla_{\mathbf{p}} f_{1}(z, \mathbf{p}, t) \\
=e\left(\mathbf{E}_{1}+\frac{\mathbf{v} \times \mathbf{B}_{1}}{\mathbf{c}}\right) \cdot \nabla_{\mathbf{p}} f_{0}\left(p_{x}, p_{y}, p_{z}, t\right)
\end{gathered}
$$

where the total time derivative is along a characteristic of the unperturbed motion (i.e., $z(t+\tau)=z+v_{z} \tau, p_{z}(t+\tau)=p_{z}, p_{\perp}(t+\tau)=p_{\perp}, \phi(t+\tau)=\phi+\Omega_{c} \tau / g a m m a$, and the variable of integration $\tau$ is in the range from $-\infty$ to 0$)$. The fields $\mathbf{E}_{1}(z, t)$ and $\mathbf{B}_{1}(z, t)$ are regarded as small perturbations governed by Maxwell's equations, which in the present treatment reduce to

$$
\begin{aligned}
\frac{\partial^{2}}{\partial z^{2}} E_{1 \pm}(z, t)-\frac{1}{c^{2}} \frac{\partial^{2}}{\partial t^{2}} E_{1 \pm}(z, t) & =\frac{4 \pi}{c^{2}} \frac{\partial}{\partial t} J_{1 \pm}(z, t), \\
\frac{\partial}{\partial z} E_{1 \pm}(z, t) & = \pm \frac{i}{c} \frac{\partial}{\partial t} B_{1 \pm}(z, t), \\
\frac{\partial E_{1 z}}{\partial z}(z, t) & =4 \pi \rho_{1}(z, t), \\
B_{1 z}(z, t) & =0 .
\end{aligned}
$$

In the above equations:

$$
\begin{aligned}
E_{1 \pm}(z, t) & =E_{1 x}(z, t) \pm i E_{1 y}(z, t), \\
B_{1 \pm}(z, t) & =B_{1 x}(z, t) \pm i B_{1 y}(z, t), \\
J_{1 \pm}(z, t) & =-e n_{0} \int d^{3} p f_{1}(z, \mathbf{p}, t) \frac{p_{ \pm}}{\gamma m}, \\
\rho_{1}(z, t) & =-e n_{0} \int d^{3} p f_{1}(z, \mathbf{p}, t), \\
p_{ \pm} & =\gamma m v_{ \pm}=p_{\perp} \exp ( \pm i \phi),
\end{aligned}
$$

where $n_{0}$ is the mean electron number density. The fields $\left(E_{1-}, B_{1-}\right)$ and $\left(E_{1+}, B_{1+}\right)$ represent right- and left-hand circularly polarized transverse electromagnetic waves, respectively, whereas the field $\left(E_{1 z}, B_{1 z}=0\right)$ describes longitudinal (electrostatic) waves.

Because most of the integration of Eq. (7) along characteristics is standard, not all of the details will be given here. We remark that before integrating, it is convenient to express 
the $\mathrm{x}$ - and $\mathrm{y}$-components of the vectors that appear in Eq. (7) in terms of $v_{ \pm}, E_{ \pm}$, and $B_{ \pm}$. Moreover, $\partial f_{0}\left(p_{x}, p_{y}, p_{z}, t\right) / \partial p_{x}, \partial f_{0}\left(p_{x}, p_{y}, p_{z}, t\right) / \partial p_{y}$, and $\partial f_{0}\left(p_{x}, p_{y}, p_{z}, t\right) / \partial p_{z}$ must be expressed in terms of $\partial f_{0}\left(p_{\perp}, p_{z}, \xi\right) / \partial p_{\perp}, \partial f_{0}\left(p_{\perp}, p_{z}, \xi\right) / \partial p_{z}, \partial f_{0}\left(p_{\perp}, p_{z}, \xi\right) / \partial \xi$, because the latter partial derivatives are constant on a characteristic. Once these constant derivatives are removed from the integral sign, the subsequent calculation of the Fourier transform of $f_{1}(z, \mathbf{p}, t)$ is facilitated by rewriting them in terms of $\partial f_{0}\left(p_{\perp}, p_{z}, \phi, t\right) / \partial p_{\perp}$, $\partial f_{0}\left(p_{\perp}, p_{z}, \phi, t\right) / \partial p_{z}, \partial f_{0}\left(p_{\perp}, p_{z}, \phi, t\right) / \partial \phi$, and $\partial f_{0}\left(p_{\perp}, p_{z}, \phi, t\right) / \partial t$. The expression obtained for $f_{1}(z, \mathbf{p}, t)$ is

$$
\begin{aligned}
& f_{1}\left(z, p_{\perp}, p_{z}, \phi, t\right)= \\
& \quad U\left(p_{\perp}, p_{z}, \phi, t\right) \int_{-\infty}^{0} d \tau \exp \left(i \frac{\Omega_{c}}{\gamma} \tau\right) E_{1-}\left(z+v_{z} \tau, t+\tau\right) \\
& \quad+\frac{e}{2} \exp (i \phi) \frac{p_{\perp} \Omega_{c}}{\gamma^{3} m^{2} c^{2}} \frac{\partial}{\partial \phi} f_{0}\left(p_{\perp}, p_{z}, \phi, t\right) \int_{-\infty}^{0} d \tau \tau \exp \left(i \frac{\Omega_{c}}{\gamma} \tau\right) E_{1-}\left(z+v_{z} \tau, t+\tau\right) \\
& \quad+V\left(p_{\perp}, p_{z}, \phi, t\right) \int_{-\infty}^{0} d \tau \exp \left(i \frac{\Omega_{c}}{\gamma} \tau\right) B_{1-}\left(z+v_{z} \tau, t+\tau\right) \\
& \quad+U^{*}\left(p_{\perp}, p_{z}, \phi, t\right) \int_{-\infty}^{0} d \tau \exp \left(-i \frac{\Omega_{c}}{\gamma} \tau\right) E_{1+}\left(z+v_{z} \tau, t+\tau\right) \\
& \quad+\frac{e}{2} \exp (-i \phi) \frac{p_{\perp} \Omega_{c}}{\gamma^{3} m^{2} c^{2}} \frac{\partial}{\partial \phi} f_{0}\left(p_{\perp}, p_{z}, \phi, t\right) \int_{-\infty}^{0} d \tau \tau \exp \left(-i \frac{\Omega_{c}}{\gamma} \tau\right) E_{1+}\left(z+v_{z} \tau, t+\tau\right) \\
& +V^{*}\left(p_{\perp}, p_{z}, \phi, t\right) \int_{-\infty}^{0} d \tau \exp \left(-i \frac{\Omega_{c}}{\gamma} \tau\right) B_{1+}\left(z+v_{z} \tau, t+\tau\right) \\
& +e \frac{\partial}{\partial p_{z}} f_{0}\left(p_{\perp}, p_{z}, \phi, t\right) \int_{-\infty}^{0} d \tau E_{1 z}\left(z+v_{z} \tau, t+\tau\right) \\
& +\frac{e p_{z} \Omega_{c}}{\gamma^{3} m^{2} c^{2}} \frac{\partial}{\partial \phi} f_{0}\left(p_{\perp}, p_{z}, \phi, t\right) \int_{-\infty}^{0} d \tau \tau E_{1 z}\left(z+v_{z} \tau, t+\tau\right) .
\end{aligned}
$$

In the above equation,

$$
\begin{aligned}
& U\left(p_{\perp}, p_{z}, \phi, t\right)=\frac{e}{2} \exp (i \phi)\left(\frac{\partial}{\partial p_{\perp}}+\frac{i}{p_{\perp}} \frac{\partial}{\partial \phi}\right) f_{0}\left(p_{\perp}, p_{z}, \phi, t\right) \\
& V\left(p_{\perp}, p_{z}, \phi, t\right)=\frac{e}{2} \frac{\exp (i \phi)}{\gamma m c}\left(-i p_{z} \frac{\partial}{\partial p_{\perp}}+i p_{\perp} \frac{\partial}{\partial p_{z}}+\frac{p_{z}}{p_{\perp}} \frac{\partial}{\partial \phi}\right) f_{0}\left(p_{\perp}, p_{z}, \phi, t\right) .
\end{aligned}
$$

In order to relate components of the perturbed electromagnetic fields, it is necessary to obtain Fourier (or Laplace) transforms of $f_{1}(z, \mathbf{p}, t)$ (Eq. (17)) and of Maxwell's equations (8)-(11). The simple assumption that all variables vary as $\exp [i(k z-\omega t)]$ leads to 
inconsistent results. In general, one cannot assume an infinite series of terms of the form $\exp \left\{i\left[k z-\left(\omega-n \Omega_{c} / \gamma\right) t\right]\right\}$ (where $n$ is an integer), because $\gamma$ is not defined if $f_{0}\left(p_{\perp}, p_{z}, \xi\right.$ ) contains an energy spread. In the present analysis, we define the spatial and temporal Fourier transforms by

$$
\begin{aligned}
& F(k, \omega)=\frac{1}{2 \pi} \int_{-\infty}^{\infty} d z \int_{-\infty}^{\infty} d t F(z, t) \exp [i(\omega t-k z)] \\
& F(z, t)=\frac{1}{2 \pi} \int_{-\infty}^{\infty} d k \int_{-\infty}^{\infty} d \omega F(k, \omega) \exp [i(k z-\omega t)]
\end{aligned}
$$

The calculation of the Fourier transform of $f_{1}(z, \mathbf{p}, t)$, denoted by $f_{1}(k, \mathbf{p}, \omega)$, requires use of the convolution theorem

$$
\left(F_{1} F_{2}\right)(k, \omega)=\frac{1}{2 \pi} \int_{-\infty}^{\infty} d k^{\prime} \int_{-\infty}^{\infty} d \omega^{\prime} F_{1}\left(k^{\prime}, \omega^{\prime}\right) F_{2}\left(k-k^{\prime}, \omega-\omega^{\prime}\right) .
$$

Then, using Eqs. (17)-(19), together with the Fourier transform of the Maxwell equation (9), and assuming that orders of multiple integrals can be interchanged, we obtain (after a lengthy calculation) the following expression for the Fourier transform:

$$
\begin{aligned}
& f_{1}(k, \mathbf{p}, \omega)= \\
& \frac{1}{2} \frac{e}{\sqrt{2 \pi}} \exp (i \phi) \int_{-\infty}^{+\infty} d \omega^{\prime}\left\{\left[\omega-\omega^{\prime}-k v_{z}-\frac{\Omega_{c}}{\gamma}\right]^{-1}\right. \\
& \quad \times\left[\frac{\omega-\omega^{\prime}-k v_{z}}{\omega-\omega^{\prime}}\left(i \frac{\partial}{\partial p_{\perp}}-\frac{1}{p_{\perp}} \frac{\partial}{\partial \phi}\right)+i \frac{p_{\perp}}{\gamma m c} \frac{c k}{\omega-\omega^{\prime}} \frac{\partial}{\partial p_{z}}\right] f_{0}\left(p_{\perp}, p_{z}, \phi, \omega^{\prime}\right) \\
& \left.+\left[\omega-\omega^{\prime}-k v_{z}-\frac{\Omega_{c}}{\gamma}\right]^{-2} \frac{p_{\perp} \Omega_{c}}{\gamma^{3} m^{2} c^{2}} \frac{\partial}{\partial \phi} f_{0}\left(p_{\perp}, p_{z}, \phi, \omega^{\prime}\right)\right\} E_{1-}\left(k, \omega-\omega^{\prime}\right) \\
& +\frac{1}{2} \frac{e}{\sqrt{2 \pi}} \exp (-i \phi) \int_{-\infty}^{+\infty} d \omega^{\prime}\left\{\omega-\omega^{\prime}-k v_{z}+\frac{\Omega_{c}}{\gamma}\right]^{-1} \\
& \quad \times\left[\frac{\omega-\omega^{\prime}-k v_{z}}{\omega-\omega^{\prime}}\left(i \frac{\partial}{\partial p_{\perp}}+\frac{1}{p_{\perp}} \frac{\partial}{\partial \phi}\right)+i \frac{p_{\perp}}{\gamma m c} \frac{c k}{\omega-\omega^{\prime}} \frac{\partial}{\partial p_{z}}\right] f_{0}\left(p_{\perp}, p_{z}, \phi, \omega^{\prime}\right) \\
& \left.+\left[\omega-\omega^{\prime}-k v_{z}+\frac{\Omega_{c}}{\gamma}\right]^{-2} \frac{p_{\perp} \Omega_{c}}{\gamma^{3} m^{2} c^{2}} \frac{\partial}{\partial \phi} f_{0}\left(p_{\perp}, p_{z}, \phi, \omega^{\prime}\right)\right\} E_{1+}\left(k, \omega-\omega^{\prime}\right) \\
& +\frac{e}{\sqrt{2 \pi}} \int_{-\infty}^{+\infty} d \omega^{\prime}\left\{\left[\omega-\omega^{\prime}-k v_{z}\right]^{-1} i \frac{\partial}{\partial p_{z}} f_{0}\left(p_{\perp}, p_{z}, \phi, \omega^{\prime}\right)\right. \\
& \left.+\left[\omega-\omega^{\prime}-k v_{z}\right]^{-2} \frac{p_{z} \Omega_{c}}{\gamma^{3} m^{2} c^{2}} \frac{\partial}{\partial \phi} f_{0}\left(p_{\perp}, p_{z}, \phi, \omega^{\prime}\right)\right\} E_{1 z}\left(k, \omega-\omega^{\prime}\right) .
\end{aligned}
$$


The Fourier transforms of the Maxwell equations (8) and (10) are

$$
\begin{aligned}
\left(\omega^{2}-c^{2} k^{2}\right) E_{1 \pm}(k, \omega) & =4 \pi i e n_{0} \omega \int_{0}^{\infty} d p_{\perp} \int_{-\infty}^{\infty} d p_{z} \int_{0}^{2 \pi} d \phi \frac{p_{\perp}^{2}}{\gamma m} \\
\times \exp ( \pm i \phi) f_{1}\left(k, p_{\perp}, p_{z}, \phi, \omega\right) & \\
k E_{1 z}(k, \omega) & =4 \pi i e n_{0} \int_{0}^{\infty} d p_{\perp} \int_{-\infty}^{\infty} d p_{z} \int_{0}^{2 \pi} d \phi p_{\perp} f_{1}\left(k, p_{\perp}, p_{z}, \phi, \omega\right)
\end{aligned}
$$

By referring to Eq. (20), it is seen that Eqs. (21) and (22) are a set of integral equations (with variable of integration $\omega^{\prime}$ ) relating the Fourier transforms of the fields $E_{1 \pm}$ and $E_{1 z}$.

Partial derivatives of $f_{0}\left(p_{\perp}, p_{z}, \phi, \omega^{\prime}\right)$ appear in the expression for $f_{1}(k, \mathbf{p}, \omega)$ in Eq. (20). These derivatives are removed from the integrands in Eqs. (21) and (22) by integrating by parts with respect to $p_{\perp}, p_{z}$, and $\phi$, employing the periodicity of $f_{0}\left(p_{\perp}, p_{z}, \phi, \omega^{\prime}\right)$ in $\phi$. The procedure is straight forward but requires much algebra.

The periodicity of $f_{0}\left(p_{\perp}, p_{z}, \xi\right)$ in $\xi$ (or $\phi$ ) has not yet been fully employed in this analysis. Expansion of $f_{0}\left(p_{\perp}, p_{z}, \xi\right)$ in a Fourier series gives

$$
f_{0}\left(p_{\perp}, p_{z}, \xi\right)=\frac{1}{\sqrt{2 \pi}} \sum_{n=-\infty}^{+\infty} g_{n}\left(p_{\perp}, p_{z}\right) \exp (i n \xi)
$$

where

$$
g_{n}\left(p_{\perp}, p_{z}\right)=\frac{1}{\sqrt{2 \pi}} \int_{0}^{2 \pi} d \xi f_{0}\left(p_{\perp}, p_{z}, \xi\right) \exp (-i n \xi)
$$

Because $f_{0}\left(p_{\perp}, p_{z}, \xi\right)$ is real, $g_{n}^{*}\left(p_{\perp}, p_{z}\right)=g_{-n}\left(p_{\perp}, p_{z}\right)$. From Eq. (23), we see that the temporal Fourier transform of $f_{0}\left(p_{\perp}, p_{z}, \xi\right)=f_{0}\left(p_{\perp}, p_{z}, \phi, t\right)$ is given by

$$
f_{0}\left(p_{\perp}, p_{z}, \phi, \omega\right)=\sum_{n=-\infty}^{+\infty} g_{n}\left(p_{\perp}, p_{z}\right) \exp (i n \phi) \delta\left(\omega-n \frac{\Omega_{c}}{\gamma}\right)
$$

With the aid of Eq. (25), the integrations over $\omega^{\prime}$ can be carried out in Eqs. (21) and (22). Moreover, with the aid of the relation $\int_{0}^{2 \pi} d \phi \exp (i n \phi)=2 \pi \delta_{n 0}$, the integrals over $\phi$ can also be completed. In fact, only the $n=0,1$, and -1 terms of the infinite series in Eq. (25) contribute to the right-hand sides of Eqs. (21) and (22). In the nonrelativistic limit of $\gamma=1$, the integral equations in (21) and (22) reduce to algebraic equations relating 
the Fourier transforms of the fields $E_{1 \pm}$ and $E_{1 z}$ because of the Dirac delta function in Eq. (25). However, in the general case, $\gamma=\left(p^{2} / m^{2} c^{2}+1\right)^{1 / 2}=\left[\left(p_{\perp}^{2}+p_{z}^{2}\right) / m^{2} c^{2}+1\right]^{1 / 2}$. Consequently, Eqs. (21) and (22) remain integral equations in the two variables $p_{\perp}$ and $p_{z}$, relating such unknown functions as $E_{1-}\left(k, \omega+2 \Omega_{c} / \gamma\left(p_{\perp}, p_{z}\right)\right), E_{1 z}\left(k, \omega+\Omega_{c} / \gamma\left(p_{\perp}, p_{z}\right)\right)$, and $E_{1+}(k, \omega)$.

Eqs. (21) and (22) can be reduced to a set of integral equations in the single variable $p$ by replacing the variables $p_{\perp}$ and $p_{z}$ with the new variables $p$ and $\alpha$, where $\alpha=\tan ^{-1}\left(p_{\perp} / p_{z}\right)$ is the pitch angle shown in Fig. 1. Setting $d p_{\perp} d p_{z}=p d p d \alpha$ in the equations obtained from Eqs. (21) and (22), we obtain the following set of simultaneous integral equations relating the Fourier transforms of the perturbed fields $E_{1 \pm}$ and $E_{1 z}$ :

$$
\begin{aligned}
D_{--}\left(k, \omega, \Omega_{c}\right) E_{1-}(k, \omega)= & \int_{0}^{\infty} d p p^{2} \chi_{-+}\left(k, \omega, \Omega_{c}, p\right) E_{1+}\left(k, \omega-\frac{2 \Omega_{c}}{\gamma(p)}\right) \\
& +\int_{0}^{\infty} d p p^{2} \chi_{-z}\left(k, \omega, \Omega_{c}, p\right) E_{1 z}\left(k, \omega-\frac{\Omega_{c}}{\gamma(p)}\right), \\
D_{++}\left(k, \omega, \Omega_{c}\right) E_{1+}(k, \omega)= & \int_{0}^{\infty} d p p^{2} \chi_{+-}\left(k, \omega, \Omega_{c}, p\right) E_{1-}\left(k, \omega+\frac{2 \Omega_{c}}{\gamma(p)}\right) \\
& +\int_{0}^{\infty} d p p^{2} \chi_{+z}\left(k, \omega, \Omega_{c}, p\right) E_{1 z}\left(k, \omega+\frac{\Omega_{c}}{\gamma(p)}\right), \\
D_{z z}(k, \omega) E_{1 z}(k, \omega)= & \int_{0}^{\infty} d p p^{2} \chi_{z+}\left(k, \omega, \Omega_{c}, p\right) E_{1+}\left(k, \omega-\frac{\Omega_{c}}{\gamma(p)}\right) \\
& +\int_{0}^{\infty} d p p^{2} \chi_{z-}\left(k, \omega, \Omega_{c}, p\right) E_{1-}\left(k, \omega+\frac{\Omega_{c}}{\gamma(p)}\right) .
\end{aligned}
$$

In the above integral equations:

$$
\begin{aligned}
D_{--}\left(k, \omega, \Omega_{c}\right)=\omega^{2} & -c^{2} k^{2}-\frac{\sqrt{2 \pi}}{2} \Omega_{p}^{2} \int_{0}^{\infty} d p \int_{0}^{\pi} d \alpha p g_{0}(p, \alpha) \\
& \times\left[\frac{2 p \sin \alpha}{\gamma}\left(\omega-\frac{k p \cos \alpha}{\gamma m}\right)\left(\omega-\frac{k p \cos \alpha}{\gamma m}-\frac{\Omega_{c}}{\gamma}\right)^{-1}\right. \\
& \left.-\frac{p^{3} \sin ^{3} \alpha}{\gamma^{3} m^{2} c^{2}}\left(\omega^{2}-c^{2} k^{2}\right)\left(\omega-\frac{k p \cos \alpha}{\gamma m}-\frac{\Omega_{c}}{\gamma}\right)^{-2}\right]
\end{aligned}
$$




$$
\begin{aligned}
& D_{++}\left(k, \omega, \Omega_{c}\right)=D_{--}\left(k, \omega,-\Omega_{c}\right) \\
& D_{z z}(k, \omega)=1-\sqrt{2 \pi} \Omega_{p}^{2} \int_{0}^{\infty} d p \int_{0}^{\pi} d \alpha p^{2} g_{0}(p, \alpha) \frac{\sin \alpha}{\gamma} \\
& \times\left(1-\frac{p^{2} \cos ^{2} \alpha}{\gamma^{2} m^{2} c^{2}}\right)\left(\omega-\frac{k p \cos \alpha}{\gamma m}\right)^{-2}, \\
& \chi_{-+}\left(k, \omega, \Omega_{c}, p\right)=-\frac{\sqrt{2 \pi}}{2} \Omega_{p}^{2} \omega \int_{0}^{\pi} d \alpha p^{2} g_{2}(p, \alpha) \frac{\sin ^{3} \alpha}{\gamma^{3} m^{2} c^{2}}\left(\omega-\frac{2 \Omega_{c}}{\gamma}\right)^{-1} \\
& \times\left(\omega^{2}-2 \omega \frac{\Omega_{c}}{\gamma}-c^{2} k^{2}\right)\left(\omega-\frac{k p \cos \alpha}{\gamma m}-\frac{\Omega_{c}}{\gamma}\right)^{-2} \\
& \chi_{+-}\left(k, \omega, \Omega_{c}, p\right)=\chi_{-+}^{*}\left(k^{*}, \omega^{*},-\Omega_{c}, p\right) \\
& \chi_{-z}\left(k, \omega, \Omega_{c}, p\right)=-\sqrt{2 \pi} \omega \Omega_{p}^{2} \int_{0}^{\pi} d \alpha p g_{1}(p, \alpha) \frac{\sin ^{2} \alpha}{\gamma^{2} m c} \\
& \times\left(\frac{\omega p \cos \alpha}{\gamma m c}-c k\right)\left(\omega-\frac{k p \cos \alpha}{\gamma m}-\frac{\Omega_{c}}{\gamma}\right)^{-2} \text {, } \\
& \chi_{+z}\left(k, \omega, \Omega_{c}, p\right)=\chi_{-z}^{*}\left(k^{*}, \omega^{*},-\Omega_{c}, p\right) \\
& \chi_{z-}\left(k, \omega, \Omega_{c}, p\right)=-\frac{\sqrt{2 \pi}}{2} \Omega_{p}^{2}\left(\omega+\frac{\Omega_{c}}{\gamma}\right)^{-1} \int_{0}^{\pi} d \alpha p g_{-1}(p, \alpha) \frac{\sin ^{2} \alpha}{\gamma^{2} m c} \\
& \times\left[\frac{p \cos \alpha}{\gamma m c}\left(\omega+\frac{\Omega_{c}}{\gamma}\right)-c k\right]\left(\omega-\frac{k p \cos \alpha}{\gamma m}\right)^{-2} \\
& =\frac{1}{2}\left(\omega+\frac{\Omega_{c}}{\gamma}\right)^{-2} \chi_{-z}^{*}\left(k^{*}, \omega^{*}+\frac{\Omega_{c}}{\gamma}, \Omega_{c}, p\right) \text {, } \\
& \chi_{z+}\left(k, \omega, \Omega_{c}, p\right)=\chi_{z-}^{*}\left(k^{*}, \omega^{*},-\Omega_{c}, p\right)
\end{aligned}
$$

Here, $\Omega_{p}=\left(4 \pi n_{0} e^{2} / m\right)^{1 / 2}$ is the nonrelativistic plasma frequency.

Notice that the structure of Eqs. (26)-(28) is a coupling of $E_{1-}\left(k, \omega^{\prime}\right)$, $E_{z}\left(k, \omega^{\prime}-\frac{\Omega_{c}}{\gamma(p)}\right)$, and $E_{1+}\left(k, \omega^{\prime}-\frac{2 \Omega_{c}}{\gamma(p)}\right)$ over the range of $p$ for which the equilibrium distribution is nonvanishing.

\section{Perturbation Analysis for the Axial-Dependent Equilibrium Distribution.}

The analysis for the case of the equilibrium distribution in Eq. (4), i.e., $f_{0}(z, \mathbf{p})=$ $f_{0}\left(p_{\perp}, p_{z}, \zeta\right)=f_{0}\left(p_{\perp}, p_{z}, \phi-m \Omega_{c} z / p_{z}\right)$ is similar to the analysis of the previous section. 
The distribution is assumed periodic in $\zeta$ and (for fixed $z$ ) in $\phi$ with period $2 \pi$. Consequently, the normalization of $f_{0}\left(p_{\perp}, p_{z}, \phi-m \frac{\Omega_{c}}{p_{z}} z\right)$ over momentum space is independent of $z$. It is defined to be

$$
\int_{-\infty}^{\infty} d p_{z} \int_{0}^{\infty} d p_{\perp} \int_{0}^{2 \pi} d \phi p_{\perp} f_{0}\left(p_{\perp}, p_{z}, \phi-m \frac{\Omega_{c}}{p_{z}} z\right)=1
$$

The linearized Vlasov equation for the system is the same as Eq. (7) except that the factor $f_{0}\left(p_{x}, p_{y}, p_{z}, t\right)$ on the right-hand side is to be replaced with $f_{0}\left(z, p_{x}, p_{y}, p_{z}\right)$. Maxwell's equations (8)-(16) are applicable without modification. The derivation of integral equations relating the Fourier transforms of the perturbed fields involves a great deal of algebra but closely parallels that given in Sec. II.B for the spatially-homogenous equilibrium distribution. Consequently, we omit the details of the derivation. We remark that in the present derivation $z$ plays much the same role as $t$ in the previous derivation and $k$ much the same role as $\omega$. Periodicity of $f_{0}\left(p_{\perp}, p_{z}, \zeta\right)$ in $\zeta$ gives rise to the Fourier series expansion

$$
f_{0}\left(p_{\perp}, p_{z}, \zeta\right)=\frac{1}{\sqrt{2 \pi}} \sum_{n=-\infty}^{+\infty} h_{n}\left(p_{\perp}, p_{z}\right) \exp (i n \zeta)
$$

where

$$
h_{n}\left(p_{\perp}, p_{z}\right)=\frac{1}{\sqrt{2 \pi}} \int_{0}^{2 \pi} d \zeta f_{0}\left(p_{\perp}, p_{z}, \zeta\right) \exp (-i n \zeta) .
$$

For the z-dependent equilibrium distribution $f_{0}\left(p_{\perp}, p_{z}, \phi-m \Omega_{c} z / p_{z}\right)$, the integral equations are

$$
\begin{aligned}
D_{--}^{\prime}\left(k, \omega, \Omega_{c}\right) E_{1-}(k, \omega)= & \int_{-\infty}^{\infty} d p_{z} \eta_{-+}\left(k, \omega, \Omega_{c}, p_{z}\right) E_{1+}\left(k+\frac{2 m \Omega_{c}}{p_{z}}, \omega\right) \\
& +\int_{-\infty}^{\infty} d p_{z} \eta_{-z}\left(k, \omega, \Omega_{c}, p_{z}\right) E_{1 z}\left(k+\frac{m \Omega_{c}}{p_{z}}, \omega\right), \\
D_{++}^{\prime}\left(k, \omega, \Omega_{c}\right) E_{1+}(k, \omega)= & \int_{-\infty}^{\infty} d p_{z} \eta_{+-}\left(k, \omega, \Omega_{c}, p_{z}\right) E_{1-}\left(k-\frac{2 m \Omega_{c}}{p_{z}}, \omega\right) \\
& +\int_{-\infty}^{\infty} d p_{z} \eta_{+z}\left(k, \omega, \Omega_{c}, p_{z}\right) E_{1 z}\left(k-\frac{m \Omega_{c}}{p_{z}}, \omega\right), \\
D_{z z}^{\prime}(k, \omega) E_{1 z}(k, \omega)= & \int_{-\infty}^{\infty} d p_{z} \eta_{z-}\left(k, \omega, \Omega_{c}, p_{z}\right) E_{1-}\left(k-\frac{m \Omega_{c}}{p_{z}}, \omega\right) \\
& +\int_{-\infty}^{\infty} d p_{z} \eta_{z+}\left(k, \omega, \Omega_{c}, p_{z}\right) E_{1+}\left(k+\frac{m \Omega_{c}}{p_{z}}, \omega\right) .
\end{aligned}
$$


In the above integral equations:

$$
\begin{aligned}
& D_{--}^{\prime}\left(k, \omega, \Omega_{c}\right)=\omega^{2}-c^{2} k^{2}-\frac{\sqrt{2 \pi}}{2} \Omega_{p}^{2} \int_{-\infty}^{\infty} d p_{z} \int_{0}^{\infty} d p_{\perp} h_{0}\left(p_{\perp}, p_{z}\right) \frac{p_{\perp}}{\gamma} \\
& \times\left[2\left(\omega-\frac{k p_{z}}{\gamma m}\right)\left(\omega-\frac{k p_{z}}{\gamma m}-\frac{\Omega_{c}}{\gamma}\right)^{-1}\right. \\
& \left.-\frac{p_{\perp}^{2}}{\gamma^{2} m^{2} c^{2}}\left(\omega^{2}-c^{2} k^{2}\right)\left(\omega-\frac{k p_{z}}{\gamma m}-\frac{\Omega_{c}}{\gamma}\right)^{-2}\right] \\
& D_{++}^{\prime}\left(k, \omega, \Omega_{c}\right)=D_{--}^{\prime}\left(k, \omega,-\Omega_{c}\right) \\
& D_{z z}^{\prime}(k, \omega)=1-\sqrt{2 \pi} \Omega_{p}^{2} \int_{-\infty}^{\infty} d p_{z} \int_{0}^{\infty} d p_{\perp} p_{\perp} h_{0}\left(p_{\perp}, p_{z}\right) \gamma^{-1} \\
& \times\left(1-\frac{p_{z}^{2}}{\gamma^{2} m^{2} c^{2}}\right)\left(\omega-\frac{k p_{z}}{\gamma m}\right)^{-2} \\
& \eta_{-+}\left(k, \omega, \Omega_{c}, p_{z}\right)=\frac{\sqrt{2 \pi}}{2} \Omega_{p}^{2} \int_{0}^{\infty} d p_{\perp} h_{2}\left(p_{\perp}, p_{z}\right) \frac{p_{\perp}^{3}}{\gamma^{3} m^{2} c^{2}} \\
& \times\left[-\omega^{2}+c^{2} k\left(k+\frac{2 m \Omega_{c}}{p_{z}}\right)\right]\left(\omega-\frac{k p_{z}}{\gamma m}-\frac{\Omega_{c}}{\gamma}\right)^{-2}, \\
& \eta_{+-}\left(k, \omega, \Omega_{c}, p_{z}\right)=\eta_{-+}^{*}\left(k^{*}, \omega^{*},-\Omega_{c}, p_{z}\right), \\
& \eta_{-z}\left(k, \omega, \Omega_{c}, p_{z}\right)=\sqrt{2 \pi} \Omega_{p}^{2} \omega \int_{0}^{\infty} d p_{\perp} h_{1}\left(p_{\perp}, p_{z}\right) \frac{p_{\perp}^{2}}{\gamma^{3} m^{2} c^{2}} \\
& \times\left(-\omega p_{z}+k \gamma m c^{2}\right)\left(\omega-\frac{k p_{z}}{\gamma m}-\frac{\Omega_{c}}{\gamma}\right)^{-2}, \\
& \eta_{+z}\left(k, \omega, \Omega_{c}, p_{z}\right)=\eta_{-z}^{*}\left(k^{*}, \omega^{*},-\Omega_{c}, p_{z}\right), \\
& \eta_{z-}\left(k, \omega, \Omega_{c}, p_{z}\right)=\frac{\sqrt{2 \pi}}{2} \frac{\Omega_{p}^{2}}{k} \int_{0}^{\infty} d p_{\perp} h_{-1}\left(p_{\perp}, p_{z}\right) \frac{p_{\perp}^{2}}{\gamma^{2} m c^{2}} \\
& \times\left[-\frac{k p_{z}}{\gamma m}+\frac{c^{2} k}{\omega}\left(k-\frac{m \Omega_{c}}{p_{z}}\right)\right]\left(\omega-\frac{k p_{z}}{\gamma m}\right)^{-2}, \\
& \eta_{z+}\left(k, \omega, \Omega_{c}, p_{z}\right)=\eta_{z-}^{*}\left(k^{*}, \omega^{*},-\Omega_{c}, p_{z}\right) .
\end{aligned}
$$

The structure of Eqs. (41)-(43) is the coupling of $E_{1-}\left(\hat{k}^{\prime}, \omega\right)$ to $E_{1 z}\left(\hat{k}^{\prime}+m \Omega_{c} / p_{z}, \omega\right)$ and $E_{1+}\left(\hat{k}^{\prime}+2 m \Omega_{c} / p_{z}, \omega\right)$ over the range of $p_{z}$ for which the equilibrium distribution is nonvanishing. 


\section{Some Special Cases.}

In general Eqs. (26)-(28) [Eqs. (41)-43)] couple the right- and left-hand circularly polarized radiation fields $E_{ \pm}$and the longitudinal, relativistic, plasma wave field $E_{z}$. However, if $g_{ \pm 1}\left(p_{\perp}, p_{z}\right)=g_{ \pm 1}(p, \alpha)=0\left[h_{ \pm 1}\left(p_{\perp}, p_{z}\right)=0\right]$, then Eq. (28) decouples from Eqs. (26) and (27) [Eq. (43) decouples from Eqs. (41) and (42)] to yield the dispersion relation for longitudinal plasma oscillations. The radiation fields remain coupled, as is seen in Eqs. (36) and (37) [Eqs. (51) and (52)]. From Eq. (24) [Eq. (40)], it is seen that this situation occurs whenever the Fourier series for $f_{0}\left(p_{\perp}, p_{z}, \phi-\frac{\Omega_{c}}{\gamma} t\right)\left[f_{0}\left(p_{\perp}, p_{z}, \phi-\frac{m \Omega_{c}}{p_{z}} z\right)\right]$ contains neither $\cos \phi$ - nor $\sin \phi$-components.

If $g_{ \pm 2}\left(p_{\perp}, p_{z}\right)=g_{ \pm 2}(p, \alpha)=0\left[h_{ \pm 2}\left(p_{\perp}, p_{z}\right)=0\right]$ then all three fields $\left(E_{ \pm}\right.$and $\left.E_{z}\right)$ remain coupled, however the radiation fields couple only through the electrostatic oscillations and not directly with each other. From Eq. (24) [Eq. (40)], it is seen that this situation occurs whenever the Fourier series for $f_{0}\left(p_{\perp}, p_{z}, \phi-\frac{\Omega_{c}}{\gamma} t\right)\left[f_{0}\left(p_{\perp}, p_{z}, \phi-\frac{m \Omega_{c}}{p_{z}} z\right)\right]$ contains neither $\cos 2 \phi$ - nor $\sin 2 \phi$-components.

Finally, if $g_{ \pm 1}\left(p_{\perp}, p_{z}\right)=g_{ \pm 2}\left(p_{\perp}, p_{z}\right)=0\left[h_{ \pm 1}\left(p_{\perp}, p_{z}\right)=h_{ \pm 2}\left(p_{\perp}, p_{z}\right)=0\right]$, then Eqs. (26)-(28) [Eqs. (41)-43)] decouple completely and reduce to the dispersion relations

$$
\begin{aligned}
D_{--}\left(k, \omega, \Omega_{c}\right) & =0, \\
D_{--}\left(k, \omega, \Omega_{c}\right) & =0, \\
D_{z z}(k, \omega) & =0 .
\end{aligned}
$$

These dispersion relations are identical to those for the case in which the distribution in $\phi$ is uniformly random. Referring to Eqs. (24) and (40), we see that $g_{0}\left(p_{\perp}, p_{z}\right)=$ $\frac{1}{\sqrt{2 \pi}} \int_{0}^{2 \pi} d \xi f_{0}\left(p_{\perp}, p_{z}, \xi\right)$ and $h_{0}\left(p_{\perp}, p_{z}\right)=\frac{1}{\sqrt{2 \pi}} \int_{0}^{2 \pi} d \eta f_{0}\left(p_{\perp}, p_{z}, \eta\right)$. For the case of a uniformly random distribution in $\phi\left(i . e_{.}, f_{0}\left(p_{\perp}, p_{z}\right)\right)$, both of these expressions reduce to $g_{0}\left(p_{\perp}, p_{z}\right)=h_{0}\left(p_{\perp}, p_{z}\right)=\sqrt{2 \pi} f_{0}\left(p_{\perp}, p_{z}\right)$. For this case either Eqs. (26) and (27) or Eqs. (41) and (42) reduce to the dispersion relations for the cyclotron resonance maser with random phase obtained by Chu and Hirshfield. ${ }^{1}$

The analysis of Eqs. (26)-(28) [(41)-43)] as integral equations is the subject of present research and results of the analysis will be presented in a subsequent paper. However, 
many important special cases exist in which the equations reduce to algebraic equations from which dispersion relations can be derived. Some of these cases will be analyzed in the remainder of this paper. 


\section{Dispersion Characteristics for the Time-Dependent Equilibrium Distribu- tion without Energy Spread.}

In the previous section, it was noted that, for a uniformly random equilibrium distribution in $\phi$, equations (26)-(28) decouple and reduce to the well known dispersion relations in Eqs. (53)-(55). In this section it is shown that as long as there is no energy spread in the equilibrium distribution in Eq. (1), the integral equations (26)-(28) reduce to algebraic relations between the Fourier components of the fields even when the distributions are not uniform in $\phi$. The dispersion characteristics are illustrated with numerical examples for the time-dependent equilibrium distribution with no spread in $p_{\perp}$ or $p_{z}$.

\section{A. Analysis}

For a beam with a definite energy $\gamma\left(p_{0}\right) m c^{2} \equiv \gamma_{0} m c^{2}=\left(p_{0}^{2} c^{2}+m^{2} c^{4}\right)^{1 / 2}$ the most general distribution in Eq. (1) is of the form

$$
f_{0}(p, \alpha, \xi)=\frac{1}{p^{2}} \delta\left(p-p_{0}\right) \hat{f}_{0}\left(p_{0}, \alpha, \xi\right)
$$

where $\xi=\phi-\Omega_{c} t / \gamma$. From Eq. (24), the coeficients in the Fourier series expansion of this distribution are

$$
\begin{gathered}
g_{n}(p, \alpha)=\frac{1}{p^{2}} \delta\left(p-p_{0}\right) \hat{g}_{n}\left(p_{0}, \alpha\right) \\
\hat{g}_{n}\left(p_{0}, \alpha\right)=\frac{1}{\sqrt{2 \pi}} \int_{0}^{2 \pi} d \xi \hat{f}_{0}\left(p_{0}, \alpha, \xi\right) \exp (-i n \xi)
\end{gathered}
$$

Substitute Eq. (57) into Eqs. (29)-(37) and then substitute the results into the integral equations (26)-(28). After replacing $\omega$ with $\omega-2 \Omega_{c} / \gamma_{0}$ in Eq. (27) and with $\omega-\Omega_{c} / \gamma_{0}$ in Eq. (28), we obtain three homogeneous algebraic equations relating just three field components. Expressed in matrix notation, these equations are

$$
\mathrm{DE}=0
$$


where

$\mathbf{D}=\left(\begin{array}{ccc}D_{-}\left(k, \omega, \Omega_{c}, p_{0}\right) & -\hat{\chi}_{-+}\left(k, \omega, \Omega_{c}, p_{0}\right) & -\hat{\chi}_{-z}\left(k, \omega, \Omega_{c}, p_{0}\right) \\ -\hat{\chi}_{+-}\left(k, \omega-\frac{2 \Omega_{c}}{\gamma_{0}}, \Omega_{c}, p_{0}\right) & D_{++}\left(k, \omega-\frac{2 \Omega_{c}}{\gamma_{0}}, \Omega_{c}, p_{0}\right) & -\hat{\chi}_{+z}\left(k, \omega-\frac{2 \Omega_{c}}{\gamma_{0}}, \Omega_{c}, p_{0}\right) \\ -\hat{\chi}_{z-}\left(k, \omega-\frac{\Omega_{c}}{\gamma_{0}}, \Omega_{c}, p_{0}\right) & -\hat{\chi}_{z+}\left(k, \omega-\frac{\Omega_{c}}{\gamma_{0}}, \Omega_{c}, p_{0}\right) & D_{z z}\left(k, \omega-\frac{\Omega_{c}}{\gamma_{0}}, p_{0}\right)\end{array}\right)$,

and

$$
\mathbf{E}=\left(\begin{array}{c}
E_{1-}(k, \omega) \\
E_{1+}\left(k, \omega-\frac{2 \Omega_{c}}{\gamma_{0}}\right) \\
E_{1 z}\left(k, \omega-\frac{\Omega_{c}}{\gamma_{0}}\right)
\end{array}\right)
$$

The quantities $\hat{\chi}_{i j}$ appearing in the matrix in Eq. (59) are obtained from the corresponding quantities in Eqs. (32)-(37) simply by replacing each $g_{n}(p, \alpha)$ with $\hat{g}_{n}\left(p_{0}, \alpha\right)$ and setting $p=p_{0}$ and $\gamma=\gamma_{0}$. Moreover, from Eqs. (29)-(31), the diagonal matrix elements can be expressed as

$$
\begin{aligned}
D_{--}\left(k, \omega, \Omega_{c}, p_{0}\right)= & \omega^{2}-c^{2} k^{2}-\frac{\sqrt{2 \pi}}{2} \Omega_{p}^{2} \int_{0}^{\pi} d \alpha \hat{g}_{0}\left(p_{0}, \alpha\right) \\
& \times\left[\frac{2 \sin \alpha}{\gamma_{0}}\left(\omega-\frac{k p_{0} \cos \alpha}{\gamma_{0} m}\right)\left(\omega-\frac{k p_{0} \cos \alpha}{\gamma_{0} m}-\frac{\Omega_{c}}{\gamma_{0}}\right)^{-1}\right. \\
& \left.-\frac{p_{0}^{2} \sin ^{3} \alpha}{\gamma_{0}^{3} m^{2} c^{2}}\left(\omega^{2}-c^{2} k^{2}\right)\left(\omega-\frac{k p_{0} \cos \alpha}{\gamma_{0} m}-\frac{\Omega_{c}}{\gamma_{0}}\right)^{-2}\right] \\
D_{++}\left(k, \omega, \Omega_{c}, p_{0}\right)= & \hat{D}_{--}\left(k, \omega,-\Omega_{c}, p_{0}\right), \\
D_{z z}\left(k, \omega, p_{0}\right)=1 & -\sqrt{2 \pi} \Omega_{p}^{2} \int_{0}^{\pi} d \alpha \hat{g}_{0}\left(p_{0}, \alpha\right) \frac{\sin \alpha}{\gamma_{0}} \\
& \times\left(1-\frac{p_{0}^{2} \cos ^{2} \alpha}{\gamma_{0}^{2} m^{2} c^{2}}\right)\left(\omega-\frac{k p_{0} \cos \alpha}{\gamma_{0} m}\right)^{-2} .
\end{aligned}
$$

Recall that, $\Omega_{p}=\left(4 \pi e^{2} n_{0} / m\right)^{1 / 2}$ is the nonrelativistic plasma frequency.

The dispersion relation for this system is

$$
\operatorname{det} \mathbf{D}(k, \omega)=0
$$

From Eq. (60), it is seen that, for a given value of the wavenumber $k$, the frequency $\omega$ is that of the right-hand polarized wave $E_{1-}$. The corresponding frequencies of the 
left-hand polarized wave $E_{1+}$ and of the electrostatic wave $E_{1 z}$ are $\omega-2 \Omega_{c} / \gamma_{0}$ and $\omega-$ $\Omega_{c} / \gamma_{0}$, respectively. We point out that, once the assumption of one-dimensional spatial dependence is made and equilibrium self fields are neglected, the dispersion relation is exact for equilibrium distributions of definite energy.

Again notice why, in the general case, the field components are related by integral equations (26)-(28) instead of algebraic equations. If the distribution $f_{0}\left(p, \alpha, \phi-\Omega_{c} t / \gamma(p)\right)$ is nonvanishing over a continuous range of energies $\gamma(p) m c^{2}$, then the mode $E_{1-}(k, \omega)$ will be coupled to a continuum of modes $E_{1+}\left(k, \omega-2 \Omega_{c} / \gamma(p)\right)$ and $E_{1 z}\left(k, \omega-\Omega_{c} / \gamma(p)\right)$.

\section{B. Case of Definite $p_{\perp}$ and $p_{z}$.}

As numerical examples, we consider equilibria of definite $p_{\perp}=p_{0} \sin \alpha_{0}$ and $p_{z}=$ $p_{0} \cos \alpha_{0}$. Consequently, the factor $\hat{f}_{0}\left(p_{0}, \alpha, \xi\right)$ in Eq. (56) is

$$
\hat{f}_{0}\left(p_{0}, \alpha, \xi\right)=\frac{\delta\left(\alpha-\alpha_{0}\right)}{\sin \alpha} \Phi(\xi)
$$

where $\Phi(\xi)$ is a function of period $2 \pi$. From Eq. (5), the normalization condition on $\Phi(\xi)$ is

$$
\int_{0}^{2 \pi} d \phi \Phi\left(\phi-\frac{\Omega_{c}}{\gamma} t\right)=\int_{0}^{2 \pi} d \xi \Phi(\xi)=1 .
$$

Moreover, from Eq. (57),

$$
\hat{g}_{n}\left(p_{0}, \alpha\right)=\frac{1}{\sqrt{2 \pi}} \frac{\delta\left(\alpha-\alpha_{0}\right)}{\sin \alpha} s_{n}
$$

where

$$
s_{n}=\int_{0}^{2 \pi} d \xi \Phi(\xi) \exp (-i n \xi) .
$$

From Eq. (66), $s_{0}=1$. Also notice that $s_{-n}=s_{n}^{*}$.

Substituting Eq. (67) into Eqs. (61)-(63) and into Eqs. (29)-(31) with $g_{n}(p, \alpha)$ replaced by $\hat{g}_{n}\left(p_{o}, \alpha\right)$, we can express the dispersion relation in Eq. (64) as the following tenth degree polynomial equation (in either $\hat{\omega}$ or $\hat{k}$ ) with real coefficients:

$$
M_{--}(\hat{k}, \hat{\omega}) M_{++}(\hat{k}, \hat{\omega}) M_{z z}(\hat{k}, \hat{\omega})=
$$




$$
\begin{aligned}
& \frac{1}{2}\left(\frac{\omega_{p}^{2}}{\omega_{c}^{2}}\right)^{2} \beta_{\perp}^{2}\left|s_{1}\right|^{2}\left[\left(\beta_{z} \hat{\omega}-\hat{k}\right)^{2} M_{++}(\hat{k}, \hat{\omega})+\left(\beta_{z} \hat{\omega}-2 \beta_{z}-\hat{k}\right)^{2} M_{--}(\hat{k}, \hat{\omega})\right] \\
& +\frac{1}{4}\left(\frac{\omega_{p}^{2}}{\omega_{c}^{2}}\right)^{2} \beta_{\perp}^{4}\left|s_{2}\right|^{2}\left(\hat{\omega}^{2}-2 \hat{\omega}-\hat{k}^{2}\right)^{2} M_{z z}(\hat{k}, \hat{\omega}) \\
& -\frac{1}{4}\left(\frac{\omega_{p}^{2}}{\omega_{c}^{2}}\right)^{3} \beta_{\perp}^{4}\left(s_{2} s_{-1}^{2}+s_{-2} s_{1}^{2}\right)\left(\hat{\omega}^{2}-2 \hat{\omega}-\hat{k}^{2}\right)\left(\beta_{z} \hat{\omega}-2 \beta_{z}-\hat{k}\right)\left(\beta_{z} \hat{\omega}-\hat{k}\right) .
\end{aligned}
$$

In Eq. (69), $\omega_{p}^{2}=\Omega_{p}^{2} / \gamma_{0}$ is the relativistic plasma frequency squared, and $\omega_{c}=\Omega_{c} / \gamma_{0}$ is the relativistic cyclotron frequency. Dimensionless frequencies and wavenumbers are $\hat{\omega}=\omega / \omega_{c}$ and $\hat{k}=c k / \omega_{c}$. Dimensionless velocities are given by $\beta_{\perp}=v_{\perp 0} / c$ and $\beta_{z}=v_{z 0} / c$, where $v_{\perp 0}=p_{0} \sin \alpha_{0} / \gamma\left(p_{0}\right) m$ and $v_{z 0}=p_{0} \cos \alpha_{0} / \gamma\left(p_{0}\right) m$. Finally,

$$
\begin{aligned}
& M_{--}(\hat{k}, \hat{\omega})=\left(\hat{\omega}^{2}-\hat{k}^{2}\right)\left(\hat{\omega}-\hat{k} \beta_{z}-1\right)^{2}-\frac{\omega_{p}^{2}}{\omega_{c}^{2}}\left(\hat{\omega}-\hat{k} \beta_{z}\right)\left(\hat{\omega}-\hat{k} \beta_{z}-1\right) \\
& \quad+\frac{1}{2} \frac{\omega_{p}^{2}}{\omega_{c}^{2}} \beta_{\perp}^{2}\left(\hat{\omega}^{2}-\hat{k}^{2}\right) \\
& M_{++}(\hat{k}, \hat{\omega})=\left((\hat{\omega}-2)^{2}-\hat{k}^{2}\right)\left(\hat{\omega}-\hat{k} \beta_{z}-1\right)^{2}-\frac{\omega_{p}^{2}}{\omega_{c}^{2}}\left(\hat{\omega}-\hat{k} \beta_{z}-2\right)\left(\hat{\omega}-\hat{k} \beta_{z}-1\right) \\
& \quad+\frac{1}{2} \frac{\omega_{p}^{2}}{\omega_{c}^{2}} \beta_{\perp}^{2}\left((\hat{\omega}-2)^{2}-\hat{k}^{2}\right) \\
& M_{z z}(\hat{k}, \hat{\omega})=\left(\hat{\omega}-\hat{k} \beta_{z}-1\right)^{2}-\frac{\omega_{p}^{2}}{\omega_{c}^{2}}\left(1-\beta_{z}^{2}\right) .
\end{aligned}
$$

The dispersion relation in Eq. (69), which is valid for both complex $\hat{\omega}$ and complex $\hat{k}$, is invariant under the transformation

$$
\begin{aligned}
& \hat{k} \longrightarrow-\hat{k}^{*}, \\
& \hat{\omega} \longrightarrow-\hat{\omega}^{*}+2 .
\end{aligned}
$$

For the case of the distribution in Eq. (65), the behavior of the eigenmode $\mathbf{E}$ in Eq. (58) under this transformation is easily determined by applying the transformation to the elements of $\mathbf{D}$ and $\mathbf{E}$ in Eq. (58). If either $s_{1} \neq 0$ or $s_{2} \neq 0$, then

$$
\frac{E_{1+}(\hat{k}, \hat{\omega}-2)}{E_{1-}(\hat{k}, \hat{\omega})} \longrightarrow \frac{E_{1+}\left(-\hat{k}^{*},-\hat{\omega}^{*}\right)}{E_{1-}\left(-\hat{k}^{*},-\hat{\omega}^{*}+2\right)}=\frac{E_{1-}^{*}(\hat{k}, \hat{\omega})}{E_{1+}^{*}(\hat{k}, \hat{\omega}-2)},
$$


and, if $s_{1} \neq 0$

$$
\begin{aligned}
& \frac{E_{1+}(\hat{k}, \hat{\omega}-2)}{E_{1 z}(\hat{k}, \hat{\omega}-1)} \rightarrow \frac{E_{1+}\left(-\hat{k}^{*},-\hat{\omega}^{*}\right)}{E_{1 z}\left(-\hat{k}^{*},-\hat{\omega}^{*}+1\right)}=\frac{E_{1-}^{*}(\hat{k}, \hat{\omega})}{E_{1 z}^{*}(\hat{k}, \hat{\omega}-1)}, \\
& \frac{E_{1-}(\hat{k}, \hat{\omega})}{E_{1 z}(\hat{k}, \hat{\omega}-1)} \rightarrow \frac{E_{1-}\left(-\hat{k}^{*},-\hat{\omega}^{*}+2\right)}{E_{1 z}\left(-\hat{k}^{*},-\hat{\omega}^{*}+1\right)}=\frac{E_{1+}^{*}(\hat{k}, \hat{\omega}-2)}{E_{1 z}^{*}(\hat{k}, \hat{\omega}-1)} .
\end{aligned}
$$

Equation (69) gives the ten branches of the dispersion relation $\hat{\omega}(\hat{k})$. The behavior of $\hat{\omega} / \hat{k}$ for large $|\hat{k}|$ is easily determined for each of these branches. As $|\hat{k}| \rightarrow \infty, \hat{\omega}(\hat{k}) / \hat{k} \rightarrow+1$ for two branches, $\hat{\omega}(\hat{k}) / \hat{k} \rightarrow-1$ for two branches, and $\hat{\omega}(\hat{k}) / \hat{k} \rightarrow \beta_{z}$ for six branches.

Simple expressions are easily obtained for the large $|\hat{k}|$ behaviors of $\hat{\omega}(\hat{k})$ for all ten branches if either $s_{1}$ or $s_{2}$ vanishes in Eq. (68). If $s_{1}=0$ and $s_{2} \neq 0$, then [from Eqs. (57) and (67)] $g_{1}(p, \alpha)=0$ and $g_{2}(p, \alpha) \neq 0$. It follows from the discussion in Sec. II.D that the electromagnetic components are coupled and the electrostatic component is uncoupled. [Such a situation holds for (but is not exclusive to) the distribution $\Phi(\xi)$ in Fig. 3(b), provided that the parameter $a \neq \pi$. For this distribution, $s_{1}=0$ and $s_{2}=\sin a / a$.] Two of the branches pertain to the uncoupled electrostatic waves and obey the exact dispersion relations

$$
\hat{\omega}=\hat{k} \beta_{z}+1 \pm \frac{\omega_{p}}{\omega_{c}}\left(1-\beta_{z}^{2}\right)^{1 / 2}
$$

for sufficiently large $|\hat{k}|$, the remaining eight branches obey the approximate dispersion relations

$$
\begin{aligned}
& \hat{\omega} \simeq \hat{k} \beta_{z}+1 \pm \frac{i}{\sqrt{2}} \frac{\omega_{p}}{\omega_{c}} \beta_{\perp}\left(1+\left|s_{2}\right|\right)^{1 / 2} \\
& \hat{\omega} \simeq \hat{k} \beta_{z}+1 \pm \frac{i}{\sqrt{2}} \frac{\omega_{p}}{\omega_{c}} \beta_{\perp}\left(1-\left|s_{2}\right|\right)^{1 / 2} \\
& \hat{\omega} \simeq \pm\left(\hat{k}^{2}+\frac{\omega_{p}^{2}}{\omega_{c}^{2}}\right)^{1 / 2} \\
& \hat{\omega} \simeq 2 \pm\left(\hat{k}^{2}+\frac{\omega_{p}^{2}}{\omega_{c}^{2}}\right)^{1 / 2}
\end{aligned}
$$


For real $\hat{k}$, Eqs. (77) and (78) give two branches with positive $\operatorname{Im} \hat{\omega}$ provided that $\left|s_{2}\right|<1$. In this case, growth-rate curves ( $\operatorname{Im} \hat{\omega}$ vs. real $\hat{k}$ ) will show two unstable branches at large $\hat{k}$.

If $s_{1} \neq 0$ and $s_{2}=0$, then [from Eqs. (57) and (67)] $g_{2}(p, \alpha)=0$ and $g_{1}(p, \alpha) \neq 0$. It follows from the discussion in Sec. II.D that the transverse electromagnetic waves are coupled through the longitudinal electrostatic wave. [A nonexclusive example of a distribution $\Phi(\xi)$ having this property (if $K \neq 0$ ) is presented in Fig. 3(c). Using Eq. (68), we find that $s_{1}=-4 i K$ and $s_{2}=0$ for this example.] In this case, the large $|\hat{k}|$ approximations for four of the ten branches of the dispersion relation are the same as those given in Eqs. (79) and (80) for the distribution previous case. Approximations for the remaining six branches are

$$
\begin{aligned}
& \hat{\omega} \simeq \hat{k} \beta_{z}+1 \pm \frac{i}{\sqrt{2}} \frac{\omega_{p}}{\omega_{c}} \beta_{\perp}, \\
& \hat{\omega} \simeq \hat{k} \beta_{z}+1 \pm \frac{1}{\sqrt{2}} \frac{\omega_{p}}{\omega_{c}} \kappa_{+}, \\
& \hat{\omega} \simeq \hat{k} \beta_{z}+1 \pm \frac{i}{\sqrt{2}} \frac{\omega_{p}}{\omega_{c}} \kappa_{-},
\end{aligned}
$$

where

$$
\kappa_{ \pm}=\left\{\mp\left(\frac{\beta_{\perp}^{2}}{2}-\left(1-\beta_{z}^{2}\right)\right)+\left[\left(\frac{\beta_{\perp}^{2}}{2}-\left(1-\beta_{z}^{2}\right)\right)^{2}+4 \beta_{\perp}^{2}\left(1-\beta_{z}^{2}\right)\left(\frac{1}{2}-\left|s_{1}\right|^{2}\right)\right]^{\frac{1}{2}}\right\}^{\frac{1}{2}} .
$$

[The maximum possible value of $K$ in Fig. 3(c) is $1 / 2 \pi$, and the corresponding maximum value of $\left|s_{1}\right|$ is $2 / \pi=0.6366 \ldots$. It is evident from Eq. (84) that the $\kappa_{ \pm}$are real and positive for all $\left|s_{1}\right|<1 / \sqrt{2}=0.7071 \ldots$. Consequently, Eqs. (81) and (83) provide for two unstable modes at large values of real $\hat{k}$ for the distribution in Fig 4(c).]

A nonexclusive example of a distribution for which neither $s_{1}$ nor $s_{2}$ vanishes (unless the parameter $a=\pi$ or $2 \pi$ ) is presented in Fig. 3(d). From Eq. (68), it follows that in this example $s_{1}=(2 / a) \exp (-i a / 2) \sin (a / 2)$ and $s_{2}=(1 / a) \exp (-i a) \sin a$. If both $s_{1}$ and $s_{2}$ are nonvanishing, determining the large $|\hat{k}|$ behavior of the dispersion relation in Eq. (69) is more difficult than in the previous cases. Four of the large- $|\hat{k}|$ branches are given by Eqs. (79) and (80). The behaviors of the remaining six branches (including all that may show growth at large, real $\hat{k})$ are determined by solving a cubic equation in $\left(\hat{\omega}-\hat{k} \beta_{z}-1\right)^{2}$. Further details will not be given in this paper. 
Unless $s_{1}=s_{2}=0$, some of the eigenmodes $\mathbf{E}$ in Eq. (58) will involve two or more of the components $E_{-}(\hat{k}, \hat{\omega}), E_{+}(\hat{k}, \hat{\omega}-2)$, and $E_{z}(\hat{k}, \hat{\omega}-1)$. A quantity that will be employed to measure the relative importance of $E_{-}(\hat{k}, \hat{\omega})$ and $E_{+}(\hat{k}, \hat{\omega}-2)$ is the Poynting flux ratio defined by

$$
\left|\frac{S_{+}(\hat{k}, \hat{\omega}-2)}{S_{-}(\hat{k}, \hat{\omega})}\right|=\left|\frac{\hat{\omega}}{\hat{\omega}-2}\right|\left|\frac{E_{1+}(\hat{k}, \hat{\omega}-2)}{E_{1-}(\hat{k}, \hat{\omega})}\right|^{2} .
$$

This quantity is the ratio of the time-averaged Poynting vectors that the field associated with each component would produce in the absence of the other component. A timedependent interference term due to the different frequencies of the components is not included.

\section{Numerical Examples.}

In the following numerical examples, $\hat{k}$ is restricted to be real. Then, $\operatorname{Im} \hat{\omega}>0$ indicates an unstable mode. If $\hat{k}$ is restricted to be real, then the transformation in Eq. (73) is equivalent to inverting a plot of Re $\hat{\omega}$ vs. $\hat{k}$ (real) through the point $(\hat{k}, \operatorname{Re} \hat{\omega})=(0,1)$ and reflecting a plot of Im $\hat{\omega}$ vs. $\hat{k}$ (real) through the Im $\hat{\omega}$-axis. It follows from the invariance of the dispersion relation in Eq. (69) under this transformation and from Eqs. (74)-(75) that there is no loss of generality if numerical examples are limited to the case of nonnegative real $\hat{k}$.

Parameter values in all of the numerical examples below in Figs. 4-7 are $\hat{\omega}_{p}^{2} / \hat{\omega}_{c}^{2}=0.05$, $\gamma_{0}=2$, and $\alpha_{0}=0.4$. In order to ensure that values selected for $s_{1}$ and $s_{2}$ are realistic (i.e., correspond to $\Phi(\xi) \geq 0$ in Eq. (65)), we assume that $\Phi(\xi)$ has one of the functional forms shown in Figs. 3(a)-3(d).

Example 1: If the distribution $\Phi(\xi)$ is uniform [see Fig. 3(a)], then $s_{1}=s_{2}=0$. [Such a distribution is also attained with $a=\pi$ in Fig. 3(b), $K=0$ in Fig. 3(c), or $a=2 \pi$ in Fig. 3(d).] In this case, the dispersion relation in Eq. (69) decouples into the three independent relations $M_{--}(\hat{k}, \hat{\omega})=0, M_{++}(\hat{k}, \hat{\omega})=0$, and $M_{z z}(\hat{k}, \hat{\omega})=$ 0 . These are respectively the dispersion relations for uncoupled right- and left-handed circularly polarized electromagnetic waves and for the electrostatic wave. For a given $\hat{k}$, the frequencies of these waves are $\hat{\omega}, \hat{\omega}-2$, and $\hat{\omega}-1$, respectively. Growth-rate curves 
(Im $\hat{\omega}$ vs. $\hat{k}$ ) for this limiting case are presented in Fig. 4(a) over the interval $0 \leq \hat{k} \leq 10$. Corresponding plots of Re $\hat{\omega}$ vs. $\hat{k}$ over the interval $0 \leq \hat{k} \leq 1.4$ are presented in Fig. 4(b). Letters on these plots designate corresponding points on the two diagrams. The points $B$ and $C$ in Fig. 4(a) coincide. The growth-rate curve segments $C D G$ and $H I$ are obtained from $M_{--}(\hat{k}, \hat{\omega})=0$, and a corresponding eigenmode $\mathbf{E}$ in Eq. (58) has only $E_{-}(\hat{k}, \hat{\omega})$ as a nonvanishing component. The growth-rate curve segments $A B$ and $F E J$ are obtained from $M_{++}(\hat{k}, \hat{\omega})=0$. A corresponding eigenmode has only one nonvanishing component, namely $E_{+}(\hat{k}, \hat{\omega}-2)$.

The plot of Rêे vs. $\hat{k}$ in Fig. 4(b) is needed for the proper interpretation of the growth-rate curves in Fig. 4(a). If $\hat{k}>0$ and Re $\hat{\omega}>0$, then $E_{1-}(\hat{k}, \hat{\omega})$ and $E_{1+}(\hat{k}, \hat{\omega})$ are respectively components associated with RHP and LHP waves that travel in the forward (positive-z) direction. If $\hat{k}>0$ (as before) but Re $\hat{\omega}<0$, then the handedness of these waves is unchanged, however they now travel in the backward (negative-z) direction. Similarily, the electrostatic wave associated with $E_{z}(\hat{k}, \hat{\omega})$ is backward traveling if $\hat{k}>0$ and $\operatorname{Re} \hat{\omega}<0$. As an illustration of the use of Fig. 4(b) in interpreting Fig. 4(a), consider the segmented growth-rate curve $A(B C) D G$, which gives the growth rate of the cyclotron maser instability. Segment $A B$ pertains to $E_{+}(\hat{k}, \hat{\omega}-2)$. From Fig. $4(\mathrm{~b})$, it is seen that $\operatorname{Re} \hat{\omega}-2<0$ everywhere on $A B$. Consequently, the growth-rate curve $A B$ in Fig. 4(a) pertains to growing, backward-traveling, LHP electromagnetic waves. Similarily, segment $C D G$ in Fig. 4(a) pertains to $E_{-}(\hat{k}, \hat{\omega})$. Reference to Fig. 4 (b) shows that Re $\hat{\omega}>0$ everywhere on $C D G$, so that growth-rate curve segment $C D G$ in Fig. 4(a) pertains to growing, forward-traveling, RHP electromagnetic waves. Similar analysis shows that the growth-rate curve $H I$ for the whistler instability pertains to forward-traveling, RHP electromagnetic waves. Also, the growth-rate curve segment $F E$ pertains to backward-traveling LHP waves, and the segment $E J$ pertains to forward-traveling LHP waves. Because all of the roots of $M_{z z}(\hat{k}, \hat{\omega})$ are real, no corresponding growth-rate curves appear in Fig. 4(a).

Electromagnetic and beam waves are said to be in resonance for the cyclotron maser instability when $\hat{\omega}=\hat{k}$ and $\hat{\omega}=\hat{k} \beta_{z}+1$. These resonance values of $\hat{\omega}$ and $\hat{k}$ are given by $\hat{\omega}_{r}=\hat{k}_{r}=1 /\left(1-\beta_{z}\right)$. In this example, $\hat{k}_{r}=4.94$. Fig. 4(a) conforms with the well-known fact that no growth of RHP radiation occurs at $\hat{k}=\hat{k}_{r}$ in an uncoupled system. 
Finally, it is emphasized that no special relation exists between the LHP and RHP waves considered above when $s_{1}=s_{2}=0$. However, the plots in Fig. 4 will be approached by any system using our parameters in the limit in which both $s_{1}$ and $s_{2}$ approach zero.

Example 2: An explicit example of a nonuniform distribution in phase is obtained by selecting $a=\pi / 4$ in Fig. 3(b). Then the unperturbed electron beam consists of two streams with respective distributions centered about $\xi=0$ and $\xi=\pi$. Each distribution is a water bag of width $\pi / 4$. The corresponding parameters defined in Eq. (68) are $s_{1}=0$ and $s_{2}=2 \sqrt{2} / \pi$. Because $s_{1}=0$, the eigenmodes $\mathbf{E}$ in Eq. (58) are of two types. The first type of eigenmode has only $E_{1 z}(\hat{k}, \hat{\omega})$ as a nonvanishing component. The corresponding dispersion relation is $M_{z z}(\hat{k}, \hat{\omega})=0$, which does not allow for growth. [See Eq. (76).] The second type of eigenmode has two nonvanishing components, namely $E_{1-}(\hat{k}, \hat{\omega})$ and $E_{1+}(\hat{k}, \hat{\omega}-2)$. Some of these eigenmodes are unstable.

Growth-rate curves for this system for $0 \leq \hat{k} \leq 15$ and corresponding plots of Re $\hat{\omega}$ vs. $\hat{k}$ (for $0 \leq \hat{k} \leq 1.5$ ) are presented in Figs. $5(\mathrm{a})$ and $5(\mathrm{~b})$. As a measure of the relative importance of the RHS and LHS electromagnetic waves associated with unstable modes, plots of the Poynting flux ratio in Eq. (85) as a function of $\hat{k}$ (for unstable modes only) are presented in Fig. 5(c). Letters show corresponding points on Figs. 5(a)-5(c). By comparing Figs. 5(a) and 5(b), we see that the growth-rate curve segments $B A, C D$, and $F E$ pertain to modes consisting of a forward-traveling RHP electromagnetic wave (because Rêे $>0$ ) and a backward-traveling LHP electromagnetic wave (because Rê $-2<0$ ). All other segments of the growth-rate curves pertain to modes consisting of forward traveling RHP and LHP electromagnetic waves.

In the case of a uniform distribution in $\xi$ (Example 1), no growth of RHP electromagnetic waves occurs at the resonance wavenumber $\hat{k}=\hat{k}_{r}=4.94$. [See Fig. 4(a).] The growth-rate curve $C D H$ in Fig. 5(a) shows a mode at $\hat{k}=\hat{k}_{r}=4.94$ which grows significantly faster than any mode in Fig. 4(a). From Fig. 5(a), we see that the RHP Poynting flux associated with the mode is almost twenty times the LHP Poynting flux. Consequently, growth of RHP radiation is now possible at $\hat{k}=\hat{k}_{r}$ although it must be accompanied by a smaller growing component of LHP radiation. We remark that among computations so far carried out those for systems with two-stream distributions in $\xi$ (with 
a phase difference of $\pi$ ) show the most rapid growth rates at $\hat{k}=\hat{k}_{r}$.

Referring to Fig. 5(a), we see that there are two unstable branches at large $\hat{k}$ in conformity with Eqs. (77)-(78). From Fig. 5(c), it is seen that, for either branch at large $\hat{k}$, the RHP Poynting flux is approximately double that of the LHP Poynting flux. Finally, note from Fig. 5(c) that for unstable eigenmodes at small $\hat{k}$ the ratio of the backward traveling LHP Poynting flux to the forward traveling RHP Poynting flux depends very strongly on the branch of the dispersion relation and varies rapidly with $\hat{k}$ for a given branch.

Example 3: As a second example of a nonuniform phase distribution, select the form of $\Phi(\xi)$ in Fig. 3(c) and choose the parameter value $K=1 / 2 \pi$. [Equivalently, we could let $a=\pi$ in Fig. 3(d).] Then the equilibrium particle phases are uniformly distributed between $\xi=0$ and $\xi=\pi$, and no particles have phases in the range $\pi<\xi<2 \pi$. Fourier components in Eq. (68) are $s_{1}=-2 i / \pi$ and $s_{2}=0$. Because $s_{2}=0$, the field components $E_{1-}(\hat{k}, \hat{\omega})$, and $E_{1+}(\hat{k}, \hat{\omega}-2)$ are indirectly coupled through the electrostatic component $E_{1 z}(\hat{k}, \hat{\omega}-1)$. Consequently, the eigenmodes $\mathbf{E}$ in Eq. (58) will (in general) have three nonvanishing field components. Growth-rate curves for this example are presented in Fig. $6(\mathrm{a})$ for $0 \leq \hat{k} \leq 10$, and corresponding plots of Rêे vs. $\hat{k}$ (for the interval $0 \leq \hat{k} \leq 2$ ) are given in Fig. 6(b). Plots of the Poynting flux ratio in Eq. (85) are shown in Fig. 6(c). Finally, as a measure of the relative importance of the electrostatic component of the unstable eigenmodes, we present a plot of $2^{-1 / 2}\left|E_{1-}(\hat{k}, \hat{\omega}) / E_{1 z}(\hat{k}, \hat{\omega}-1)\right|$ vs. $\hat{k}$ in Fig. $6(\mathrm{~d})$. (The factor of $2^{-1 / 2}$ appears in the field ratio because $2^{-1 / 2} E_{ \pm}$is the proper normalization of coefficients of the complex unit vectors for LHP and RHP waves when comparison is to be made with Cartesian field components.) Letters show corresponding points in Figs. 6(a)-6(d).

Reference to Figs. 6(a) and 6(b) shows that the wave associated with the component $E_{1+}(\hat{k}, \hat{\omega}-2)$ is left-hand polarized and backwards traveling for eigenmodes on the growth-rate curve segments $G H, A B C$, and $D E F$. The electrostatic wave associated with $E_{1 z}(\hat{k}, \hat{\omega}-1)$ is forward traveling for all unstable modes except for those modes on the growth-rate curve $A B C$ for which $\hat{k}$ is very close to zero. All other components of unstable eigenmodes represent forward-traveling waves.

Referring to Fig. 6(a), we see that two unstable branches of the dispersion relation 
are present at large values of $\hat{k}$ in conformity with Eq. (81) and (83). From Figs. 6(c) and 6(d), it is seen that the RHP Poynting flux exceeds that of the LHP electromagnetic wave by a factor of approximately three for both branches at large $\hat{k}$. The electrostatic contribution to eigenmodes on the upper branch is relatively very small. On the other hand, the electrostatic field amplitude in eigenmodes on the lower branch is of the same order of magnitude as the LHP electromagnetic field amplitude.

Two branches, $M N O$ and $G H I$, show moderate growth rates at the resonance $\hat{k}=\hat{k}_{r}=4.94$. Reference to Figs. 6(c) and 6(d) shows that the RHP Poynting flux is significantly larger than the LHP Poynting flux for the eigenmodes associated with either of these branches at $\hat{k}=\hat{k}_{r}$. Moreover, $\left|E_{1-}(\hat{k}, \hat{\omega}) / E_{1 z}(\hat{k}, \hat{\omega}-1)\right|>10$ for either branch at $\hat{k}=\hat{k}_{r}$. Again, this behavior is in contrast with the case of a uniform distribution in $\xi$ where no growth of RHP electromagnetic radiation takes place at the resonance value of $\hat{k}$. However, the growth-rates at $\hat{k}=\hat{k}_{r}$ in Fig. 6(a) are not large, being slightly less than the maximum growth-rates that appear in Fig. 4(a) for the case of a uniform distribution.

Although the growth-rate peak $A B C$ is very narrow, eigenmodes at points near its maximum are the fastest growing modes of this system. Moreover, reference to Fig. 6(c) shows that these modes contain a relatively strong backward-traveling, LHP component.

Example 4: As our final numerical example, we treat the limit of $a=0$ for the distribution in Fig. 3(d). In the limit, the distribution becomes $\Phi(\xi)=\sum_{-\infty}^{\infty} \delta(\xi-2 n \pi)$ with $s_{1}=s_{2}=1$. In this case, $\phi=\omega_{c} t$ for all particles in the equilibrium beam. Growth-rate curves for the interval $0 \leq \hat{k} \leq 8$ and plots of Re $\hat{\omega}$ vs. $\hat{k}$ for the interval $0 \leq \hat{k} \leq 1.5$ appear in Figs. 7(a) and 7(b), respectively. For unstable eigenmodes, plots of the Poynting flux ratio in Eq. (85) vs. $\hat{k}$ and $2^{-1 / 2}\left|E_{1-}(\hat{k}, \hat{\omega}) / E_{1 z}(\hat{k}, \hat{\omega}-1)\right|$ vs. $\hat{k}$ are presented in Figs. $7(\mathbf{c})$ and $7(\mathrm{~d})$, respectively. Letters on these graphs show corresponding points. Eigenmodes belonging to growth-rate curve segments $A B C$ and $D E$ have backward-traveling LHP components and forward-traveling RHP and electrostatic components. A tiny growth-rate peak appears at $G$ in Fig. 7(a). Reference to Fig. 7(b) shows that its LHP and electrostatic components are backward traveling. Eigenmodes on all other segments contain only forward-traveling components.

A striking feature of the growth-rate curves in Fig. $7(a)$ is that no growth occurs 
for values of $\hat{k}$ greater than approximately 6.2. That is, no branches of the dispersion relation show growth in the limit of large $\hat{k}$. [Suppression of instability in the whistler by the electrostatic wave is discussed in Ref. 9.] Also, notice the interval of no growth $F H$ $(1.4 \lesssim \hat{k} \lesssim 2)$.

Another striking feature is the great height of the growth peak $A B C$. From Fig. 7(c), we see that the Poynting flux of the backward-traveling LHP electromagnetic wave exceeds that of the forward-traveling RHP electromagnetic wave over most of the interval of this growth peak. The amplitude of the forward-traveling electrostatic wave is seen [from Figs. 7(c) and 7(d)] to be of the same order of magnitude as the amplitudes of the electromagnetic waves.

Again, we see growth of RHP electromagnetic waves at the resonance wavenumber $\hat{k}=\hat{k}_{r}=4.94$. In fact, the maximum of the growth-rate curve HIJ in Fig. 7(a) is situated very close to the resonance wavenumber, and the growth rate at this maximum exceeds any growth rate for the gyrotropic case is Fig. 4(a). From Figs. 7(c) and 7(d), it is seen that the largest component for eigenmodes near this maximum is that corresponding to forward-traveling RHP electromagnetic radiation.

To summarize, it is evident that a richness of structure in the growth-rate curves can be produced by introducing nonuniform distributions $\Phi(\xi)$. Using proper choices of $\Phi(\xi)$, temporal growth rates near $k=\hat{k}_{r}$ can be significantly increased and growth rates at large $\hat{k}$ can be on the one hand enhanced or on the other hand completely suppressed. Two-stream equilibrium distributions such as that in Example 2 seem to be most effective in enhancing growth rates at the resonance $\hat{k}=\hat{k}_{r}$. Gaps of no growth can be introduced at moderate values of $\hat{k}$. At small values of $\hat{k}$ where eigenmodes may contain backward-traveling components, growth rates and the properties of eigenmodes can be greatly changed by changing $\Phi(\xi)$. [This latter fact suggests, but does not prove, that absolute instability properties may depend strongly on $\Phi(\xi)$. However, no pinch-point analyses of these systems have been carried out. ${ }^{26-28}$ ] 
IV. Dispersion Characteristics for the Axial-Dependent Equilibrium Distribution without Axial Momentum Spread.

\section{A. Analysis}

Finally, we consider the axial-dependent equilibrium distribution $f_{0}\left(p_{\perp}, p_{z}, \zeta\right)=$ $f_{0}\left(p_{\perp}, p_{z}, \phi-m \Omega_{c} z / p_{z}\right)$ for which the perturbed field components are related by the integral equations (41)-(43). Even if the equilibrium distribution is not uniformly random in $\phi$, Eqs. (41)-(43) will reduce to algebraic equations if there is no spread in $p_{z}$ in the equilibrium distribution. (A spread in energy is permitted if it is due only to a spread in $p_{\perp}$.) The most general equilibrium distribution having this property is

$$
f_{0}\left(p_{\perp}, p_{z}, \zeta\right)=\delta\left(p_{z}-p_{z 0}\right) \hat{f}_{0}\left(p_{\perp}, p_{z 0}, \zeta\right)
$$

It follows from Eq. (40) that

$$
h_{n}\left(p_{\perp}, p_{z}\right)=\delta\left(p_{z}-p_{z 0}\right) \hat{h}_{n}\left(p_{\perp}, p_{z 0}\right)
$$

where

$$
\hat{h}_{n}\left(p_{\perp}, p_{z 0}\right)=\frac{1}{\sqrt{2 \pi}} \int_{0}^{2 \pi} d \zeta \hat{f}_{0}\left(p_{\perp}, p_{z 0}, \zeta\right) \exp (-i n \zeta) .
$$

Three homogeneous equations relating just three field components are found using a procedure similar to that used in obtaining Eq. (58). In matrix form the equations are

$$
\mathrm{D}^{\prime} \mathrm{E}^{\prime}=0
$$

where

$$
\mathbf{D}^{\prime}=\left(\begin{array}{ccc}
\hat{D}_{--}^{\prime}\left(k, \omega, \Omega_{c}, p_{z 0}\right) & -\hat{\eta}_{-+}\left(k, \omega, \Omega_{c}, p_{z 0}\right) & -\hat{\eta}_{-z}\left(k, \omega, \Omega_{c}, p_{z 0}\right) \\
-\hat{\eta}_{+-}\left(k+\frac{2 m \Omega_{c}}{p_{z 0}}, \omega, \Omega_{c}, p_{z 0}\right) & \hat{D}_{++}^{\prime}\left(k+\frac{2 m \Omega_{c}}{p_{z 0}}, \omega, \Omega_{c}, p_{z 0}\right) & -\hat{\eta}_{+z}\left(k+\frac{2 m \Omega_{c}}{p_{z 0}}, \omega, \Omega_{c}, p_{z 0}\right) \\
-\hat{\eta}_{z-}\left(k+\frac{m \Omega_{c}}{p_{z 0}}, \omega, \Omega_{c}, p_{z 0}\right) & -\hat{\eta}_{z+}\left(k+\frac{m \Omega_{c}}{p_{z 0}}, \omega, \Omega_{c}, p_{z 0}\right) & \hat{D}_{z z}^{\prime}\left(k+\frac{m \Omega_{c}}{p_{z 0}}, \omega, p_{0}\right)
\end{array}\right)
$$

and

$$
\mathbf{E}^{\prime}=\left(\begin{array}{c}
E_{1-}(k, \omega) \\
E_{1+}\left(k+\frac{2 m \Omega_{c}}{p_{z 0}}, \omega\right) \\
E_{1 z}\left(k+\frac{m \Omega_{c}}{p_{z 0}}, \omega\right)
\end{array}\right)
$$


The quantities $\hat{\eta}_{i j}$ in Eq. (90) are obtained from the corresponding quantities in Eqs. (47)(52) by replacing $h_{n}\left(p_{\perp}, p_{z}\right)$ with $\left.\hat{h}_{n}\left(p_{\perp}, p_{z}\right)\right), p_{z}$ with $p_{z 0}$, and $\gamma$ with $\gamma\left(p_{\perp}, p_{z 0}\right)$. The remaining quantities in Eq. (90) can be obtained from Eqs. (44)-(46). They are

$$
\begin{aligned}
& \hat{D}_{--}^{\prime}\left(k, \omega, \Omega_{c}, p_{z 0}\right)=\omega^{2}-c^{2} k^{2}-\frac{\sqrt{2 \pi}}{2} \Omega_{p}^{2} \int_{0}^{\infty} d p_{\perp} \hat{h}_{0}\left(p_{\perp}, p_{z 0}\right) \frac{p_{\perp}}{\gamma\left(p_{\perp}, p_{z 0}\right)} \\
& \times\left[2\left(\omega-\frac{k p_{z 0}}{\gamma\left(p_{\perp}, p_{z 0}\right) m}\right)\left(\omega-\frac{k p_{z 0}}{\gamma\left(p_{\perp}, p_{z 0}\right) m}-\frac{\Omega_{c}}{\gamma\left(p_{\perp}, p_{z 0}\right)}\right)^{-1}\right. \\
&\left.-\frac{p_{\perp}^{2}}{\gamma^{2}\left(p_{\perp}, p_{z 0}\right) m^{2} c^{2}}\left(\omega^{2}-c^{2} k^{2}\right)\left(\omega-\frac{k p_{z 0}}{\gamma\left(p_{\perp}, p_{z 0}\right) m}-\frac{\Omega_{c}}{\gamma\left(p_{\perp}, p_{z 0}\right)}\right)^{-2}\right] \\
& \hat{D}_{++}^{\prime}\left(k, \omega, \Omega_{c}, p_{z 0}\right)= \hat{D}_{--}^{\prime}\left(k, \omega,-\Omega_{c}, p_{z 0}\right), \\
& \hat{D}_{z z}^{\prime}\left(k, \omega, p_{z 0}\right)=-\sqrt{2 \pi} \Omega_{p}^{2} \int_{0}^{\infty} d p_{\perp} p_{\perp} \hat{h}_{0}\left(p_{\perp}, p_{z 0}\right) \gamma^{-1}\left(p_{\perp}, p_{z 0}\right) \\
& \times\left(1-\frac{p_{z 0}^{2}}{\gamma^{2}\left(p_{\perp}, p_{z 0}\right) m^{2} c^{2}}\right)\left(\omega-\frac{k p_{z 0}}{\gamma\left(p_{\perp}, p_{z 0}\right) m}\right)^{-2} .
\end{aligned}
$$

Once the assumption is made that equilibrium self fields can be neglected, the exact dispersion relation for the case of definite $p_{z}=p_{z 0}$ is

$$
\operatorname{det} \mathrm{D}^{\prime}(k, \omega)=0
$$

For a given frequency $\omega$, the wavenumber of the right-hand polarized wave $E_{1-}$ is $k$. The wavenumbers of the fields $E_{1+}$ and $E_{1 z}$ are $k+2 m \Omega_{c} / p_{z 0}$ and $k+m \Omega_{c} / p_{z 0}$, respectively. This result shows why integral equations relate the field components if a spread in $p_{z}$ is present in $f_{0}\left(p_{\perp}, p_{z}, \phi-m \Omega_{c} z / p_{z}\right)$. Then, $E_{1-}(k, \omega)$ couples to $E_{1+}\left(k+2 m \Omega_{c} / p_{z}, \omega\right)$ and to $E_{1 z}\left(k+m \Omega_{c} / p_{z}, \omega\right)$ over a continuum of values of $m \Omega_{c} / p_{z}$.

Finally, we emphasize that the eigenmode E in Eq. (60) is of a different nature than the eigenmode $\mathbf{E}^{\prime}$ in Eq. (92). The eigenmode $\mathbf{E}$ is a composite of three components which refer to waves of the same propagation vector but of different frequencies. These frequencies differ by fixed real values. On the other hand, the eigenmode $\mathbf{E}^{\prime}$ is a composite of three modes which refer to waves of the same frequency but of different propagation vectors. These propagation vectors differ by fixed real values. In either case, if $\hat{k}$ is restricted to real values, then temporal growth or decay rates are given by $\operatorname{Im} \hat{\omega}$ for all components. If 
$\hat{\omega}$ is restricted to real values, then spatial growth or decay rates are given by $\operatorname{Im} \hat{k}$ for all components.

\section{B. Case of Definite $p_{\perp}$ and $p_{z}$}

As in Sec. III, we present numerical examples for cases in which both $p_{z}=p_{z 0}$ and $p_{\perp}=p_{\perp 0}$ have definite values in the equilibrium distribution. Consequently, the factor $\hat{f}_{0}\left(p_{\perp}, p_{z 0}, \zeta\right)$ in Eq. $(86)$ is selected to be of the form

$$
\hat{f}_{0}\left(p_{\perp}, p_{z 0}, \zeta\right)=\frac{\delta\left(p_{\perp}-p_{\perp 0}\right)}{p_{\perp}} \Psi(\zeta)
$$

where $\Psi(\zeta)$ is a periodic function of $\zeta$ (or $\phi$ ) of period $2 \pi$. From Eq. (5), the normalization condition on $\Psi(\zeta)$ is

$$
\int_{0}^{2 \pi} d \phi \Psi\left(\phi-m \frac{\Omega_{c}}{p_{z}} z\right)=\int_{0}^{2 \pi} d \zeta \Psi(\zeta)=1 .
$$

It follows from Eq. (88) that

$$
\hat{h}_{n}\left(p_{\perp}, p_{z 0}\right)=\frac{1}{\sqrt{2 \pi}} \frac{\delta\left(p_{\perp}-p_{\perp 0}\right)}{p_{\perp}} w_{n}
$$

where

$$
w_{n}=\int_{0}^{2 \pi} d \zeta \Psi(\zeta) \exp (-i n \zeta)
$$

Notice that $w_{0}=1$ and that $w_{-n}=w_{n}^{*}$.

With the aid of Eqs. (92)-(94) and Eqs. (46)-(52), we obtain the dispersion relation in Eq. (95) for the case of definite $p_{\perp}$ and $p_{z}$. The dispersion relation is

$$
\begin{aligned}
M_{--} & (\hat{k}, \hat{\omega}) M_{++}^{\prime}(\hat{k}, \hat{\omega}) M_{z z}(\hat{k}, \hat{\omega})= \\
& \frac{1}{2}\left(\frac{\omega_{p}^{2}}{\omega_{c}^{2}}\right)^{2} \beta_{\perp}^{2}\left|w_{1}\right|^{2}\left\{\left(\beta_{z} \hat{\omega}-\hat{k}\right)^{2} M_{++}^{\prime}(\hat{k}, \hat{\omega})+\left[\beta_{z} \hat{\omega}-\left(\hat{k}+\frac{2}{\beta_{z}}\right)\right]^{2} M_{--}(\hat{k}, \hat{\omega})\right\} \\
& +\frac{1}{4}\left(\frac{\omega_{p}^{2}}{\omega_{c}^{2}}\right)^{2} \beta_{\perp}^{4}\left|w_{2}\right|^{2}\left[\hat{\omega}^{2}-\hat{k}\left(\hat{k}+\frac{2}{\beta_{z}}\right)\right]^{2} M_{z z}(\hat{k}, \hat{\omega}) \\
& -\frac{1}{4}\left(\frac{\omega_{p}^{2}}{\omega_{c}^{2}}\right)^{3} \beta_{\perp}^{4}\left(w_{2} w_{-1}^{2}+w_{-2} w_{1}^{2}\right)\left[\hat{\omega}^{2}-\hat{k}\left(\hat{k}+\frac{2}{\beta_{z}}\right)\right]\left[\beta_{z} \hat{\omega}-\left(\hat{k}+\frac{2}{\beta_{z}}\right)\right]\left(\beta_{z} \hat{\omega}-\hat{k}\right) .
\end{aligned}
$$


Dimensionless frequencies, wavenumbers, and velocity components are defined as in Sec. III.B, and

$$
\begin{aligned}
& M_{++}^{\prime}(\hat{k}, \hat{\omega})=\left[\hat{\omega}^{2}-\left(\hat{k}+\frac{2}{\beta_{z}}\right)^{2}\right]\left(\hat{\omega}-\hat{k} \beta_{z}-1\right)^{2}-\frac{\omega_{p}^{2}}{\omega_{c}^{2}}\left(\hat{\omega}-\hat{k} \beta_{z}-2\right)\left(\hat{\omega}-\hat{k} \beta_{z}-1\right) \\
& \quad+\frac{1}{2} \frac{\omega_{p}^{2}}{\omega_{c}^{2}} \beta_{\perp}^{2}\left[\hat{\omega}^{2}-\left(\hat{k}+\frac{2}{\beta_{z}}\right)^{2}\right] .
\end{aligned}
$$

The quantities $M_{--}(\hat{k}, \hat{\omega})$ and $M_{z z}(\hat{k}, \hat{\omega})$ are defined in Eqs. (70) and (72), respectively.

Like Eq. (69), Eq. (100) is valid for complex $\hat{\omega}$ and complex $\hat{k}$. It is invariant under the transformation

$$
\begin{aligned}
& \hat{k} \longrightarrow-\hat{k}^{*}-\frac{2}{\beta_{z}}, \\
& \hat{\omega} \longrightarrow-\hat{\omega}^{*} .
\end{aligned}
$$

In analogy with Eq. (74), it follows from the matrix equation (90) that, if either $w_{1} \neq 0$ or $w_{2} \neq 0$, then under the transformation in Eq. (102)

$$
\frac{E_{1+}\left(\hat{k}+2 / \beta_{z}, \hat{\omega}\right)}{E_{1-}(\hat{k}, \hat{\omega})} \longrightarrow \frac{E_{1+}\left(-\hat{k}^{*},-\hat{\omega}^{*}\right)}{E_{1-}\left(-\hat{k}^{*}-2 / \beta_{z},-\hat{\omega}^{*}\right)}=\frac{E_{1-}^{*}(\hat{k}, \hat{\omega})}{E_{1+}^{*}\left(\hat{k}+2 / \beta_{z}, \hat{\omega}\right)},
$$

Moreover, in analogy with Eq. (75), if $w_{1} \neq 0$, then under the transformation in Eq. (102)

$$
\begin{aligned}
& \frac{E_{1+}\left(\hat{k}+2 / \beta_{z}, \hat{\omega}\right)}{E_{1 z}\left(\hat{k}+1 / \beta_{z}, \hat{\omega}\right)} \rightarrow \frac{E_{1+}\left(-\hat{k}^{*},-\hat{\omega}^{*}\right)}{E_{1 z}\left(-\hat{k}^{*}-1 / \beta_{z},-\hat{\omega}^{*}\right)}=\frac{E_{1-}^{*}(\hat{k}, \hat{\omega})}{E_{1 z}^{*}\left(\hat{k}+1 / \beta_{z}, \hat{\omega}\right)}, \\
& \frac{E_{1-}(\hat{k}, \hat{\omega})}{E_{1 z}\left(\hat{k}+1 / \beta_{z}, \hat{\omega}\right)} \longrightarrow \frac{E_{1-}\left(-\hat{k}^{*}-2 / \beta_{z},-\hat{\omega}^{*}\right)}{E_{1 z}\left(-\hat{k}^{*}-1 / \beta_{z},-\hat{\omega}^{*}\right)}=\frac{E_{1+}^{*}\left(\hat{k}+2 / \beta_{z}, \hat{\omega}\right)}{E_{1 z}^{*}\left(\hat{k}+1 / \beta_{z}, \hat{\omega}\right)} .
\end{aligned}
$$

The ratio of the time-averaged Poynting vectors associated with the individual $E_{1+}\left(\hat{k}+2 / \beta_{z}, \hat{\omega}\right)$ and $E_{1-}(\hat{k}, \hat{\omega})$ fields is

$$
\left|\frac{S_{+}\left(\hat{k}+2 / \beta_{z}, \hat{\omega}\right)}{S_{-}(\hat{k}, \hat{\omega})}\right|=\left|\frac{\hat{k}+2 / \beta_{z}}{\hat{k}}\right|\left|\frac{E_{1+}\left(\hat{k}+2 / \beta_{z}, \hat{\omega}\right)}{E_{1-}(\hat{k}, \hat{\omega})}\right|^{2} .
$$


Like the dispersion relation in Eq. (69) of Sec. III.B, Eq. (100) is a tenth-degree polynomial equation in either $\hat{k}$ or $\hat{\omega}$. The large $|\hat{k}|$ behavior of $\hat{\omega}(\hat{k}) / \hat{k}$ given by Eq. (100) is the same as that given by Eq. (69), including two branches with $\hat{\omega}(\hat{k}) / \hat{k} \simeq 1$, two branches with $\hat{\omega}(\hat{k}) / \hat{k} \simeq-1$, and six branches with $\hat{\omega}(\hat{k}) / \hat{k} \simeq \hat{k} \beta_{z}$.

If either $w_{1}$ or $w_{2}$ vanishes, approximations for $\hat{\omega}(\hat{k})$ valid for large values of $\hat{k}$ are readily determined and are found to be very similar to those found in the previous section for the case of the time-dependent equilibrium. If $w_{1}=0$ and $w_{2} \neq 0$, then the transverse electromagnetic components are coupled to each other and the electrostatic component is uncoupled. The large $|\hat{k}|$ behaviors of the ten branches of the dispersion relation in Eq. (100) are similar to those given in Eqs. (76)-(80) for the corresponding case $\left(s_{1}=\right.$ $0, s_{2} \neq 0$ ) of the dispersion relation in Eq. (69). Four of the branches now obey Eqs. (76) and (79). Four branches obey Eqs. (77) and (78) with $s_{2}$ replaced by $w_{2}$, that is

$$
\begin{aligned}
& \hat{\omega} \simeq \hat{k} \beta_{z}+1 \pm \frac{i}{\sqrt{2}} \frac{\omega_{p}}{\omega_{c}} \beta_{\perp}\left(1+\left|w_{2}\right|\right)^{1 / 2}, \\
& \hat{\omega} \simeq \hat{k} \beta_{z}+1 \pm \frac{i}{\sqrt{2}} \frac{\omega_{p}}{\omega_{c}} \beta_{\perp}\left(1-\left|w_{2}\right|\right)^{1 / 2} .
\end{aligned}
$$

Finally, Eq. (82) is no longer valid and is replaced by

$$
\hat{\omega} \simeq \pm\left[\left(\hat{k}+2 \beta_{z}\right)^{2}+\frac{\omega_{p}^{2}}{\omega_{c}^{2}}\right]^{1 / 2},
$$

for the two remaining branches. Growth-rate curves for $\left|w_{2}\right|<1$ will show two unstable branches of the dispersion relation in Eq. (100) for large values of real $\hat{k}$.

If $w_{1} \neq 0$ and $w_{2}=0$, then the transverse electromagnetic components of an eigenmode are coupled through the longitudinal electrostatic component. The large $|\hat{k}|$ behavior of the dispersion relation in Eq. (100) is similar to that of the dispersion relation in Eq. (69) for the analogous case of $s_{1} \neq 0$ and $s_{2}=0$. Four of the branches obey Eqs. (79) and (81). Four additional branches obey Eqs. (82) and (83) with the quantity $s_{1}$ in Eq. (84) replaced with $w_{1}$, that is

$$
\begin{aligned}
& \hat{\omega} \simeq \hat{k} \beta_{z}+1 \pm \frac{1}{\sqrt{2}} \frac{\omega_{p}}{\omega_{c}} \kappa_{+}^{\prime}, \\
& \hat{\omega} \simeq \hat{k} \beta_{z}+1 \pm \frac{i}{\sqrt{2}} \frac{\omega_{p}}{\omega_{c}} \kappa_{-}^{\prime},
\end{aligned}
$$


where

$$
\kappa_{ \pm}^{\prime}=\left\{\mp\left(\frac{\beta_{\perp}^{2}}{2}-\left(1-\beta_{z}^{2}\right)\right)+\left[\left(\frac{\beta_{\perp}^{2}}{2}-\left(1-\beta_{z}^{2}\right)\right)^{2}+4 \beta_{\perp}^{2}\left(1-\beta_{z}^{2}\right)\left(\frac{1}{2}-\left|w_{1}\right|^{2}\right)\right]^{\frac{1}{2}}\right\}^{\frac{1}{2}}
$$

The remaining two branches obey Eq. (106). At large real $\hat{k}$, growth-rate curves will show two unstable branches of the dispersion relation in Eq. (100) if $\left|w_{1}\right|<1 / \sqrt{2}$.

Finally, if neither $w_{1}$ nor $w_{2}$ vanish, then the large $|\hat{k}|$ behaviors of four of the branches of the dispersion relation in Eq. (100) are given by Eqs. (79) and (106). The behaviors of the remaining six branches are obtained by solving a cubic equation in $\left(\hat{\omega}-\hat{k} \beta_{z}-1\right)^{2}$. We do not include an analysis of the equation in this paper.

\section{Numerical Examples}

Before the numerical examples are presented, one final point of clarification must be made. Throughout this paper, we have followed the usual terminology and referred to $E_{1-}(\hat{k}, \hat{\omega})$ and $E_{1+}(\hat{k}, \hat{\omega})$ as components representing RHP- and LHP-electromagnetic waves, respectively. However, this nomenclature is proper only if $\operatorname{Re} \hat{k}>0$. If $\operatorname{Re} \hat{k}<0$, then the roles played by these components are reversed and $E_{1-}(\hat{k}, \hat{\omega})$ and $E_{1+}(\hat{k}, \hat{\omega})$ represent LHP and RHP electromagnetic waves, respectively. If (in addition) Re $\hat{\omega}>0$, then both waves are backward traveling. Moreover, if $\operatorname{Re} \hat{\omega}<0$, then both waves are forward traveling.

As in Sec. III, the following numerical computations are limited to the case of real $\hat{k}$. Then, from Eq. (102), Eq. (100) is invariant under the transformation $\hat{\omega} \rightarrow-\hat{\omega}^{*}$ and $\hat{k} \rightarrow-\hat{k}-2 / \beta_{z}$. This transformation is equivalent to inverting a plot of $\operatorname{Re} \hat{\omega}$ vs. $\hat{k}$ (real) through the point $(\hat{k}, \operatorname{Re} \hat{\omega})=\left(-1 / \beta_{z}, 0\right)$ and reflecting a plot of $\operatorname{Im}(\hat{\omega})$ vs. $\hat{k}$ (real) through the vertical line $\hat{k}=-1 / \beta_{z}$. Because of Eqs. (103) and (104), no new information is obtained from the transformed eigenvectors. Consequently, the region $\hat{k}<-1 / \beta_{z}$ is omitted from the following plots.

Parameter values used below are the same as those used in previous numerical examples (i.e., $\hat{\omega}_{p}^{2} / \hat{\omega}_{c}^{2}=0.05, \gamma_{0}=2$, and $\alpha_{0}=0.4$ ). Functional forms considered for $\Psi(\zeta)$ are chosen as $\Psi(\xi)=\Phi(\xi)$, where $\Phi(\xi)$ is defined in Figs. 3(a)-3(d). Moreover each of the examples below is the analogue (for the axial-dependent distribution) of the example of the same number in Sec. III.C (for the time-dependent distribution). 
Example 1: For $w_{1}=w_{2}=0$, which corresponds to $\Psi(\xi)=\Phi(\xi)$ shown in Fig. 3(a), the dispersion relation in Eq. (100) reduces to the three independent dispersion relations $M_{--}(\hat{k}, \hat{\omega})=0, M_{++}^{\prime}(\hat{k}, \hat{\omega})=0$, and $M_{z z}(\hat{k}, \hat{\omega})=0$ for uncoupled right- and leftcircularly polarized transverse waves and the longitudinal electrostatic wave, respectively. For a given frequency, the respective wavenumbers for these waves are $\hat{k}, \hat{k}+2 / \beta_{z}$, and $\hat{k}+1 / \beta_{z}$. Growth-rate curves are shown in Fig. 8(a) for $-1 / \beta_{z}=-1.254 \leq \hat{k} \leq 10$. Plots of Re $\hat{\omega}$ vs. $\hat{k}$ for $-1 / \beta_{z} \leq \hat{k} \leq 0$ are presented in Fig. $8(\mathrm{~b})$. Letters show corresponding points in Figs. 8(a) and 8(b).

In Fig. 8(a), the growth-rate curve segments $G H$ and $H I$ are obtained from roots of $M_{++}^{\prime}(\hat{k}, \hat{\omega})$, so that the corresponding eigenmodes have a single nonvanishing component $E_{1+}\left(\hat{k}+2 / \beta_{z}, \hat{\omega}\right)$. Referring to Fig. $8(\mathrm{~b})$, we see that Re $\hat{\omega}>0$ and $\hat{k}+2 / \beta_{z}>0$ on both segments. Consequently, both segments represent unstable electromagnetic waves that are LHP and forward traveling. All other growth-rate curve segments in Fig. 8(a) are obtained from roots of $M_{--}(\hat{k}, \hat{\omega})=0$, so that the corresponding eigenmodes have a single nonvanishing component $E_{1-}(\hat{k}, \hat{\omega})$. For all points of the short growth-rate curve segment $A B$, reference to Fig. 8 (b) shows that $\hat{k}<0$ and Re $\hat{\omega}<0$. Consequently, growthrate curve segment $A B$ pertains to unstable, forward-traveling LHP electromagnetic waves. Similarily, $\hat{k}<0$ and Re $\hat{\omega}>0$ for eigenmodes on growth-rate curve segments $B C$ and $D E$, so that these segments represent unstable backward-traveling, LHP electromagnetic waves. The remaining growth-rate curve segments ( $E F$ and $J K$ ) pertain to unstable forward-traveling, RHP electromagnetic waves. As expected, there is no growth of the RHP electromagnetic wave at the resonance wavenumber $k_{r}=1 /\left(1-\beta_{z}\right)=4.94$. The eigenmodes obtained from roots of $M_{++}^{\prime}(\hat{k}, \hat{\omega})$ are of course completely decoupled from the eigenmodes obtained from roots of $M_{--}(\hat{k}, \hat{\omega})=0$. Nevertheless, Fig. 8(a) represents the limit approached by any system with our parameters as both $w_{1}$ and $w_{2}$ approach zero.

This example is analogous to Example 1 shown in Figs. 4(a) and 4(b) in Sec. III.C. Comparing Figs. 4(a) and 8(a), we see that they differ in two respects. First, the growthrate curve in Fig. 8(a) obtained from $M_{++}^{\prime}(\hat{k}, \hat{\omega})=0$ has the same form as the growthrate curve in Fig. $4(\mathrm{a})$ obtained from $M_{++}(\hat{k}, \hat{\omega})=0$ but is displaced to the left by $2 / \beta_{z}=2.508$. Second, no information is lost in Figs. 4(a) and 4(b) by ignoring the 
negative $\hat{k}$-axis. However, only $\hat{k}<-1 / \beta_{z}$ can be ignored in Figs. $8(\mathrm{a})$ and $8(\mathrm{~b})$ without losing information.

Example 2: For $w_{1}=0$ and $w_{2}=2 \sqrt{2} / \pi$, which can be obtained for $\Psi(\xi)=\Phi(\xi)$ in Fig. 3(b) with $a=\pi / 4$, the equilibrium beam consists of two streams, each with a water-bag distribution in $\phi$ of width $\pi / 4$. One distribution is centered at $\phi=m \Omega_{c} z / p_{z 0}$ and the other at $\phi=m \Omega_{c} z / p_{z 0}+\pi$. As $z$ varies, each center rotates about the direction of the applied field lines with a characteristic wavelength of $2 \pi p_{z 0} / m \Omega_{c}=2 \pi v_{z 0} / \omega_{c}$.

Growth-rate curves (for $-1.25 \leq \hat{k} \leq 16$ ) and corresponding plots of Re $\hat{\omega}$ vs. $\hat{k}$ (for $-1.25 \leq \hat{k} \leq 0)$ are presented in Figs. 9(a) and 9(b). Plots of the Poynting ratio in Eq. (105) vs. $\hat{k}$ (for $-1.25 \leq \hat{k} \leq 16$ ) appear in Fig. 9(c). Letters show corresponding points on these plots. The letters have also been chosen to correlate with letters on the corresponding plots for Example 2 of Sec. III.C in Figs. 5(a)-5(c), which is analogous to the present example. Superficially the plots in Figs. 9(a)-9(c) are very similar to the corresponding plots in Figs. 5(a)-5(c). However, it is emphasized that the eigenmodes are very different in the two cases. The eigenmodes for Fig. 5 consist of the nonvanishing components $E_{1-}(\hat{k}, \hat{\omega})$ and $E_{1+}(\hat{k}, \hat{\omega}-2)$, whereas the eigenmodes for Fig. 9 consist of the nonvanishing components $E_{1-}(\hat{k}, \hat{\omega})$ and $E_{1+}\left(\hat{k}+2 / \beta_{z}, \hat{\omega}\right)$.

By comparing Figs. 9(a) and 9(b), it is easily seen that the eigenmodes belonging to the growth-rate curve segments $B A, C D$, and $F E$ consist of LHP, forward-traveling electromagnetic waves [from $\left.E_{1+}\left(\hat{k}+2 / \beta_{z}, \hat{\omega}\right)\right]$ and LHP, backward-traveling electromagnetic waves [from $\left.E_{1-}(\hat{k}, \hat{\omega})\right]$. [The corresponding modes in Fig. 5 consist of RHP, forwardtraveling and LHP, backward-traveling electromagnetic waves.] From Fig. 9(c), we see that the backward Poynting flux is relatively strong for most eigenmodes on $C A$ and that it varies rapidly with $\hat{k}$ for eigenmodes on $C D$ and $F E$. The infinity in the Poynting flux ratio at the cutoff at $\hat{k}=0$ is due to the factor $\left|\left(\hat{k}+2 \beta_{z}\right) / \hat{k}\right|$ in Eq. (105). All of the remaining growth-rate curve segments in Fig. 9(a) pertain to eigenmodes consisting of a forward-traveling RHP and a forward-traveling LHP component. Notice that the branch $C D H$ of the dispersion relation shows a growth rate at the resonance $\hat{k}_{r}=4.94$ which is significantly greater than any growth rate for the uncoupled system in Fig. 8(a). Reference to Fig. 9(c) shows that the Poynting flux of RHP electromagnetic radiation is dominant in 
the corresponding eigenmode. [This behavior is similar to that found at $\hat{k}=\hat{k}_{r}$ for Example 2 in Sec. III.C.] Figs. 5(a) and 9(a) are very similar at large values of $\hat{k}$ in conformity with previous analytic results pertaining to the large $\hat{k}$ behaviors Eq. (69) when $s_{1}=0$ and Eq. (100) when $w_{1}=0$.

Example 3: The analogue of Example 3 of Sec. III [whose stability properties are summarized in Figs. 6(a)-6(d)] is obtained by setting $w_{1}=-2 i / \pi$ and $w_{2}=0$ in Eq. (98). Growth-rate curves for $-1.25 \leq \hat{k} \leq 10$ are presented in Fig. 10(a). Details of the growthrate curves in the negative $\hat{k}$ interval $(-1.25 \leq \hat{k} \leq 0)$ are shown in Fig. 10(b). Corresponding plots of Re $\hat{\omega}$ vs. $\hat{k}$ (for $-1.25 \leq \hat{k} \leq 0$ ) are presented in Fig. 11(a). Plots of the Poynting ratio in Eq. (105) vs. $\hat{k}$ appear in Fig. 11(b). The component $E_{1 z}\left(\hat{k}+1 / \beta_{z}, \hat{\omega}\right)$ will not necessarily vanish for unstable eigenmodes of this system. Consequently, plots of $E_{1-}(\hat{k}, \hat{\omega}) / E_{1 z}\left(\hat{k}+1 / \beta_{z}, \hat{\omega}\right)$ vs. $\hat{k}$ for unstable modes are presented in Fig. 11(c). Letters show corresponding points in Fig. 10 and Fig. 11.

A detailed comparison of Figs. 10 and 11(a) gives the following description of the unstable eigenmodes. The components of an eigenmode pertaining to the short growth-rate curve segment $D R$ are two forward-traveling LHP electromagnetic waves [from $E_{1-}(\hat{k}, \hat{\omega})$ and $\left.E_{1+}\left(\hat{k}+2 / \beta_{z}, \hat{\omega}\right)\right]$ and a backward-traveling electrostatic wave [from $E_{1 z}\left(\hat{k}+1 / \beta_{z}, \hat{\omega}\right)$ ]. The components pertaining to the growth-rate curve segments $R E F, A B C G$, and $M N$ are a backward-traveling LHP electromagnetic wave [from $E_{1-}(\hat{k}, \hat{\omega})$ ], a forward-traveling LHP electromagnetic wave [from $E_{1+}\left(\hat{k}+2 / \beta_{z}, \hat{\omega}\right)$ ], and a forward traveling electrostatic wave [from $E_{1 z}\left(\hat{k}+1 / \beta_{z}, \hat{\omega}\right)$ ]. All other growth-rate curve segments have eigenmodes consisting of forward traveling LHP and RHP electromagnetic waves and a forward traveling electrostatic wave.

The most rapidly growing eigenmode of this system is that at point $C$ in Figs. 10(a) and 10(b). The components of this eigenmode are a backward-traveling LHP wave, a forward-traveling LHP wave, and a forward-traveling electrostatic wave. Reference to Figs. 11(b) and 11(c) shows that the backward-traveling component [which arises from $\left.E_{1-}(\hat{k}, \hat{\omega})\right]$ is the largest component both in amplitude and energy transfer. A similar situation was found for small $|\hat{k}|$ in Fig. 6(a) for the axial-dependent case except that the forward-traveling electromagnetic component was found to be RHP. 
Two branches of the growth-rate curves in Figs. 10(a) and 10(b) (MNO and $A B C G H I)$ show moderate growth rates at the resonance wavenumber $\hat{k}_{r}=4.94$. For both of these branches, Figs. 11(b) and 11(c) show that the eigenmode at $\hat{k}_{r}=4.94$ has a relatively large RHP electromagnetic component. Again we see that this behavior differs from that of the uncoupled system in Fig. 8(a), which shows no growth of RHP electromagnetic waves at the resonance wavenumber. The growth rates at $\hat{k}_{r}$ are approximately the same in Fig. 6(a) for the time-dependent equilibrium and Fig. 10(a) for the axial-dependent equilibrium; however, the electrostatic components of the corresponding eigenvectors are of greater relative amplitude in the axial-dependent case than in the time-dependent case. [Compare Fig. 11(c) with Fig. 6(d).]

Finally, at large values of $\hat{k}$, Figs. 10(a) and 6(a) approximate each other closely. This fact conforms with our previous results giving the large- $|\hat{k}|$ behaviors of Eq. (69) for $s_{2}=0$ and Eq. (100) for $w_{2}=0$. However, the corresponding eigenmodes $\left[\mathbf{E}\right.$ in Eq. (60) and $\mathbf{E}^{\prime}$ in Eq. (91)] are different even in the limit of large $\hat{k}$. By comparing Fig. 6(d) with Fig. 11(c), it is seen that (at large $\hat{k}$ ) the electrostatic component is relatively much stronger in the case of the axial-dependent equilibrium distribution.

Example 4: To obtain the analogue of Example 4 of Sec. III.C (whose stability properties are summarized in Fig. 7), choose $w_{1}=w_{2}=1$. These values are obtained by choosing $\Psi(\zeta)=\sum_{-\infty}^{\infty} \delta(\zeta-2 n \pi)$, so that in effect the phase of any particle is given by $\phi=m \Omega_{c} z / p_{z}$ ). Growth-rate curves (for $-1.254 \leq \hat{k} \leq 8$ ) and corresponding plots of Re $\hat{\omega}$ vs. $\hat{k}$ (for $-1.254 \leq \hat{k} \leq 0$ ) are presented in Figs. 12(a) and 12(b), respectively. For unstable branches of the dispersion relation in Eq. (100), plots of the Poynting flux ratio in Eq. (105) vs. $\hat{k}$ and $E_{1-}(\hat{k}, \hat{\omega}) / E_{1 z}\left(\hat{k}+1 / \beta_{z}, \hat{\omega}\right)$ vs. $\hat{k}$ are presented in Figs. $12(\mathrm{c})$ and 12(d), respectively. Letters show corresponding points in these plots. The letters correspond only loosely to those in Fig. 7.

Comparing Figs. 12(a) and 12(b), we see that the components of an eigenmode on growth-rate curve segment $D N$ are a backward-traveling LHP electromagnetic wave $\left[E_{1+}\left(\hat{k}+2 / \beta_{z}, \hat{\omega}\right)\right]$, a forward-traveling LHP electromagnetic wave $\left[E_{1-}(\hat{k}, \hat{\omega})\right]$, and a backward-traveling electrostatic wave $\left[E_{1 z}\left(\hat{k}+1 / \beta_{z}, \hat{\omega}\right)\right]$. Eigenmodes on growth-rate curve segments $A C B$ and $N E F$ consist of a backward-traveling LHP electromagnetic 
wave $\left[E_{1-}(\hat{k}, \hat{\omega})\right]$, a forward-traveling LHP electromagnetic wave $\left[E_{1+}\left(\hat{k}+2 / \beta_{z}, \hat{\omega}\right)\right]$, and a forward-traveling electrostatic wave $\left[E_{1 z}\left(\hat{k}+1 / \beta_{z}, \hat{\omega}\right)\right]$. Eigenmodes on all other growthrate curve segments consist of forward traveling RHP and LHP electromagnetic waves and a forward-traveling electrostatic wave.

Some properties of the growth-rate curves in Fig. 12(a) are similar to those in Fig. 7(a). Like Fig. 7(a), Fig. 12(a) shows no growth at large values of $\hat{k}$. Both sets of curves show very large growth rates at small values of $|\hat{k}|$, where backward waves occur [i.e., near point $B$ in Fig. 7(a) and point $C$ in Fig. 12(a)]. The eigenmode at point $C$ in Fig. 12(a) consists of a backward-traveling LHP electromagnetic wave [from $E_{1-}(\hat{k}, \hat{\omega})$ ], and forward-traveling LHP electromagnetic and electrostatic modes. Reference to Figs. 12(c) and 12(d) shows that the backward-traveling component exceeds the other two components in amplitude. In Sec. III.C, a similar situation was found to exist at point $B$ in Fig. 7(a), except that the forward-traveling electromagnetic component is RHP. Like Fig. 7(a), Fig. 12(a) shows a fairly large growth rate at the resonance wavenumber on the branch $A C B I J$. Moreover, Figs. $12(\mathrm{c})$ and $12(\mathrm{~d})$ show that the corresponding eigenmode has a relatively strong RHS electromagnetic component.

Finally, notice that no gap appears in the growth-rate curves in Fig. 12(a) to correspond to the gap between points $F$ and $H$ in Fig. $7(\mathrm{a})$.

\section{Remarks Concerning Numerical Examples.}

The analysis of the above numerical examples for the axial-dependent equilibrium leads to the same general conclusions as those given in Sec. III.C for the time-dependent equilibrium. A rich structure of different growth-rate curves and unstable eigenmodes can be induced by varying the form of $\Psi(\zeta)$, i.e., the values of $w_{1}$ and $w_{2}$. A suitable choice of $\Psi(\zeta)$ can significantly increase growth rates of RHP electromagnetic waves at the resonance wavenumber $\hat{k}=1 /\left(1-\beta_{z}\right)$ and can significantly increase or reduce growth rates at large values of $\hat{k}$. At small values of $\hat{k}$, where backward-traveling components are present, growth rates and the structures of eigenmodes depend strongly on the form of $\Psi(\zeta)$. This fact suggests that properties of absolute instabilities may depend strongly on $\Psi(\zeta)$. However, a study of this conjecture has not been carried out. 
For the same parameters $\left(\Omega_{c}, \gamma_{0}, \alpha_{0}\right)$, growth rate curves for corresponding $\left(s_{1}=w_{1}\right.$, $\left.s_{2}=w_{2}\right)$ time-dependent and axial-dependent systems usually show some resemblance. Nevertheless, the eigenmode structures are very different in the two cases. In the timedependent case, unstable eigenmodes for coupled systems consist of two or three components of the same wavenumbers and different frequencies, whereas in the axial-dependent case the components have the same frequencies and different wavenumbers. Moreover, for small values of $\hat{k}$, the handedness and directions of motion of components may differ between the two cases. 


\section{Conclusions}

We have studied stability properties of a relativistic electron beam propagating along an applied magnetic field $B_{0} \hat{\mathbf{e}}_{\mathbf{z}}$, using the Maxwell-Vlasov equations under the constraint that spatially dependent quantities are functions of $z$ only. Of particular interest are those cases in which the equilibrium distribution is not uniformly random in the electron gyro-phase angle $\phi$. Two equilibrium distributions have been considered. These are the time-dependent distribution $f_{0}\left(p_{\perp}, p_{z}, \xi\right)$, where $\xi=\phi-\Omega_{c} t / \gamma$, and the spatial-dependent distribution $f_{0}\left(p_{\perp}, p_{z}, \zeta\right)$, where $\zeta=\phi-m \Omega_{c} z / p_{z}$. Since neither of these distributions can be converted into the other by a Lorentz transformation, the distributions represent two physically different systems. It is found that in general the Fourier components of the perturbed electric and magnetic fields are related the integral equations (26)-(28) for the case of the time-dependent equilibrium distribution, and by the integral equations (41)-(43) for the case of the spatial-dependent equilibrium distribution. In our numerical analysis, however, we consider special cases in which the integral equations reduce to algebraic equations even though the equilibrium distribution is not uniformly random in phase.

If there is no spread in electron energies (or equivalently $p$ ) in the time-dependent equilibrium distribution, then the integral equations (26)-(28) reduce to just three algebraic equations [Eq. (58)] relating the Fourier components $E_{1-}(k, \omega), E_{1+}\left(k, \omega-2 \omega_{c}\right)$, and $E_{1 z}\left(k, \omega-\omega_{c}\right)$ of the perturbed fields. Consequently, an eigenmode of the system consists of a RHP electromagnetic wave, a LHP electromagnetic wave, and an electrostatic wave. These components have the same wavenumber, and the same spatial and temporal growth or decay rates, but have different frequencies. [The electrostatic component is decoupled if the Fourier coefficient $\hat{g}_{1}\left(p_{0}, \alpha\right)$ in Eq. (57) vanishes, and all three components decouple if $\hat{g}_{2}\left(p_{0}, \alpha\right)$ also vanishes.]

If there is no spread in the axial component of momentum $\left(p_{z}\right)$ in the spatial-dependent equilibrium distribution, then the integral equations (41)-(43) reduce to just three algebraic equations [Eq. (89)] relating the perturbed field Fourier components $E_{1-}(k, \omega)$, $E_{1+}\left(k+2 m \Omega_{c} / p_{z 0}, \omega\right), E_{1 z}\left(k+m \Omega_{c} / p_{z 0}, \omega\right)$. Therefore, the components of an eigenmode are a RHP electromagnetic wave, a LHP electromagnetic wave, and an electrostatic wave. 
These components have the same frequency, and the same spatial and temporal growth or decay rates, but have different wavenumbers. [In analogy with the time-dependent case, the electrostatic component is decoupled if the Fourier coefficient $\hat{h}_{1}\left(p_{\perp}, p_{z 0}\right)$ in Eq. (88) vanishes, and all of the components decouple if $\hat{h}_{2}\left(p_{\perp}, p_{z 0}\right)$ also vanishes.]

Numerical computations of stability properties have been carried out for both the timeand spatial-dependent equilibrium distributions for the case where no spread is present in both $p$ and the pitch angle $\alpha$ (or equivalently in both $p_{\perp}$ and $p_{z}$ ). In this case the frequencies and wavenumbers can be normalized to the relativistic cyclotron frequency $\omega_{c}$ by defining $\hat{\omega}=\omega / \omega_{c}$ and $\hat{k}=c k / \omega_{c}$. The computations are restricted to real values of $\hat{k}$, so that $\operatorname{Im} \hat{\omega}>0$ indicates temporal growth. It is found that (for fixed applied magnetic field, energy, and pitch angle) a rich variety of growth-rate curves and eigenmodes can be obtained by changing the dependence of the equilibrium distribution on the phase angle. Appropriate choices of the phase-angle dependence can significantly increase growth rates near the resonance wave number $\hat{k}_{r}=1 /\left(1-\beta_{z}\right)$. Growth rates at large values of $\hat{k}$ can on the one hand be enhanced and on the other hand be suppressed altogether. Moreover, finite intervals (in $\hat{k}$ ) of no growth can be produced. Finally, growth rate curves and the form of eigenvectors at small values of $|\hat{k}|$, where backward traveling components are present, are particularly sensitive to the $\phi$-dependence of the equilibrium distribution.

As an important area in our current research, we are analyzing the integral equations to determine the structures of eigenmodes in the general case.

\section{Acknowledgement}

This research was supported in part by the Air Force Office of Scientific Research, Grant Numbers F49620-94-1-0374 and F49620-97-1-0325. 


\section{References}

1. K. R. Chu and J. L. Hirschfield, Phys. Fluids 21, 461 (1978).

2. R. C. Davidson and P. H. Yoon, Phys. Rev. A 39, 2534 (1989).

3. P. H. Yoon and R. C. Davidson, Phys. Rev. A 35, 2619 (1987).

4. P. H. Yoon and R. C. Davidson, Phys. Rev. A 35, 2718 (1987).

5. P. H. Yoon and R. C. Davidson, J. Plasma Physics 43, 269 (1990).

6. H. S. Uhm and R. C. Davidson, Phys. Fluids 29, 2713 (1986).

7. A. Fruchtman and L. Friedland, J. Appl. Phys. 53, 4011 (1982).

8. A. Fruchtman and L. Friedland, IEEE J. Quantum Electron. 19327 (1983).

9. T. H. Kho and A. T. Lin and L. Chen, Phys. Fluids 31, 3120 (1988).

10. C. Chen, J. A. Davies, G. Zhang, and J. Wurtele, Phys. Rev. Lett. 69, 73 (1992).

11. P. Yoon and T. Chang, J. Plasma Physics 42, 193 (1989).

12. V. L. Bratman, N. S. Ginsberg, G. S. Nusinovich, M. I. Petelin and P. S. Strelkov, Int. J. Electron. 61, 541 (1981).

13. T. H. Kho, A. T. Lin, Phys. Fluids B 2, 822 (1990).

14. K. R. Chen, J. M. Dawson, A. T. Lin, and T. Katsouleas, Phys. Fluids B 3, 1270 (1991).

15. B. G. Danly, J. A. Davies, K. D. Pendergast, R. J. Tempkin, and J. S. Wurtele, Proc. SPIE, Microwave and Particle Beam Sources and and Directed Energy Concepts, 1061, 243 (1989).

16. C. Chen and J. S. Wurtele, Phys. Rev. Lett. 653389 (1990).

17. C. Chen and J. S. Wurtele, Phys. Fluids B 3, 2133 (1991). 
18. C. Chen, B. G. Danly, G. Shevets and J. S. Wurtele, IEEE Trans. Plasma Sci. 20, 149 (1992).

19. H. P. Freund and C. Chen, Int. J. Electronics 72, 1005 (1992).

20. C. S. Wu, Space Science Reviews 41, 215 (1985).

21. H. P. Freund, C. S. Wu and J. D. Gaffey, Jr., Phys. Fluids 27, 1396 (1984).

22. H. P. Freund, J. Q. Dong, C. S. Wu and L. C. Lee, Phys. Fluids 30, 3106 (1987).

23. P. H. Yoon and D. Krauss-Varban, Phys. Fluids B 2, 1918 (1990).

24. P. H. Yoon and C. S. Wu, Phys. Rev. A 44, 6819 (1991).

25. F. Ziebelland P. H. Yoon, Phys. Plasmas 2, 1285 (1995).

26. A. Bers, Handbook of Plasma Physics, vol 1. Basic Plasma Physics I (ed.'A. A. Galeev and R. N. Sudan) North Holland, Chap. 3.2 (1983).

27. Y. Y. Lau, K. R. Chu, L. R. Barnett, and V. I. Granatstein, Int. J. Infrared and Millimeter Waves 2, 373-393 (1981).

28. J. A. Davies, Phys. Fluids B 1, 663 (1989). 


\section{Appendix. Relations between Equilibrium Distributions}

In this paper, we consider equilibrium distributions whose form in the laboratory reference frame is either $f_{0}(\mathbf{p}, t)=f_{0}\left(p_{\perp}, p_{z}, \xi\right)$ or $f_{0}(z, \mathbf{p})=f_{0}\left(p_{\perp}, p_{z}, \zeta\right)$. Below it is shown that, under a Lorentz transformation to a new reference frame moving in the $z$ direction relative to the laboratory frame with velocity $\beta_{u}=u / c$, either of these forms is transformed into combinations of the two original forms.

It is well known that a distribution $f_{0}(z, \mathbf{p}, t)$ is invariant under a Lorentz transformation. Consequently, under the Lorentz transformations described above the the distribution becomes

$$
f_{0}^{\prime}\left(z^{\prime}, \mathbf{p}^{\prime}, t^{\prime}\right)=f_{0}(z, \mathbf{p}, t)
$$

where

$$
\begin{aligned}
z & =\gamma_{u}\left(z^{\prime}+\beta_{u} c t^{\prime}\right), \\
c t & =\gamma_{u}\left(c t^{\prime}+\beta_{u} z^{\prime}\right), \\
p_{z} & =\gamma_{u}\left(p_{z}^{\prime}+\beta_{u} \gamma^{\prime} m c\right), \\
\gamma m c & =\gamma_{u}\left(\gamma^{\prime} m c+\beta_{u} p_{z}^{\prime}\right) .
\end{aligned}
$$

Moreover, $p_{\perp}=p_{\perp}^{\prime}, \phi=\phi^{\prime}$, and $\gamma_{u}=\left(1-\beta_{u}^{2}\right)^{-\frac{1}{2}}$.

Expressing $\xi=\Omega_{c} t / \gamma$ in terms of transformed (primed) quantities, we find that

$$
\begin{aligned}
\xi & =\left(1+\beta_{u} \beta_{z}^{\prime}\right)^{-1} \xi^{\prime}+\beta_{z}^{\prime} \beta_{u}\left(1+\beta_{u} \beta_{z}^{\prime}\right)^{-1} \zeta^{\prime} \\
& =\gamma_{u}^{2}\left(1-\beta_{u} \beta_{z}\right) \xi^{\prime}+\gamma_{u}^{2} \beta_{u}\left(\beta_{z}-\beta_{u}\right) \zeta^{\prime}
\end{aligned}
$$

where $\beta_{z}=v_{z} / c$, and

$$
\begin{aligned}
& \xi^{\prime}=\phi-\frac{\Omega_{c}}{\gamma^{\prime}} t^{\prime}, \\
& \zeta^{\prime}=\phi-\frac{\Omega_{c}}{\gamma^{\prime}} \frac{z^{\prime}}{v_{z}^{\prime}}=\phi-\frac{m \Omega_{c}}{p_{z}^{\prime}} z^{\prime} .
\end{aligned}
$$

Consequently, if the laboratory frame distribution is of the form $f_{0}(\mathbf{p}, t)=$ $f_{0}\left(p_{\perp}, p_{z}, \xi\right)$, then the moving frame distribution will be of the form $f_{0}^{\prime}\left(z^{\prime}, \mathbf{p}^{\prime}, t^{\prime}\right)=$ 
$f_{0}\left(p_{\perp}, p_{z}^{\prime}, c_{1} \xi^{\prime}+c_{2} \zeta^{\prime}\right)$, where $c_{1}=\left(1+\beta_{u} \beta_{z}^{\prime}\right)^{-1}$ and $c_{2}=\beta_{z}^{\prime} \beta_{u}\left(1+\beta_{u} \beta_{z}^{\prime}\right)^{-1}$. Notice that $c_{1}+c_{2}=1$ and that $c_{1}>0$, so that a Lorentz transformation cannot change the form of a distribution from $f_{0}\left(p_{\perp}, p_{z}, \xi\right)$ into $f_{0}^{\prime}\left(p_{\perp}, p_{z}^{\prime}, \zeta^{\prime}\right)$ for any value of $\mathbf{p}^{\prime}$. Finally, notice that Eq. (55) must be applied with care to the singular case of $\beta_{u}=\beta_{z}$ (i.e., $\beta_{z}^{\prime}=0$ ), because $\zeta^{\prime} \longrightarrow \infty$ as $\beta_{z}^{\prime} \longrightarrow 0$. In this singular case, $\xi=\xi^{\prime}-\beta_{z} \Omega_{c} z^{\prime} / \gamma^{\prime} c$ where $\gamma^{\prime}=\left(p_{\perp}^{2} / m^{2} c^{2}+1\right)^{1 / 2}$.

Expressing $\zeta=\phi-m \Omega_{c} z / \gamma p_{z}=\phi-\Omega_{c} z / \gamma v_{z}$ in terms of primed quantities, we obtain

$$
\begin{aligned}
\zeta & =\frac{\beta_{u}}{\left(\beta_{u}+\beta_{z}^{\prime}\right)} \xi^{\prime}+\frac{\beta_{z}^{\prime}}{\left(\beta_{u}+\beta_{z}^{\prime}\right)} \zeta^{\prime} \\
& =\frac{\gamma_{u}^{2} \beta_{u}}{\beta_{z}}\left(1-\beta_{z} \beta_{u}\right) \xi^{\prime}+\frac{\gamma_{u}^{2}}{\beta_{z}}\left(\beta_{z}-\beta_{u}\right) \zeta^{\prime} .
\end{aligned}
$$

Under a Lorentz transformation, a distribution of the form $f_{0}(z, \mathbf{p})=f_{0}\left(p_{\perp}, p_{z}, \zeta\right)$ attains the form $f_{0}^{\prime}\left(z^{\prime}, \mathbf{p}^{\prime}, t^{\prime}\right)=f_{0}^{\prime}\left(p_{\perp}, p_{z}^{\prime}, c_{1} \xi^{\prime}+c_{2} \zeta^{\prime}\right)$, where now $c_{1}=\beta_{u} /\left(\beta_{u}+\beta_{z}^{\prime}\right)$ and $c_{2}=$ $\beta_{z}^{\prime} /\left(\beta_{u}+\beta_{z}^{\prime}\right)$. Again, notice that $c_{1}+c_{2}=1$. In the singular case of $\beta_{u} \longrightarrow \beta_{z}$ (i. e., $\beta_{z}^{\prime} \longrightarrow 0$ ), Eq. (A6) reduces to $\zeta=\xi^{\prime}-\frac{\Omega_{c}}{\gamma^{\prime}} \frac{z^{\prime}}{v_{z}}$. Consequently, a Lorentz transformation cannot change the form of a distribution from $f_{0}\left(p_{\perp}, p_{z}, \zeta\right)$ into $f_{0}^{\prime}\left(p_{\perp}, p_{z}^{\prime}, \xi^{\prime}\right)$ for any value of $\mathbf{p}^{\prime}$. 


\section{Figure Captions}

1. The phase angle $\phi$ and the pitch angle $\alpha$ of the single particle momentum $\mathbf{p}$.

2. Schematic diagrams of (a) the time-dependent phase distribution at a single instant of time for the case where $\xi=0$ for all particles, and (b) of the axial dependent distribution for all time for the case where $\zeta=0$ for all particles.

3. Distributions in phase $\Phi(\xi)$ used in numerical examples. Plot (a) is a uniformly random (gyrotropic) distribution. Also shown are plots of nongyrotropic distributions for which (b) $s_{1}=0$ and $s_{2}=\sin a / a$, (c) $s_{1}=-4 i K$ and $s_{2}=0$, and (d) $s_{1}=$ $(2 / a) \exp (-i a / 2) \sin (a / 2)$ and $s_{2}=(1 / a) \exp (-i a) \sin a$.

4. Plots of complex $\hat{\omega}$ vs. $\hat{k}$ (real) for the system parameters $\omega_{c}^{2} / \omega_{p}^{2}=0.05, \gamma_{0}=2$, and $\alpha_{0}=0.4$. The time-dependent equilibrium distribution in phase is characterized by $s_{1}=s_{2}=0$. Plots are (a) $\operatorname{Im} \hat{\omega}$ vs. $\hat{k}$ for $0 \leq \hat{k} \leq 10$ and (b) Re $\hat{\omega}$ vs. $\hat{k}$ for $0 \leq \hat{k} \leq 1.4$.

5. Dispersion relations and properties of corresponding eigenvectors for system parameters $\omega_{c}^{2} / \omega_{p}^{2}=0.05, \gamma_{0}=2$, and $\alpha_{0}=0.4$. The time-dependent equilibrium distribution in phase is characterized by $s_{1}=0$ and $s_{2}=2 \sqrt{2} / \pi$. Plots are (a) $\operatorname{Im} \hat{\omega}$ vs. $\hat{k}$ for $0 \leq \hat{k} \leq 15$ and (b) Re $\hat{\omega}$ vs. $\hat{k}$ for $0 \leq \hat{k} \leq 1.5$. Also shown for unstable eigenmodes is (c) the Poynting flux ratio in Eq. (85) vs. $\hat{k}$.

6. Dispersion relations and properties of corresponding eigenvectors for system parameters $\omega_{c}^{2} / \omega_{p}^{2}=0.05, \gamma_{0}=2$, and $\alpha_{0}=0.4$. The time-dependent equilibrium distribution in phase is characterized by $s_{1}=-2 i / \pi$ and $s_{2}=0$. Plots are (a) $\operatorname{Im} \hat{\omega}$ vs. $\hat{k}$ for $0 \leq \hat{k} \leq 10$ and (b) Re $\hat{\omega}$ vs. $\hat{k}$ for $0 \leq \hat{k} \leq 2$. Also shown for unstable eigenmodes are (c) the Poynting flux ratio in Eq. (85) vs. $\hat{k}$ and (d) $2^{-1 / 2} \mid E_{1-}(\hat{k}, \hat{\omega}) / E_{1 z}(\hat{k}, \hat{\omega}-1)$ vs. $\hat{k}$.

7. Dispersion relations and properties of corresponding eigenvectors for system parameters $\omega_{c}^{2} / \omega_{p}^{2}=0.05, \gamma_{0}=2$, and $\alpha_{0}=0.4$. The time-dependent equilibrium distribution in phase is characterized by $s_{1}=s_{2}=1$. Plots are (a) $\operatorname{Im} \hat{\omega}$ vs. $\hat{k}$ for $0 \leq \hat{k} \leq 8$ and (b) $\operatorname{Re} \hat{\omega}$ vs. $\hat{k}$ for $0 \leq \hat{k} \leq 1.5$. Also shown for unstable eigenmodes are (c) the Poynting flux ratio in Eq. (85) vs. $\hat{k}$ and (d) $2^{-1 / 2} \mid E_{1-}(\hat{k}, \hat{\omega}) / E_{1 z}(\hat{k}, \hat{\omega}-1)$ vs. $\hat{k}$.

8. Plots of complex $\hat{\omega}$ vs. $\hat{k}$ (real) for the system parameters $\omega_{c}^{2} / \omega_{p}^{2}=0.05, \gamma_{0}=2$, and 
$\alpha_{0}=0.4$. The spatial-dependent equilibrium distribution in phase is characterized by $w_{1}=w_{2}=0$. Plots are (a) $\operatorname{Im} \hat{\omega}$ vs. $\hat{k}$ for $-1.254 \leq \hat{k} \leq 10$ and (b) Re $\hat{\omega}$ vs. $\hat{k}$ for $-1.254 \leq \hat{k} \leq 0$.

9. Dispersion relations and properties of corresponding eigenvectors for system parameters $\omega_{c}^{2} / \omega_{p}^{2}=0.05, \gamma_{0}=2$, and $\alpha_{0}=0.4$. The spatial-dependent equilibrium distribution in phase is characterized by $w_{1}=0$ and $w_{2}=2 \sqrt{2} / \pi$. Plots are (a) Im $\hat{\omega}$ vs. $\hat{k}$ for $-1.254 \leq \hat{k} \leq 14$ and (b) Re $\hat{\omega}$ vs. $\hat{k}$ for $-1.254 \leq \hat{k} \leq 0$. Also shown for unstable eigenmodes is (c) the Poynting flux ratio in Eq. (105) vs. $\hat{k}$.

10. Growth-rate curves $\left(\operatorname{Im} \hat{\omega}\right.$ vs. $\hat{k}$, real) for system parameters $\omega_{c}^{2} / \omega_{p}^{2}=0.05, \gamma_{0}=2$, and $\alpha_{0}=0.4$. The spatial-dependent equilibrium distribution in phase is characterized by $w_{1}=-2 i / \pi$ and $w_{2}=0$. Plots are (a) $\operatorname{Im} \hat{\omega}$ vs. $\hat{k}$ for $-1.254 \leq \hat{k} \leq 10$ and (b) a detail of the previous plot for $-1.254 \leq \hat{k} \leq 0$.

11. Additional properties of the system whose growth-rate curves are plotted in Fig. 10. Plot (a) of Re $\hat{\omega}$ vs. $\hat{k}$ for $-1.254 \leq \hat{k} \leq 0$. Shown for unstable eigenmodes are (b) the Poynting flux ratio in Eq. (105) vs. $\hat{k}$ and (c) $2^{-1 / 2} \mid E_{1-}(\hat{k}, \hat{\omega}) / E_{1 z}\left(\hat{k}+1 / \beta_{z}, \hat{\omega}\right)$ vs. $\hat{k}$.

12. Dispersion relations and properties of corresponding eigenvectors for system parameters $\omega_{c}^{2} / \omega_{p}^{2}=0.05, \gamma_{0}=2$, and $\alpha_{0}=0.4$. The time-dependent equilibrium distribution in phase is characterized by $w_{1}=w_{2}=1$. Plots are (a) $\operatorname{Im} \hat{\omega}$ vs. $\hat{k}$ for $-1.254 \leq \hat{k} \leq 8$ and (b) Re $\hat{\omega}$ vs. $\hat{k}$ for $-1.254 \leq \hat{k} \leq 0$. Also shown for unstable eigenmodes are (c) the Poynting flux ratio in Eq. (105) vs. $\hat{k}$ and (d) $2^{-1 / 2} \mid E_{1-}(\hat{k}, \hat{\omega}) / E_{1 z}\left(\hat{k}+1 / \beta_{z}, \hat{\omega}\right)$ vs. $\hat{k}$. 


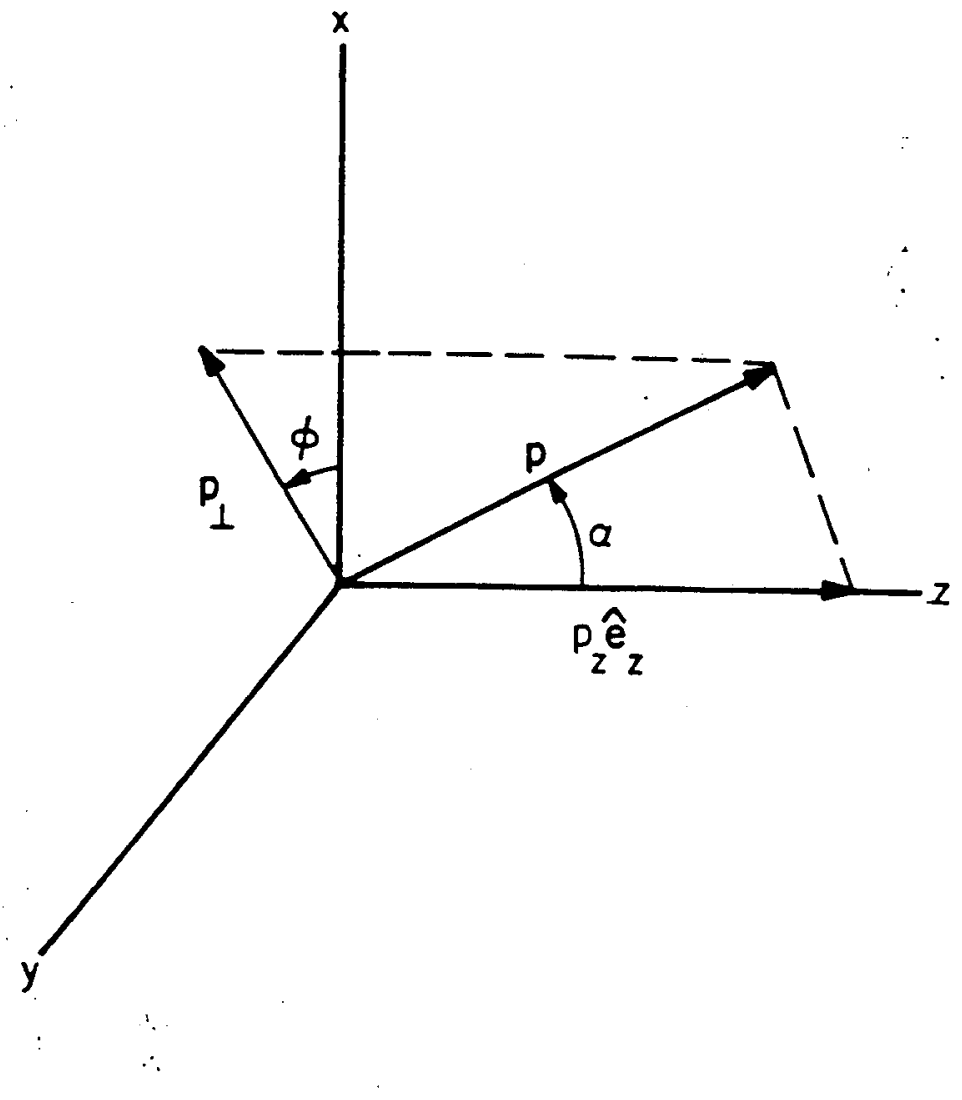

Figure 1

Davies, Phys. Plasmas 
(a)
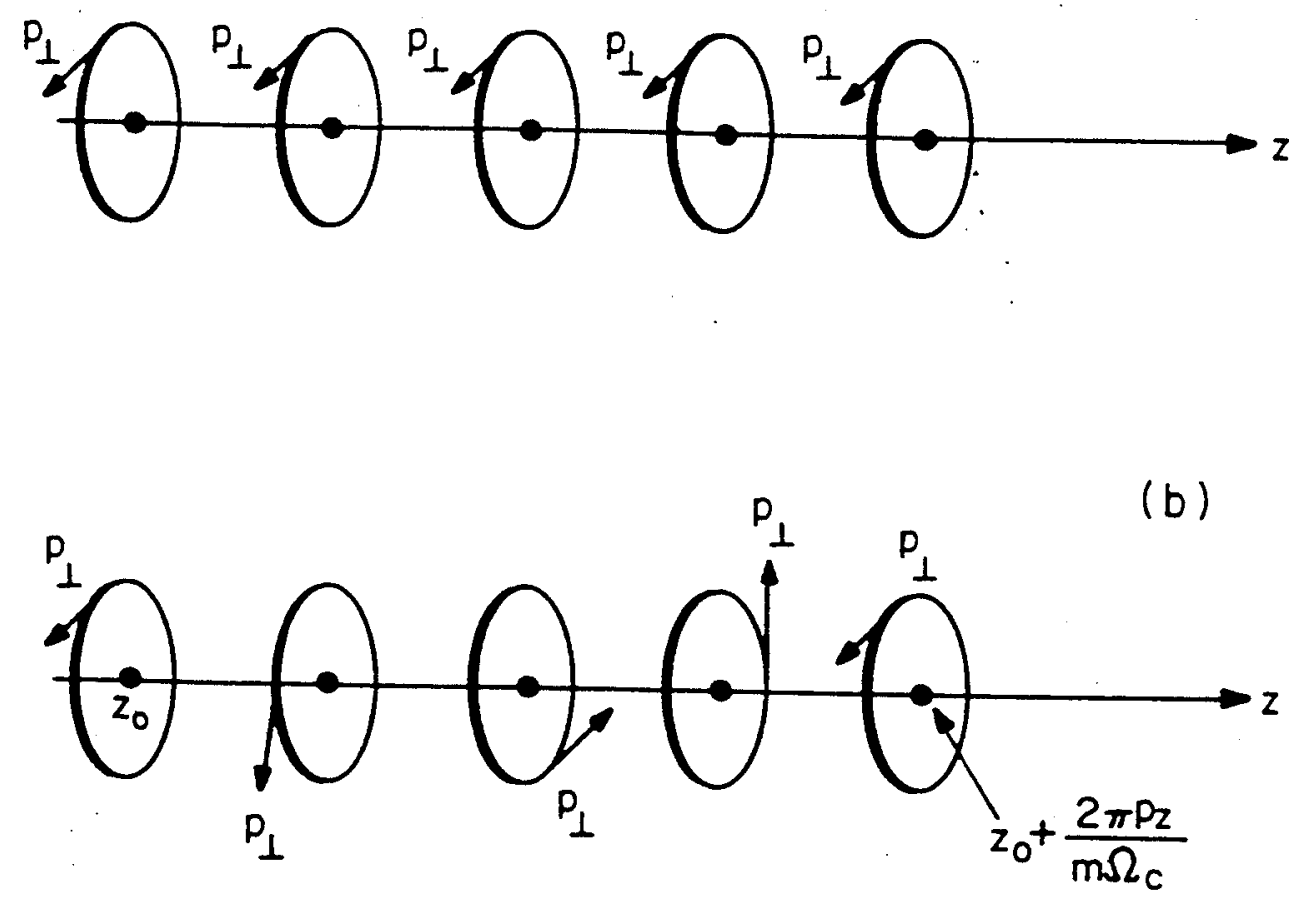

Figure 2

Davies, Phys. Plasmas 

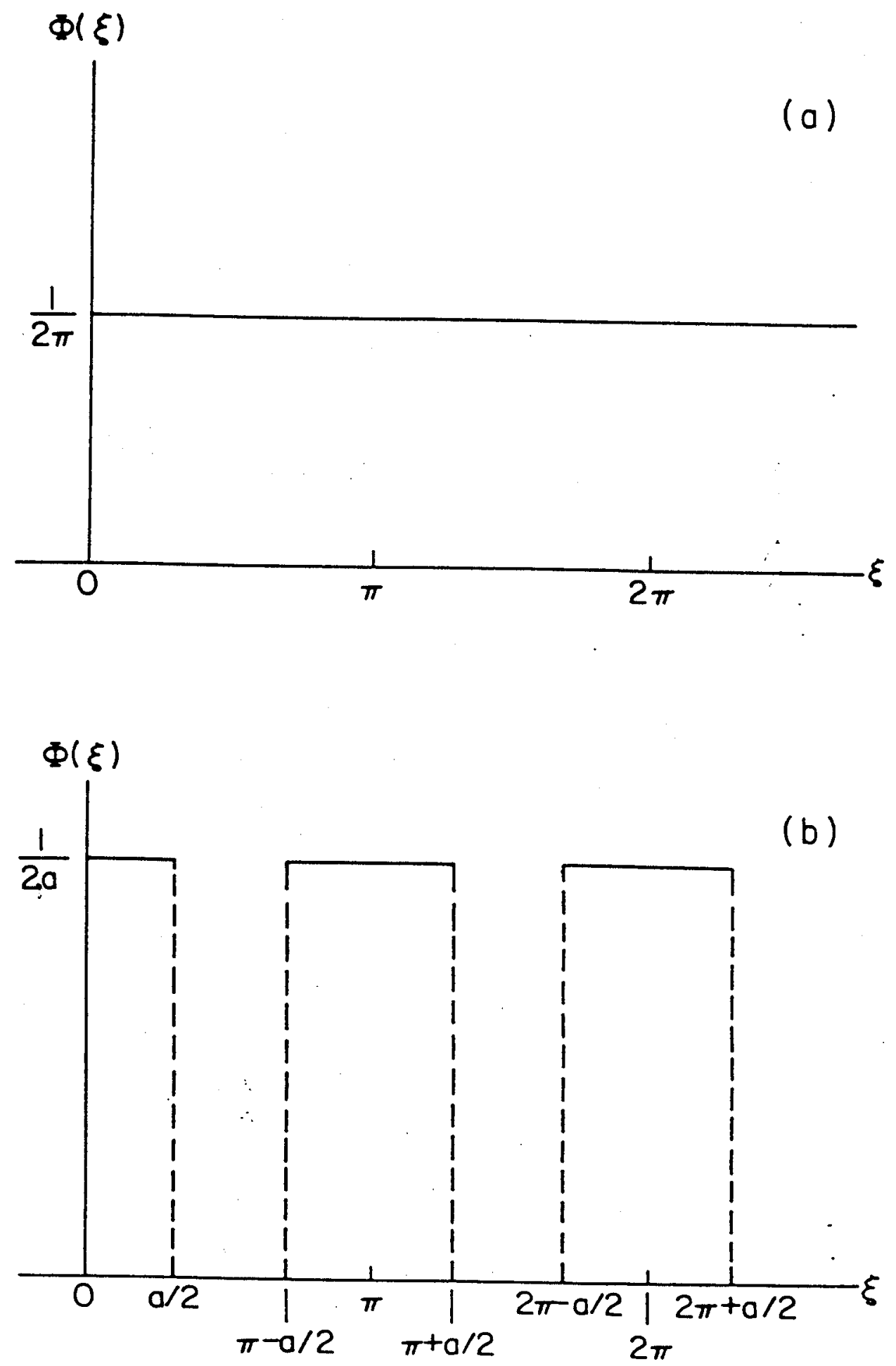

Figures 3(a) and 3(b)

Davies, Phys. Plasmas 

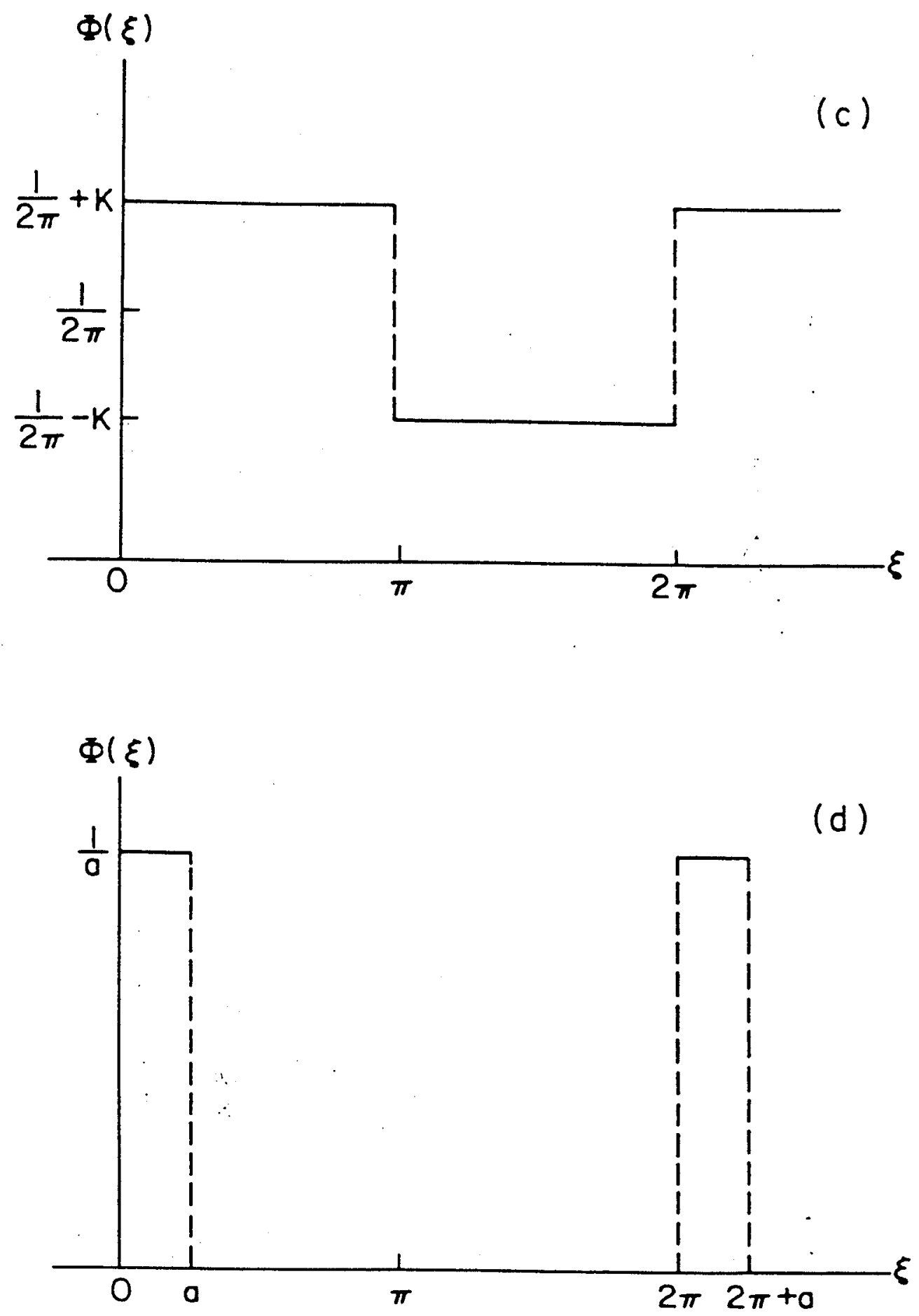

Figures 3(c) and 3(d)

Davies, Phys. Plasinas 


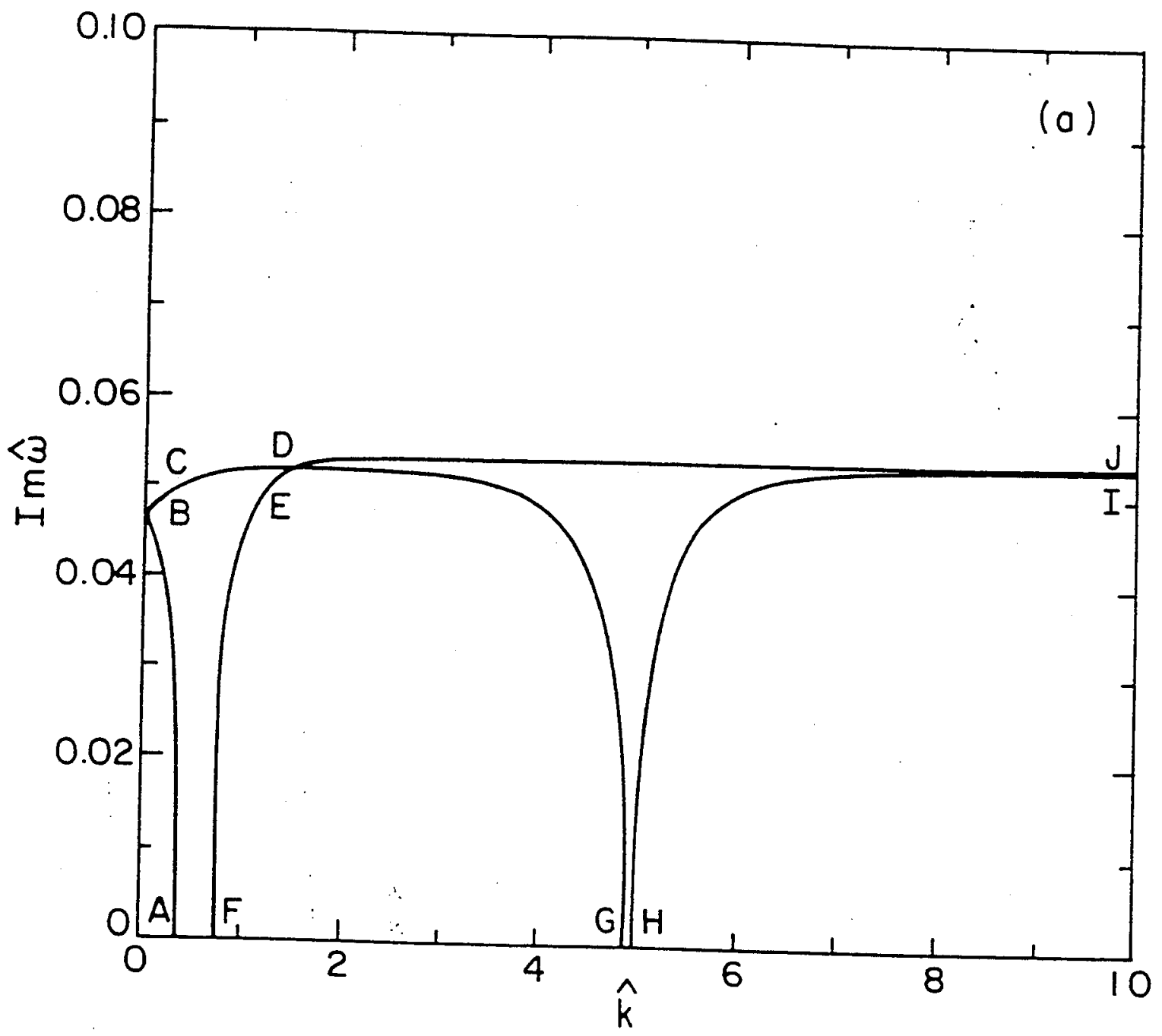

Figure 4(a)

Davies, Phys. Plasmas 


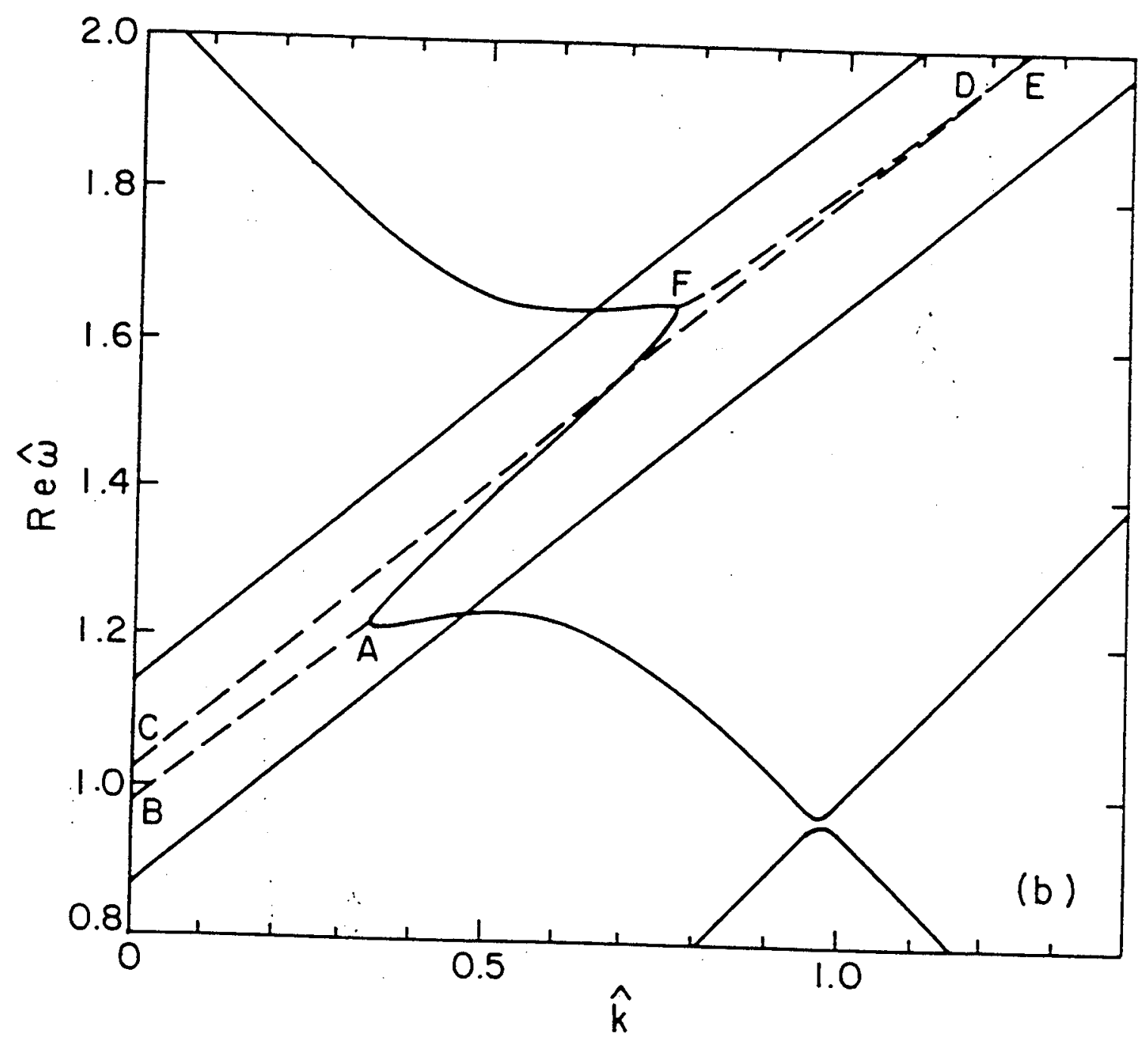

Figure 4(b)

Davies, Phys. Plasmas 


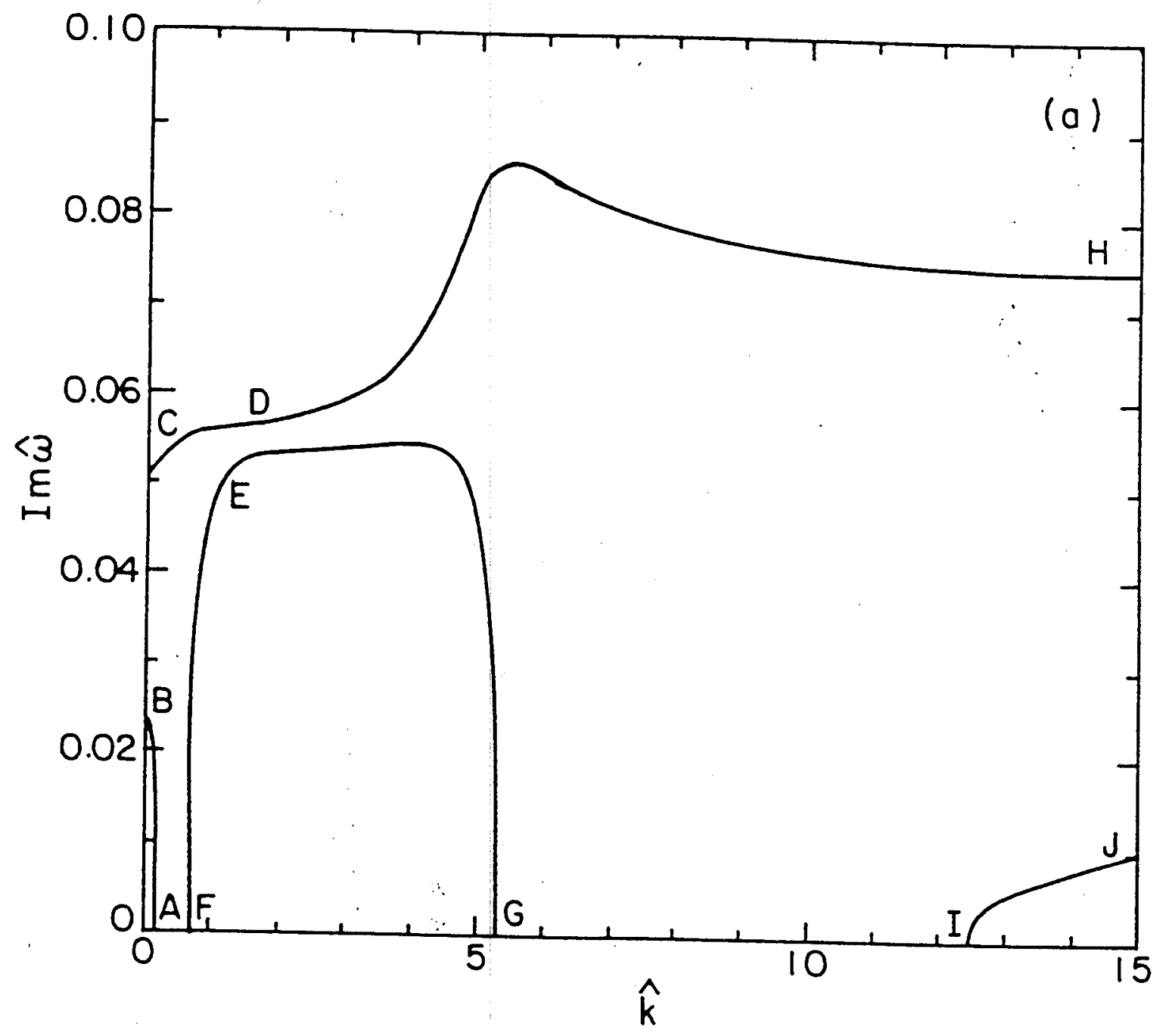

Figure 5(a)

Davies, Phys. Plasmas 


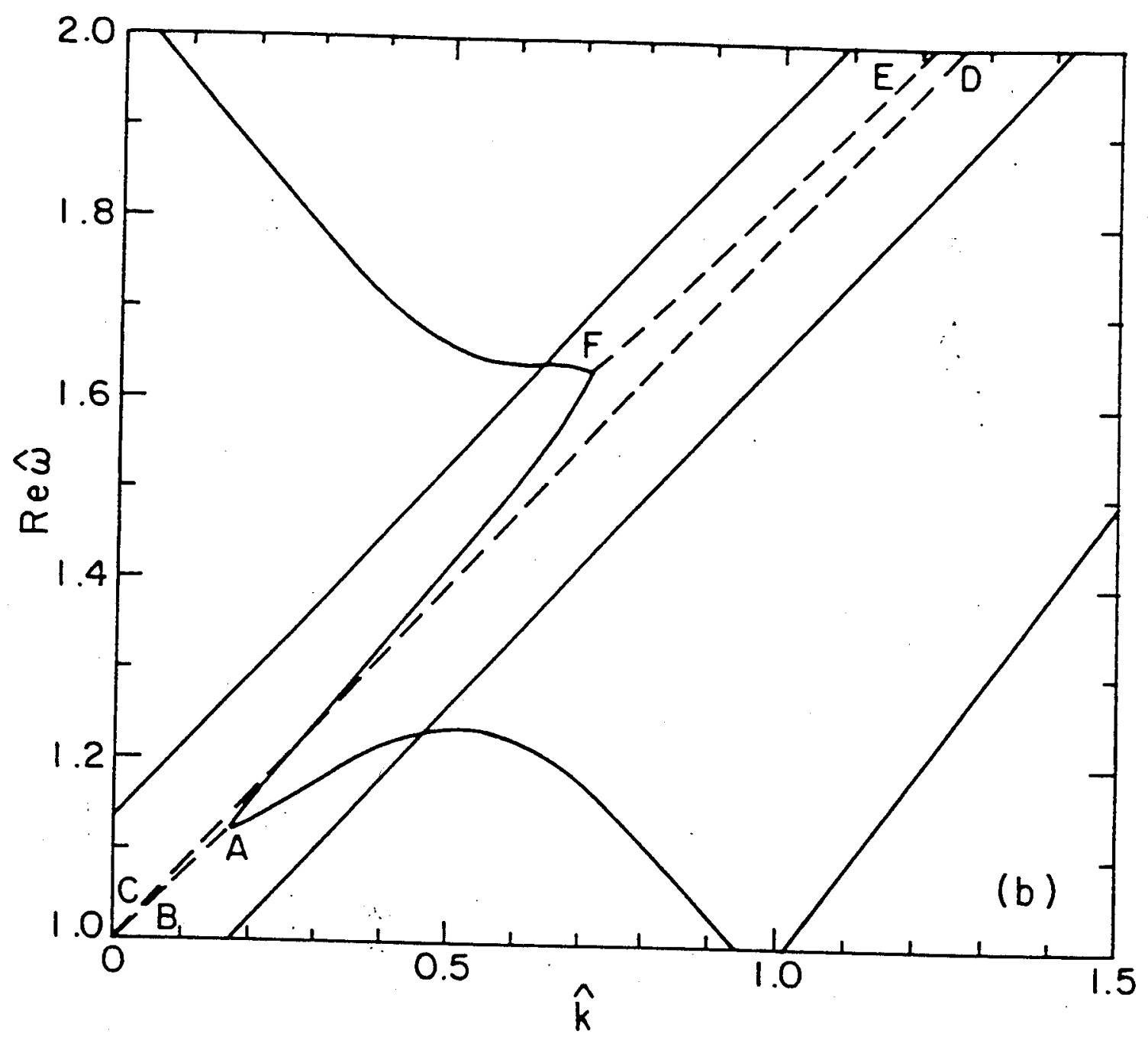

Figure 5(b)

Davies, Phys. Plasmas 


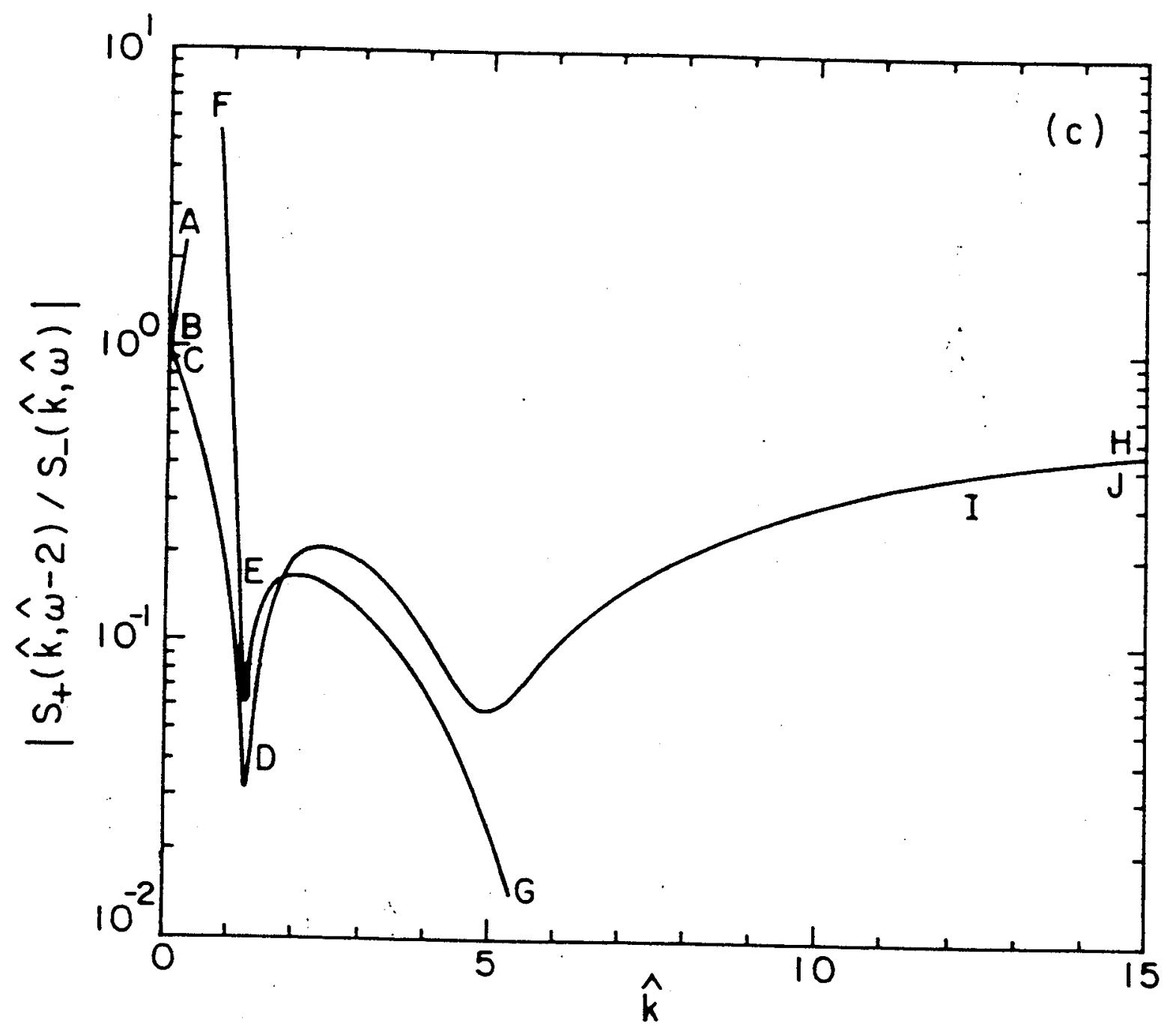

Figure 5(c)

Davies, Phys. Plasmas 


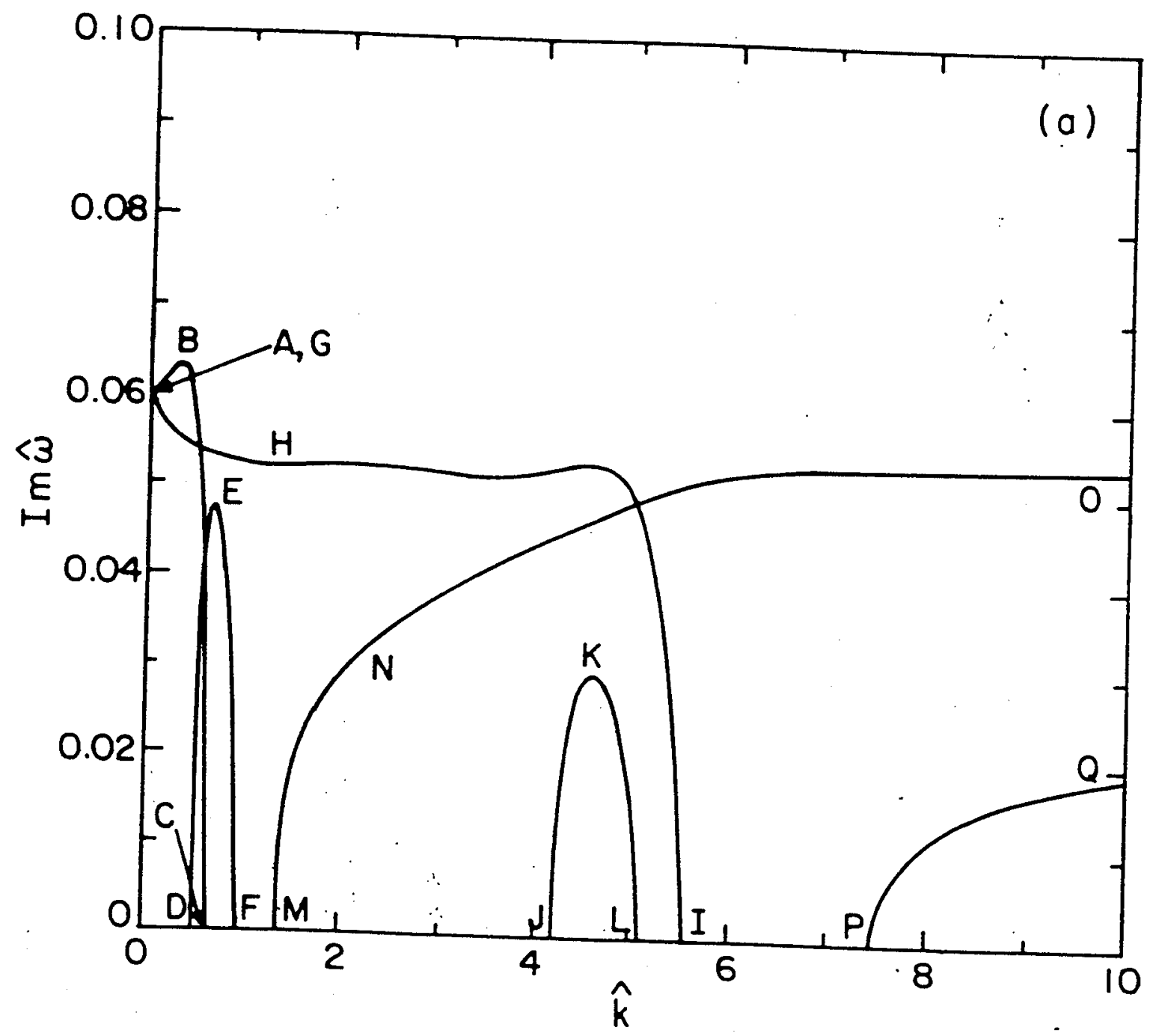

Figure 6(a)

Davies, Phys. Plasinas 


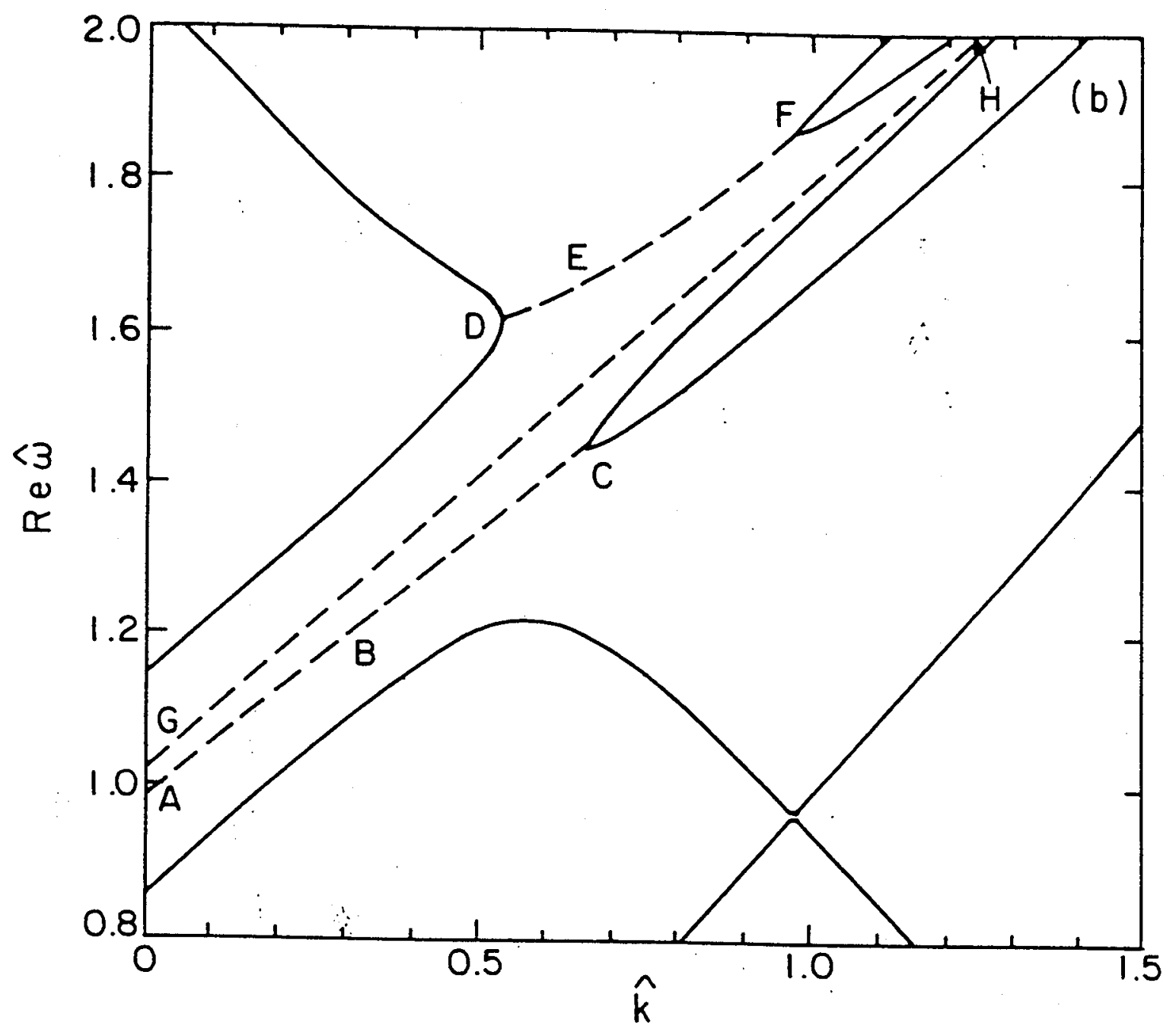

Figure 6(b)

Davies, Phys. Plasmas 


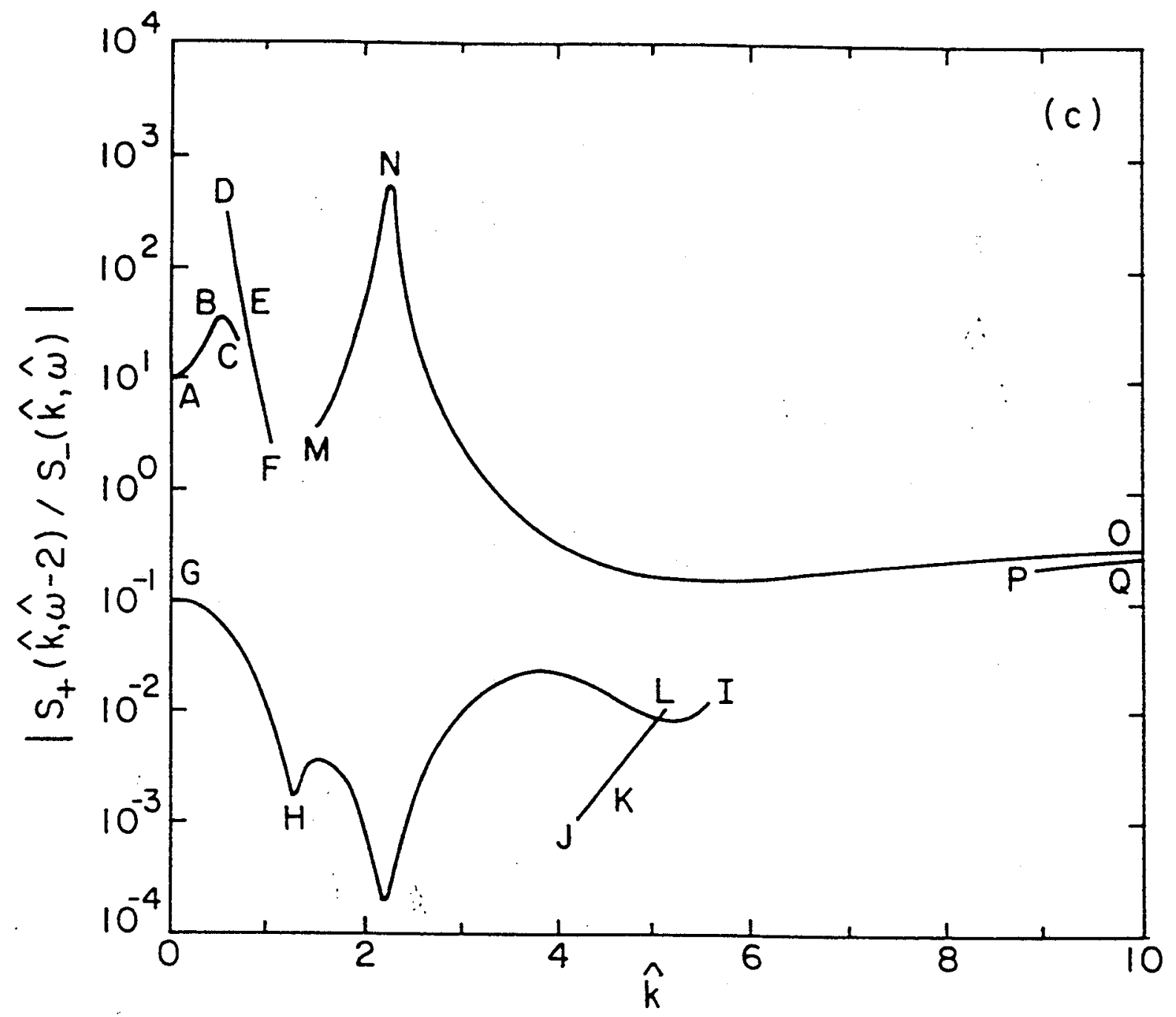

Figure 6(c)

Davies, Phys. Plasmas 


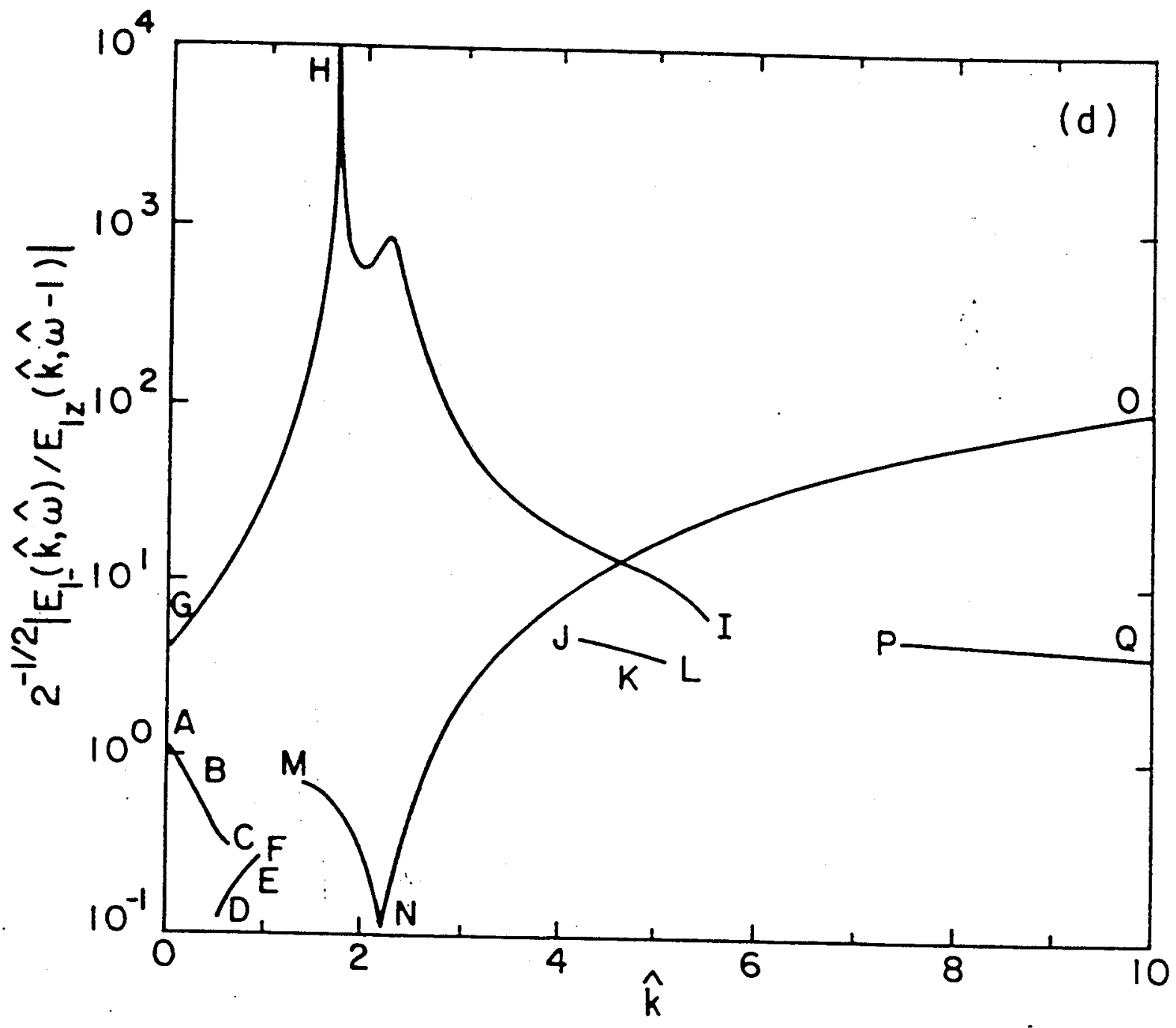

Figure 6(d)

Davies, Phys. Plasmas 


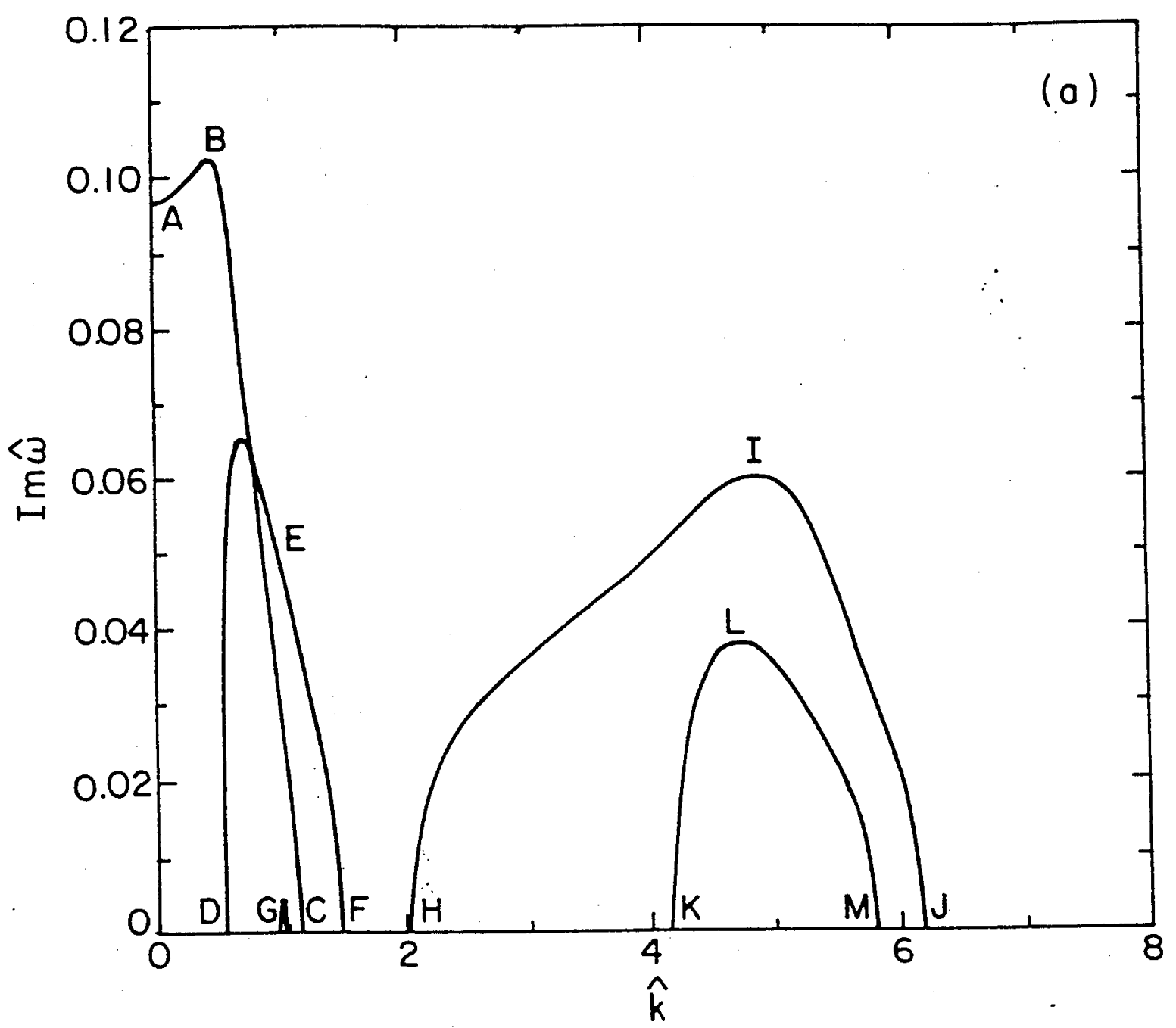

Figure 7(a)

Davies, Phys. Plasmas 


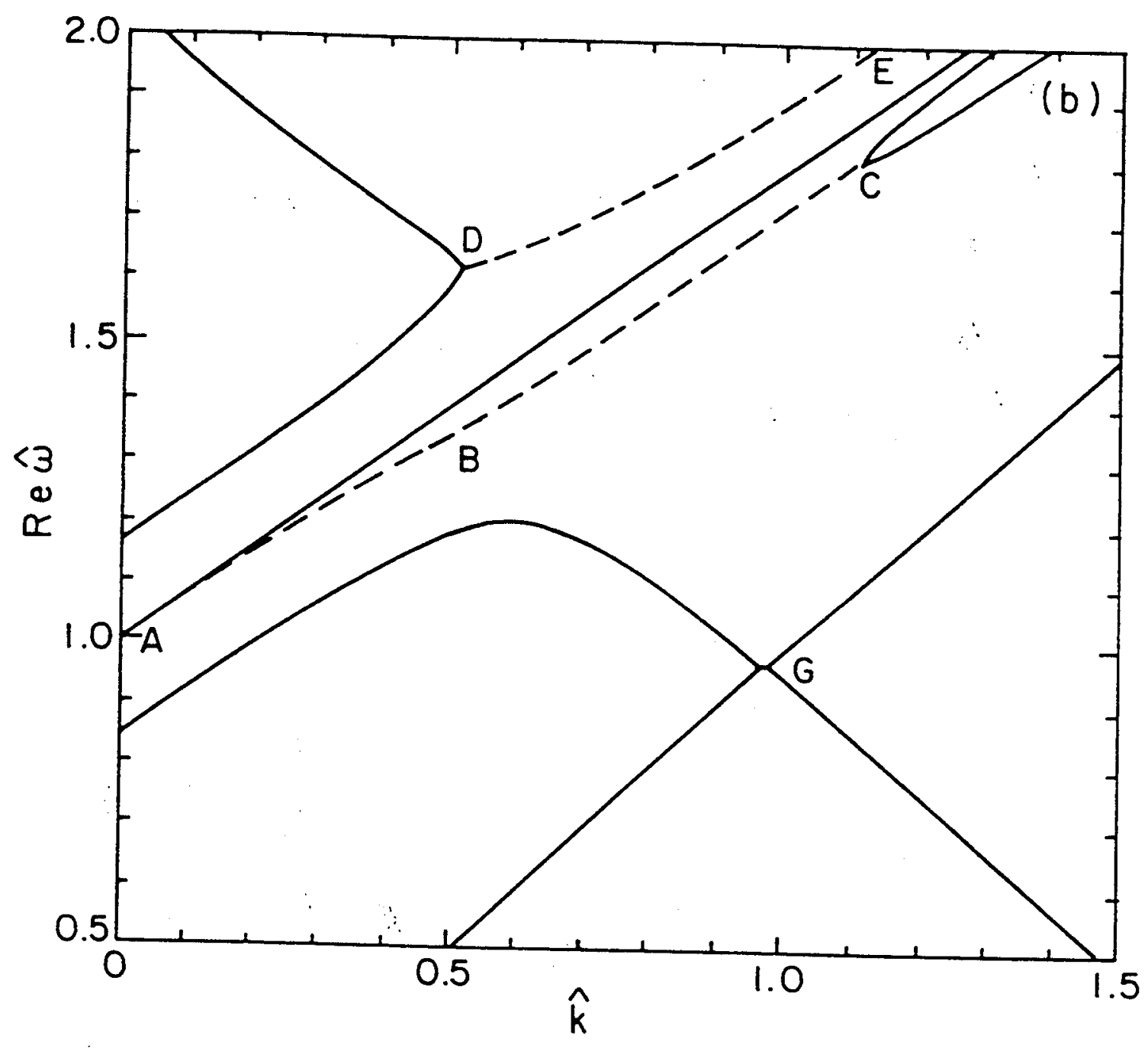

Figure 7(b)

Davies, Phys. Plasinas 


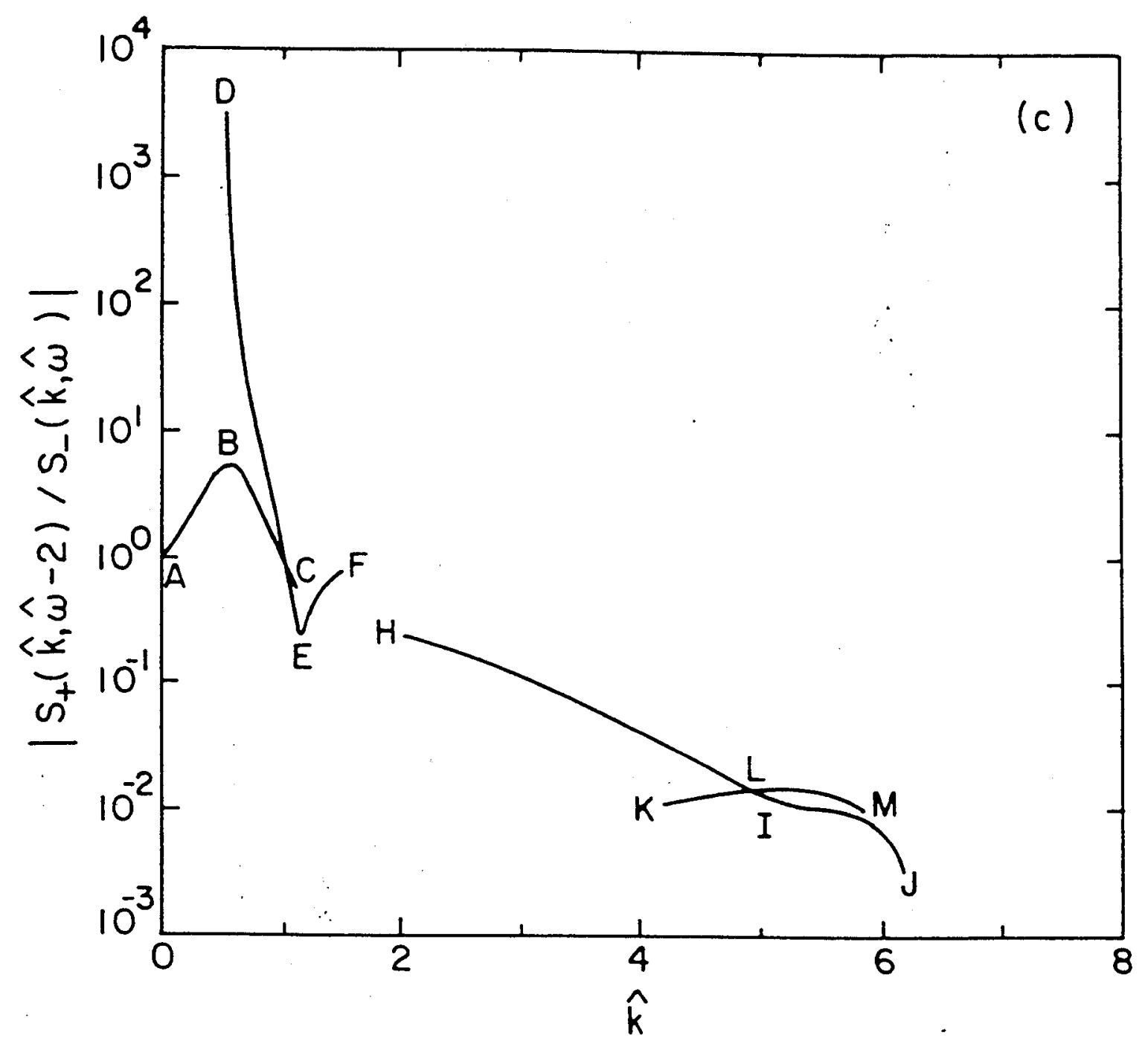

Figure 7(c)

Davies, Phys. Plasmas 


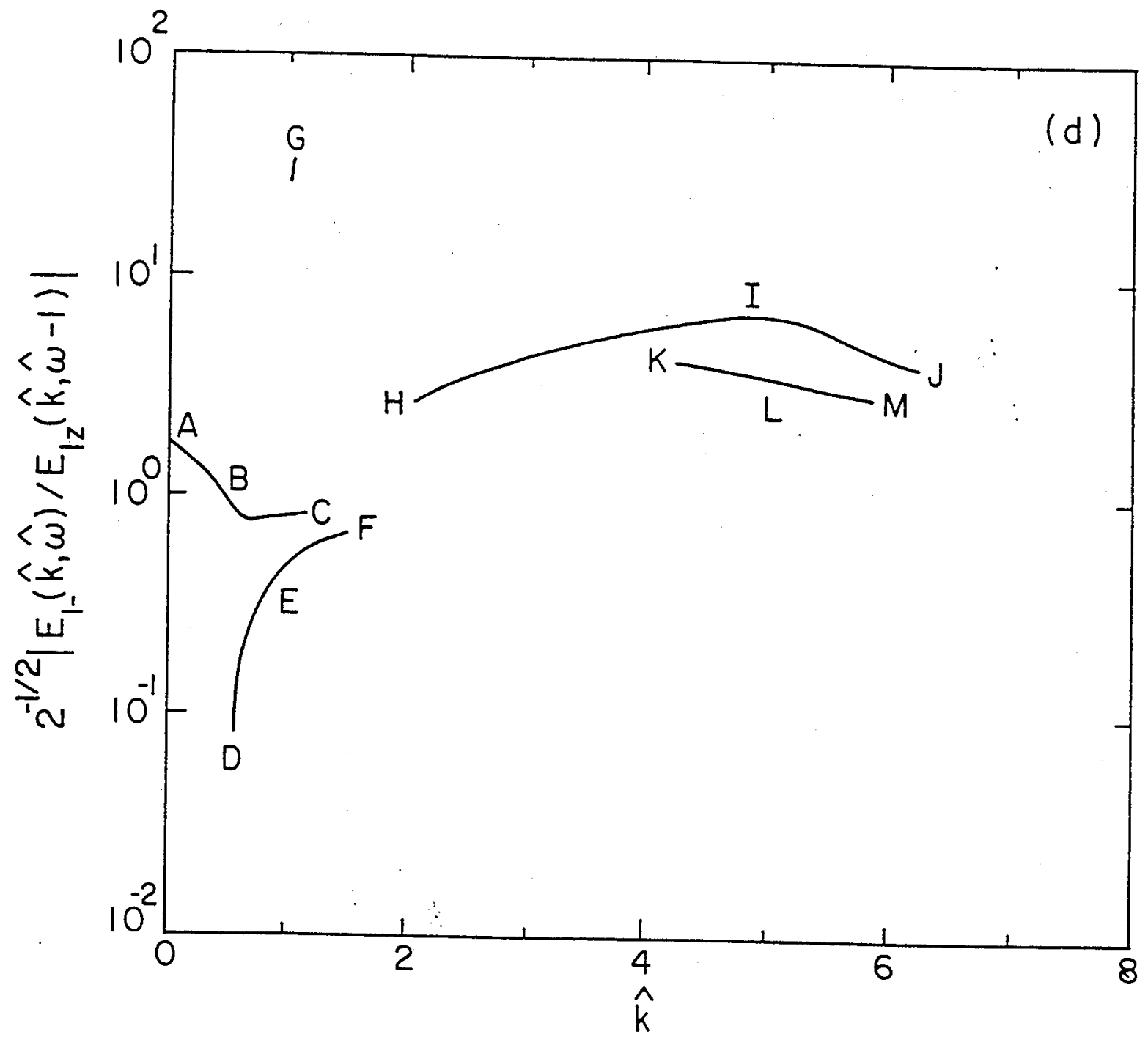

Figure 7(d)

Davies, Phys. Plasmas 


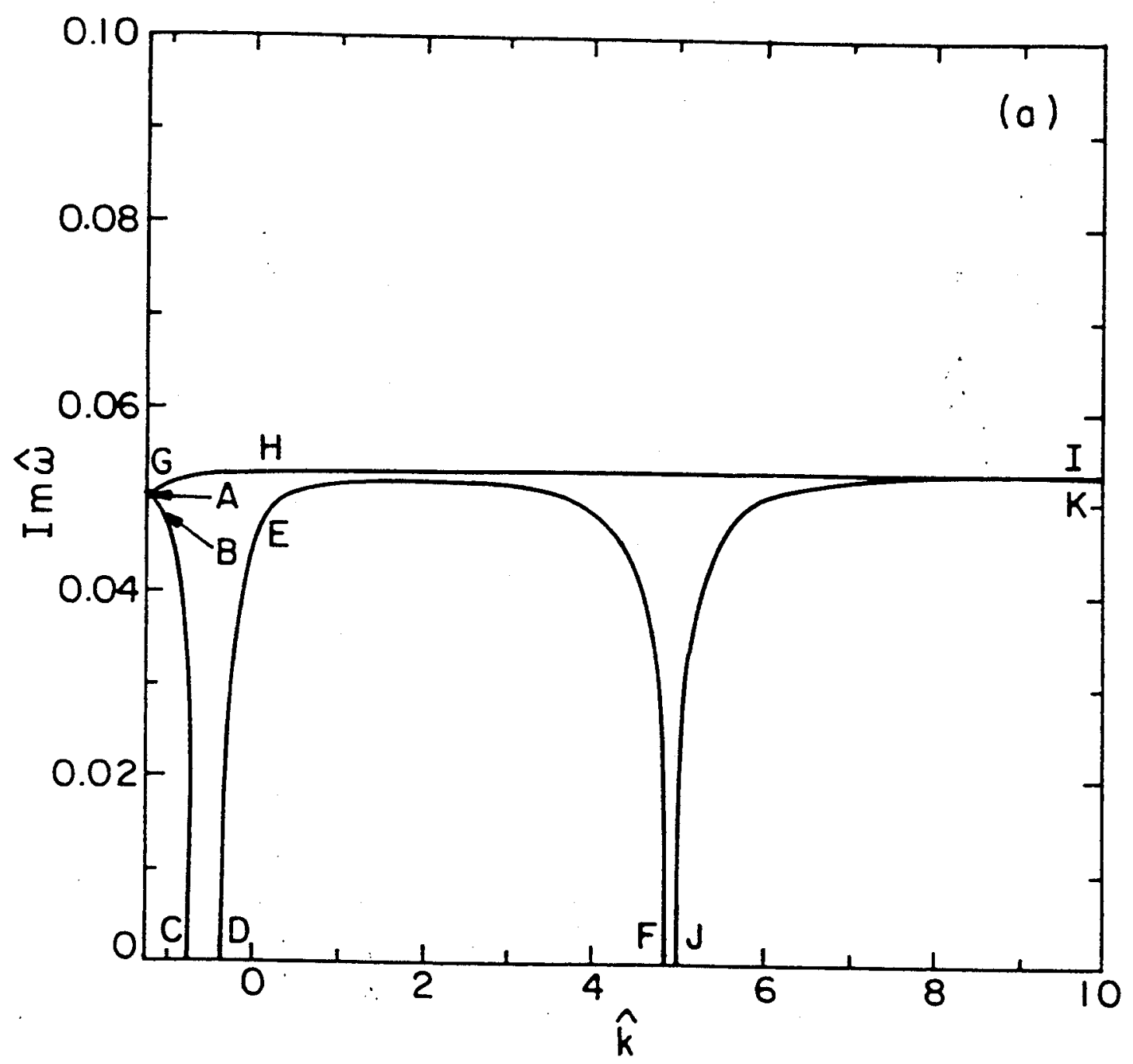

Figure 8(a)

Davies, Phys. Plasmas 


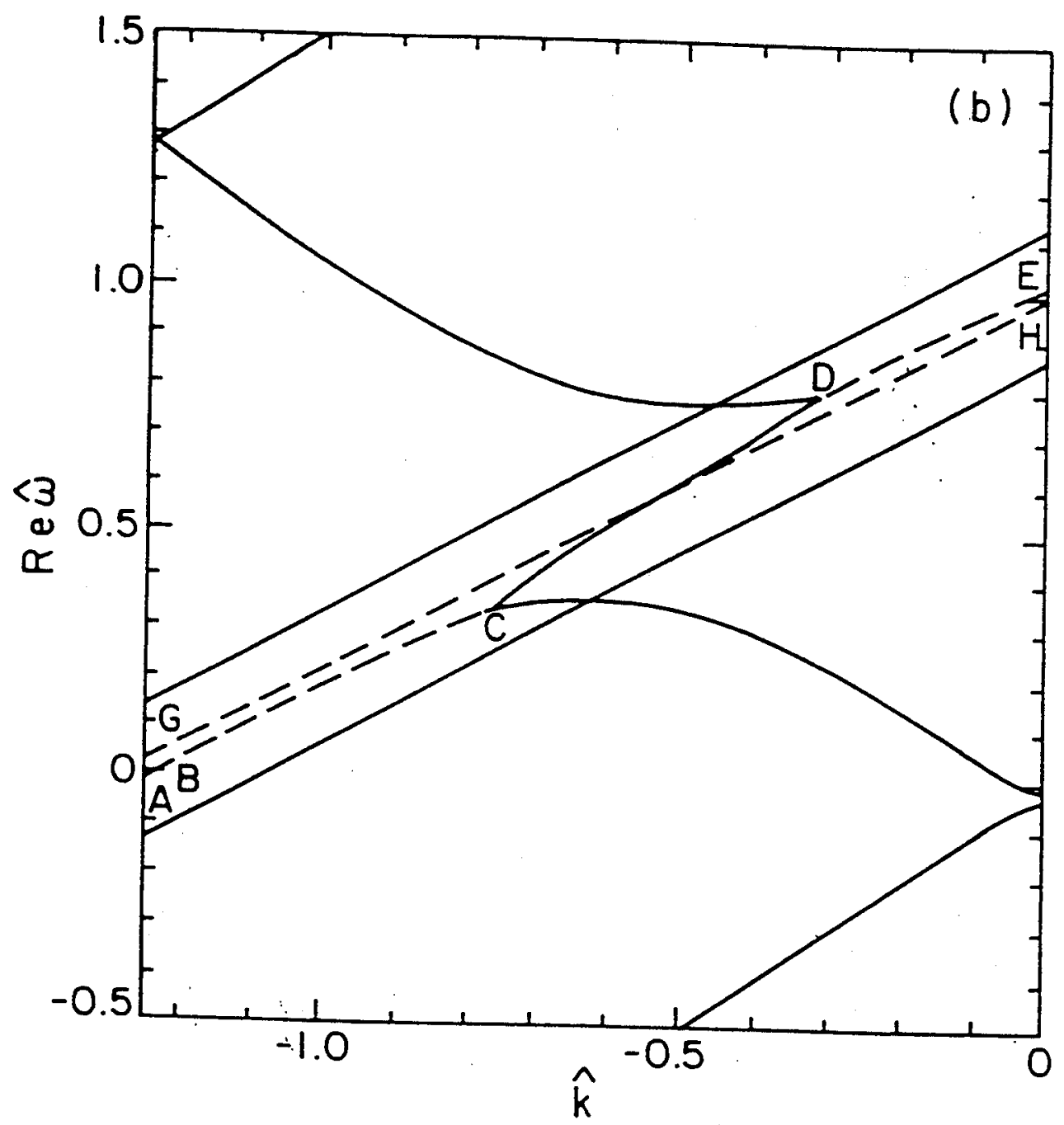

Figure 8(b)

Davies, Phys. Plasmas 


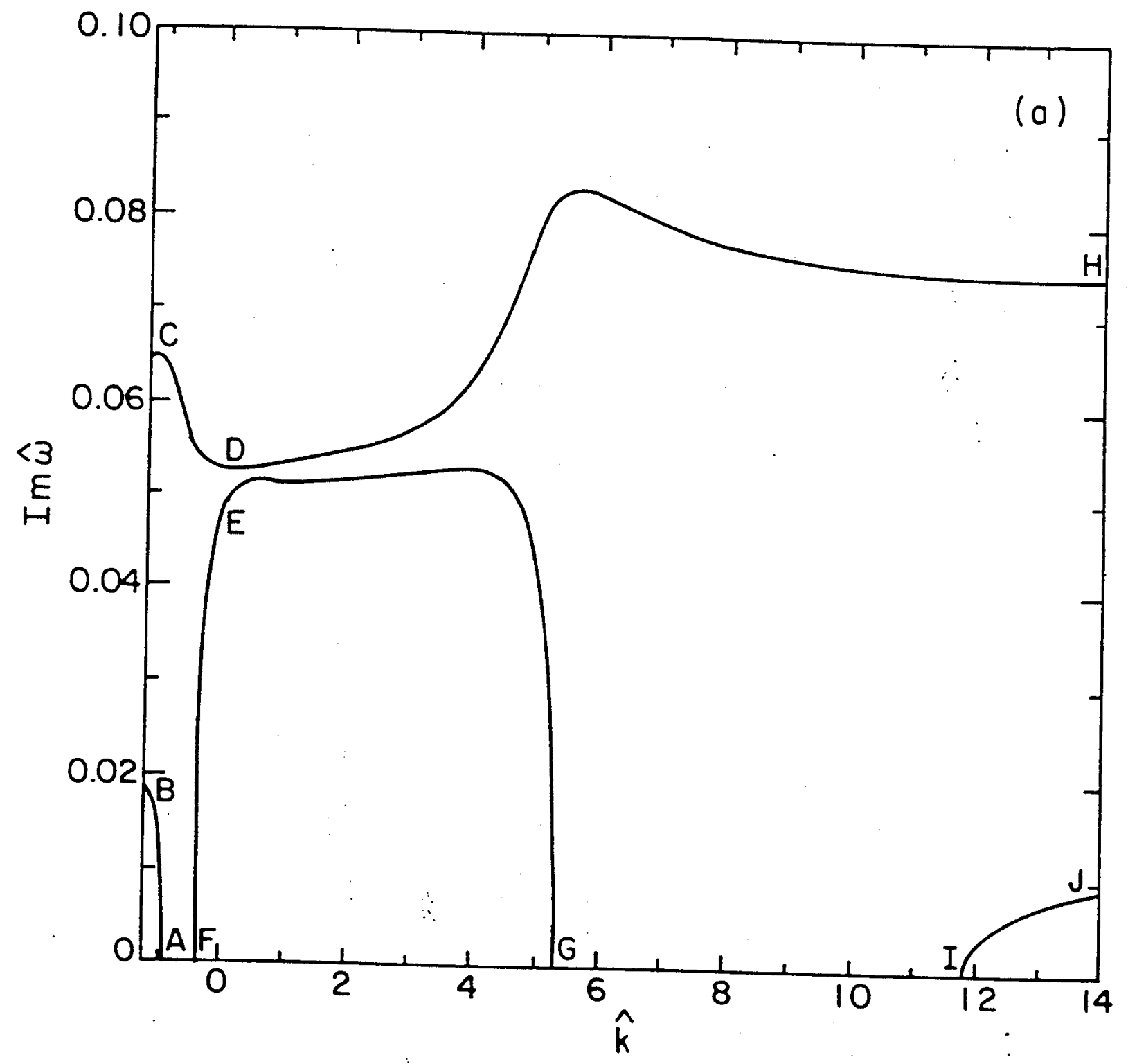

Figure 9(a)

Davies, Phys. Plasmas 


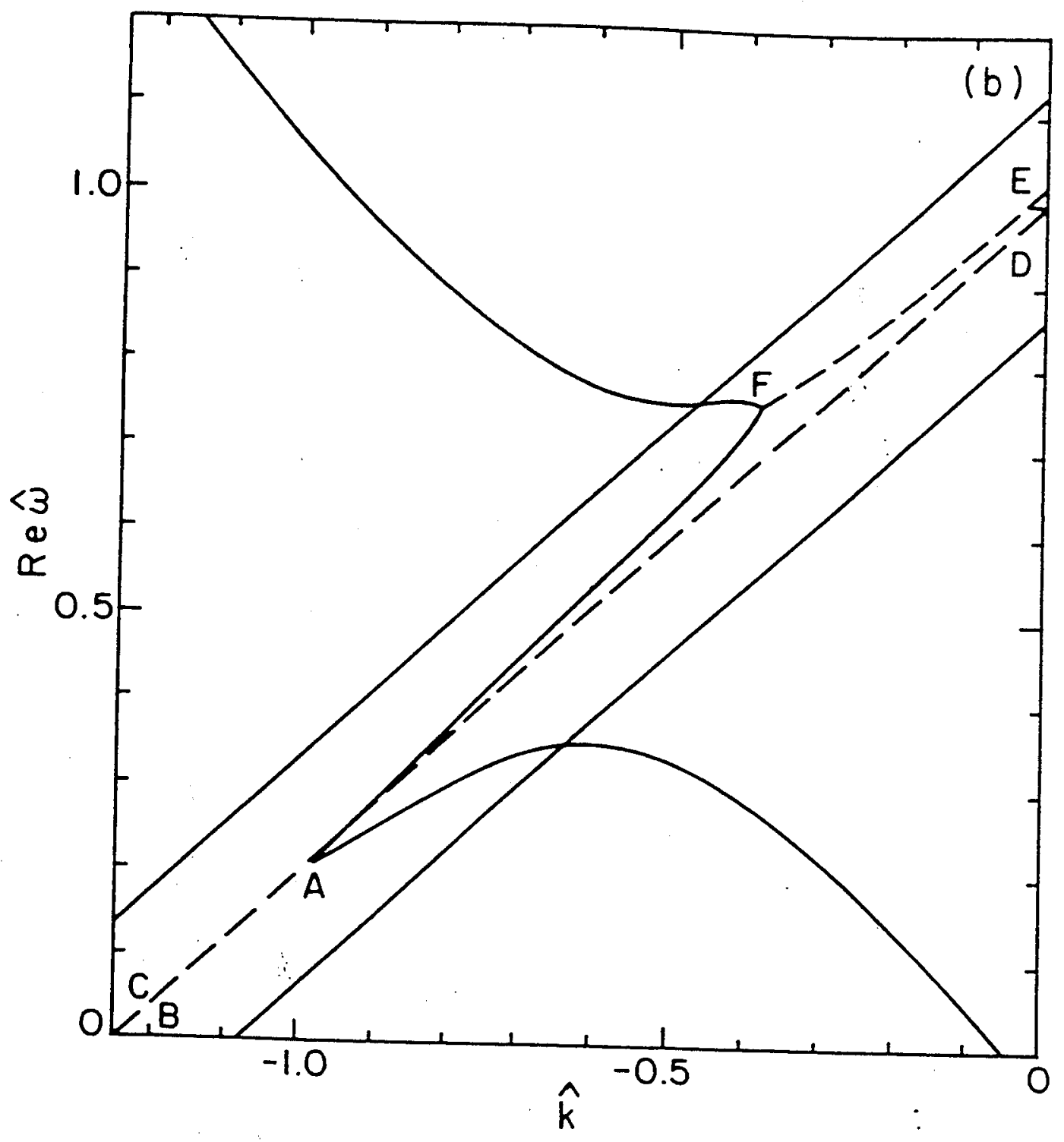

Figure 9(b)

Davies, Phys. Plasmas 


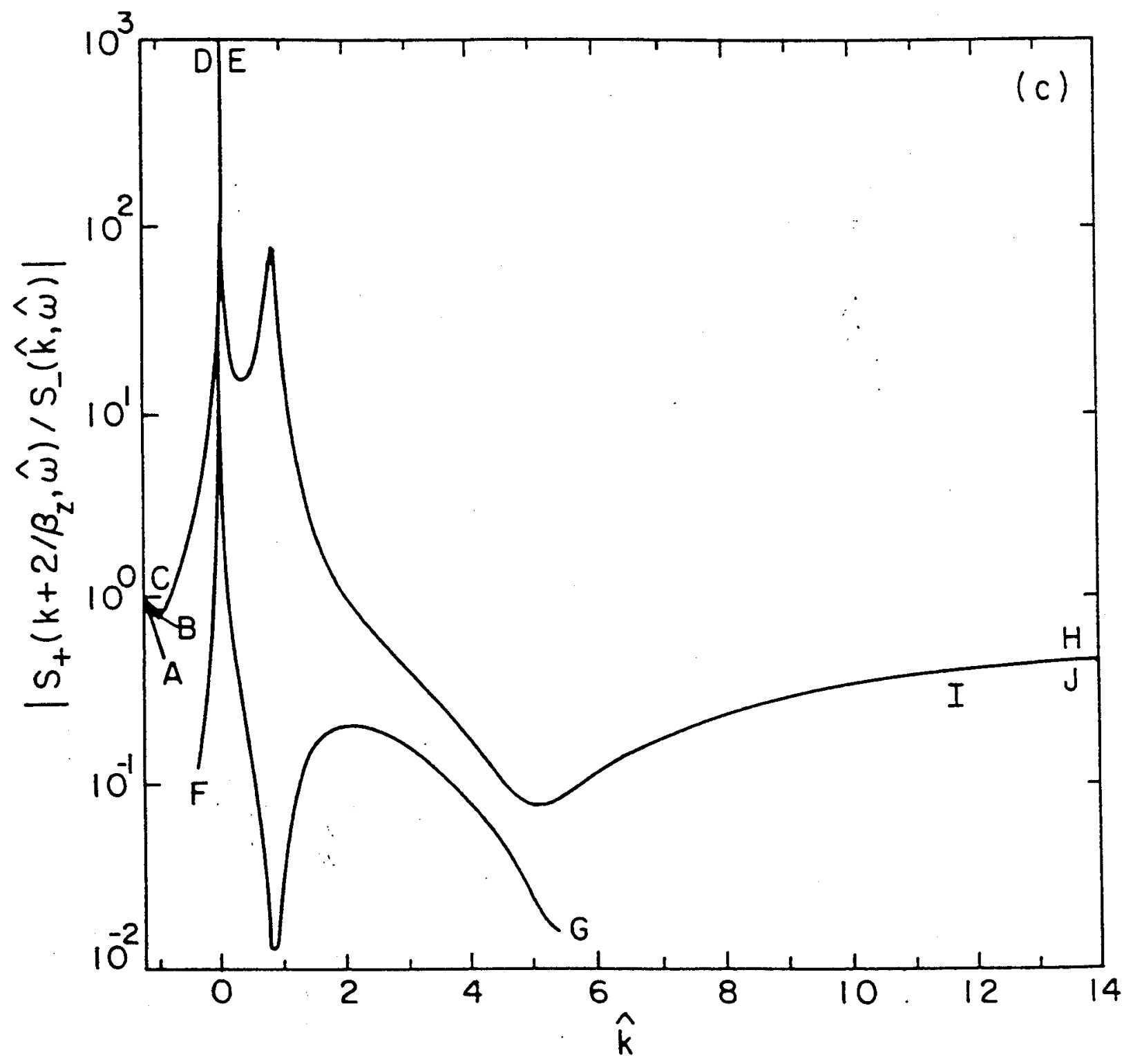

Figure 9(c)

Davies, Phys. Plasmas 


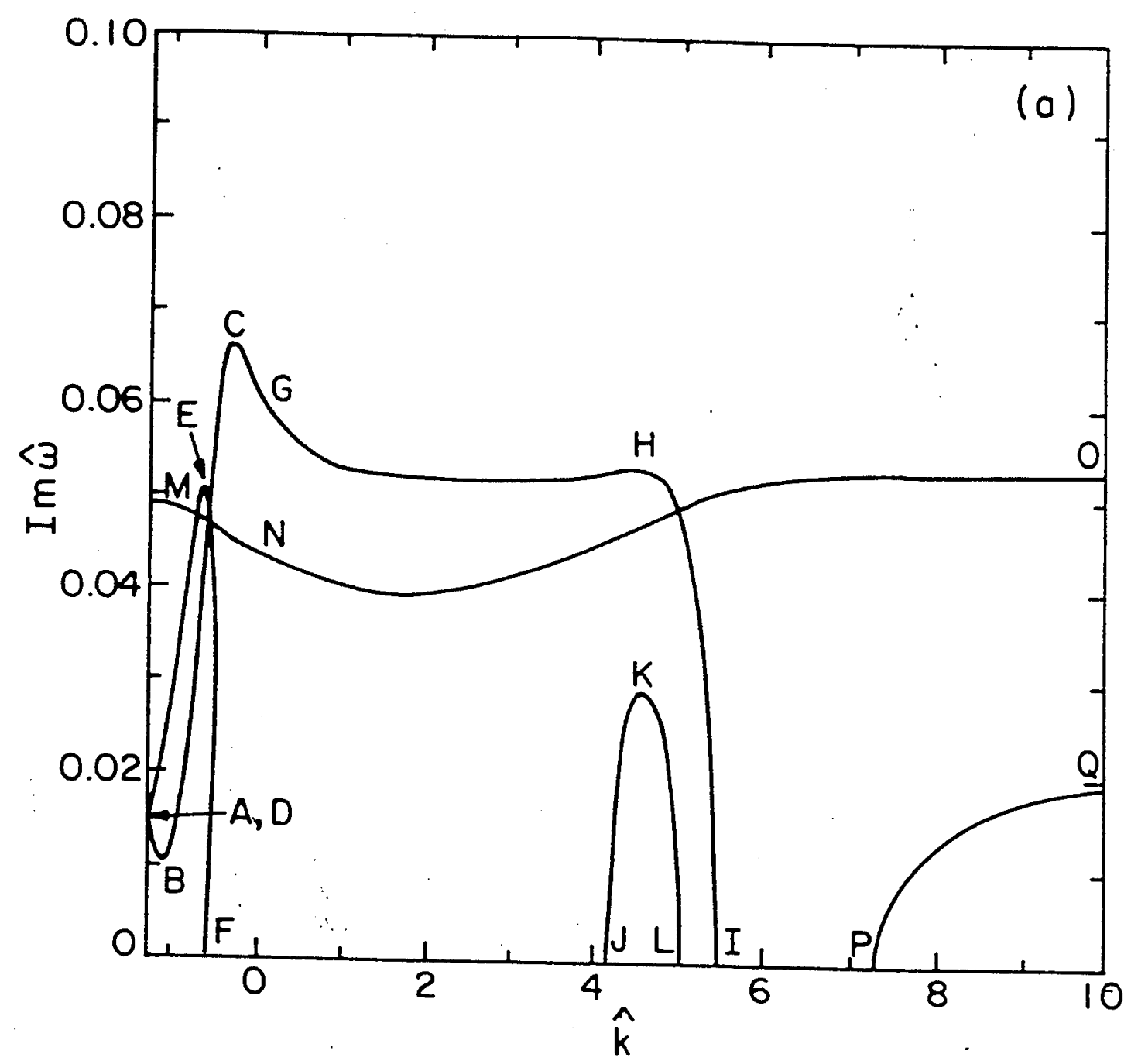

Figure 10(a)

Davies, Phys. Plissmas 


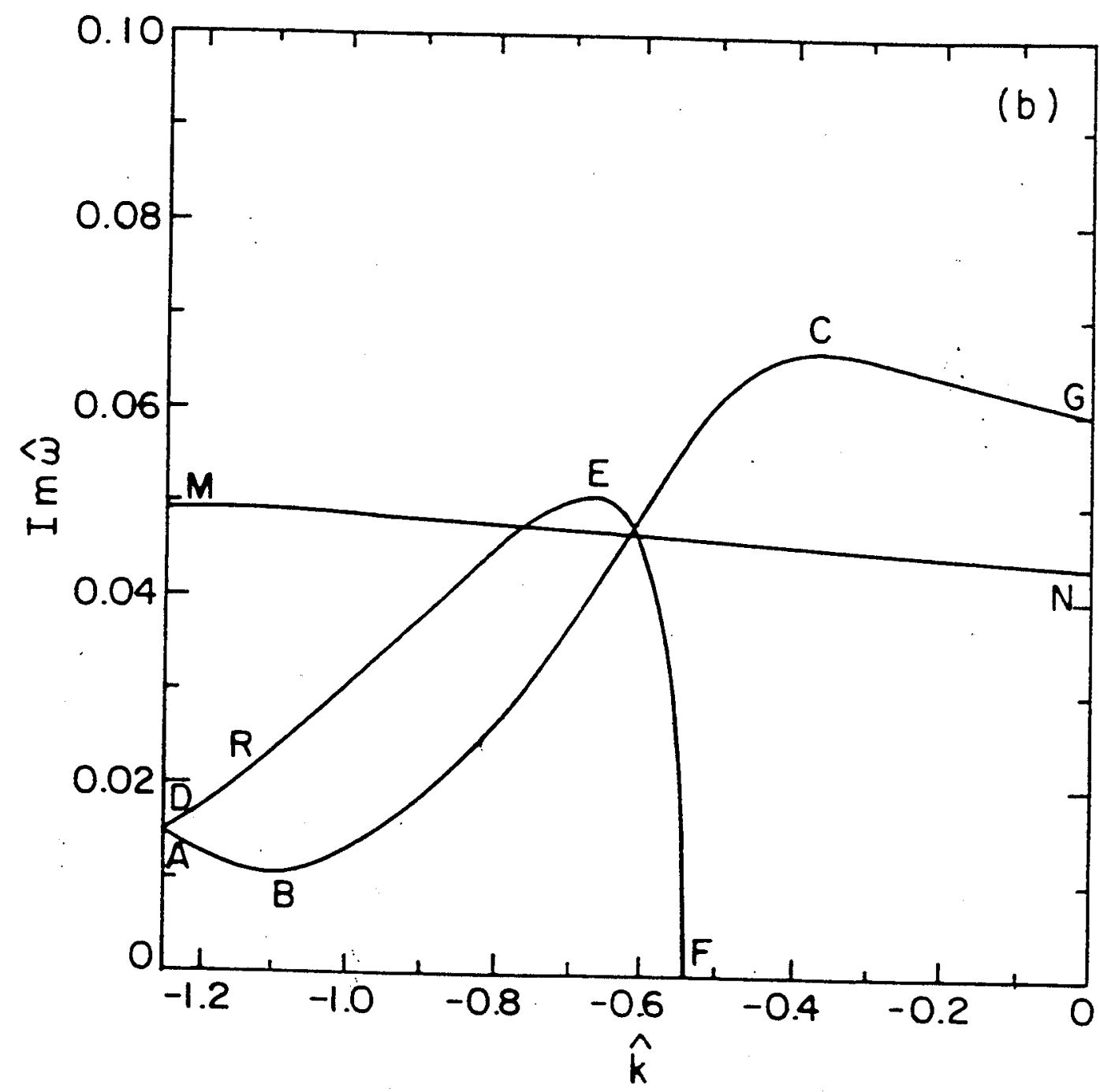

Figure 10(b)

Davies, Phys. Plasmas 


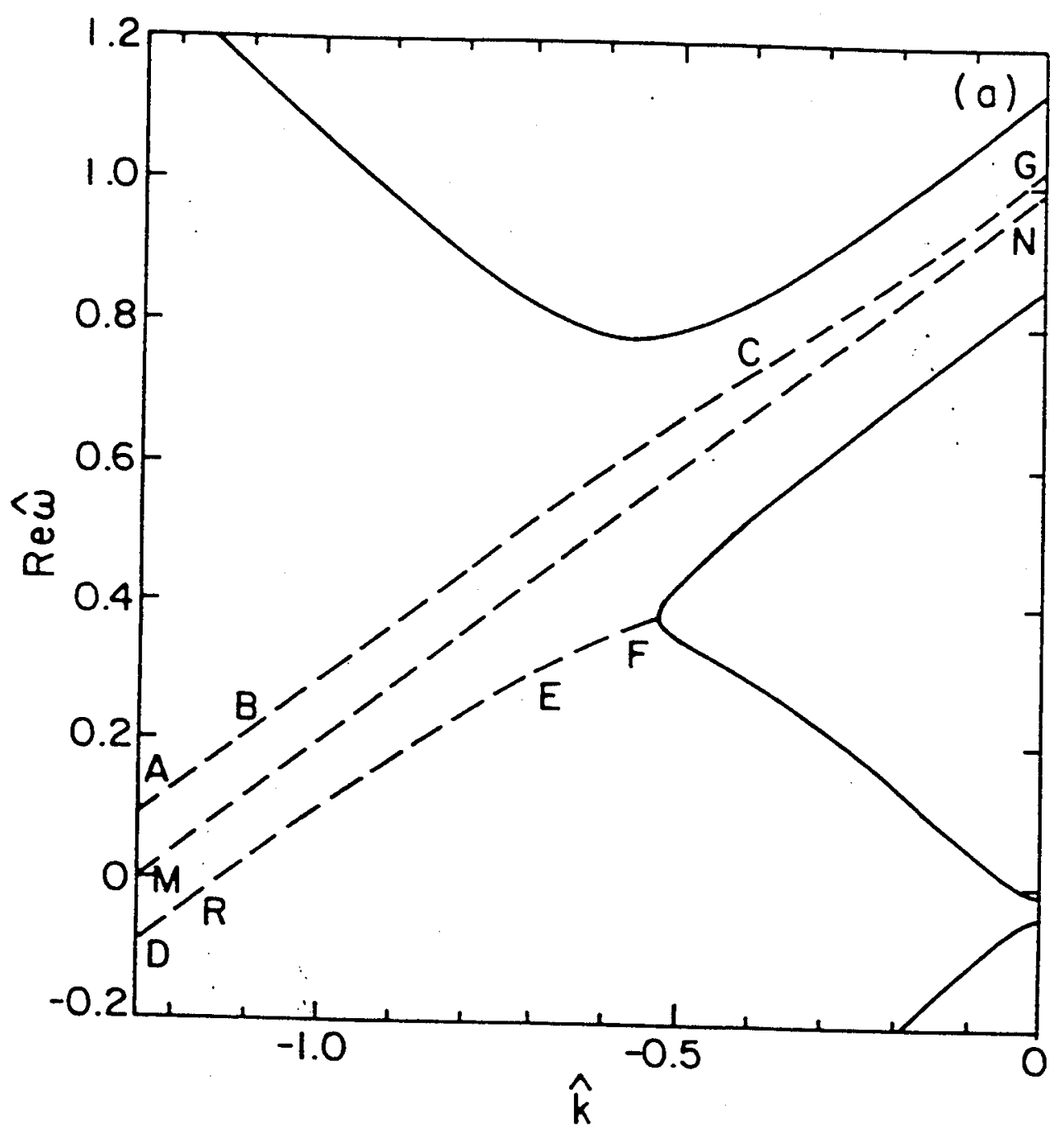

Figure 11(a)

Davies, Phys. Plasmas 


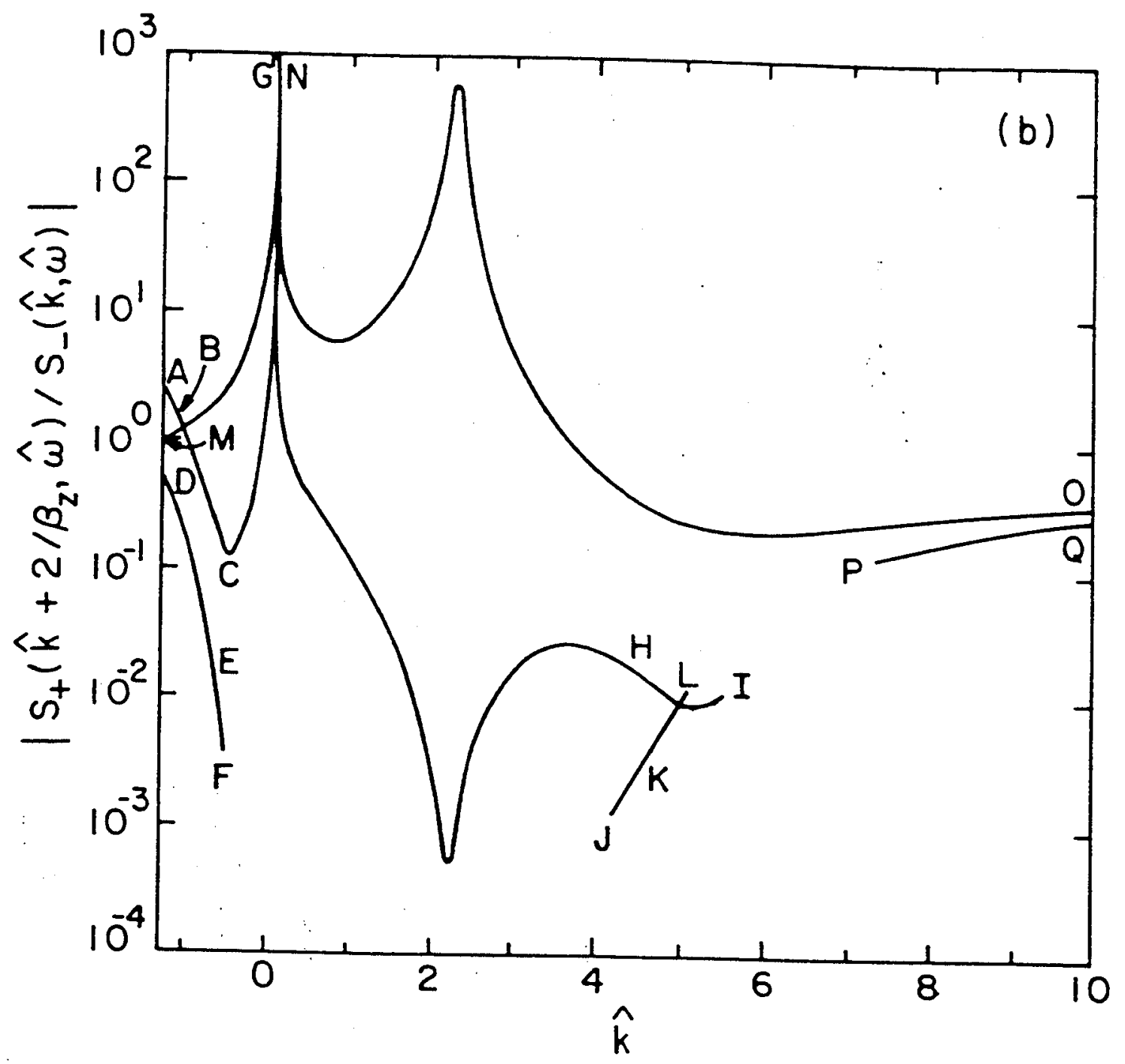

Figure 11(b)

Davies, Phys. Plasmas 


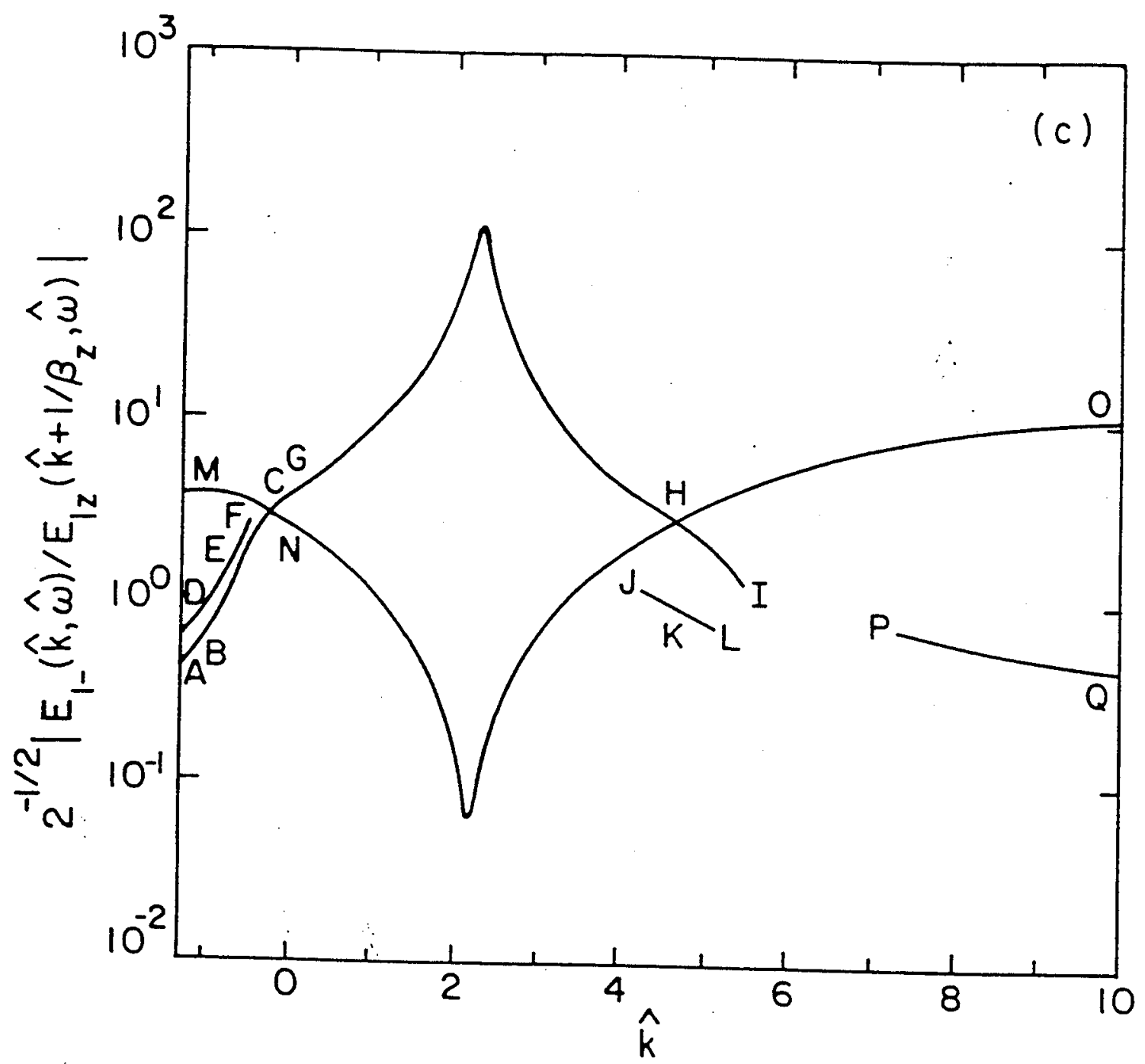

Figure 11(c)

Davies, Phys. Plasmas 


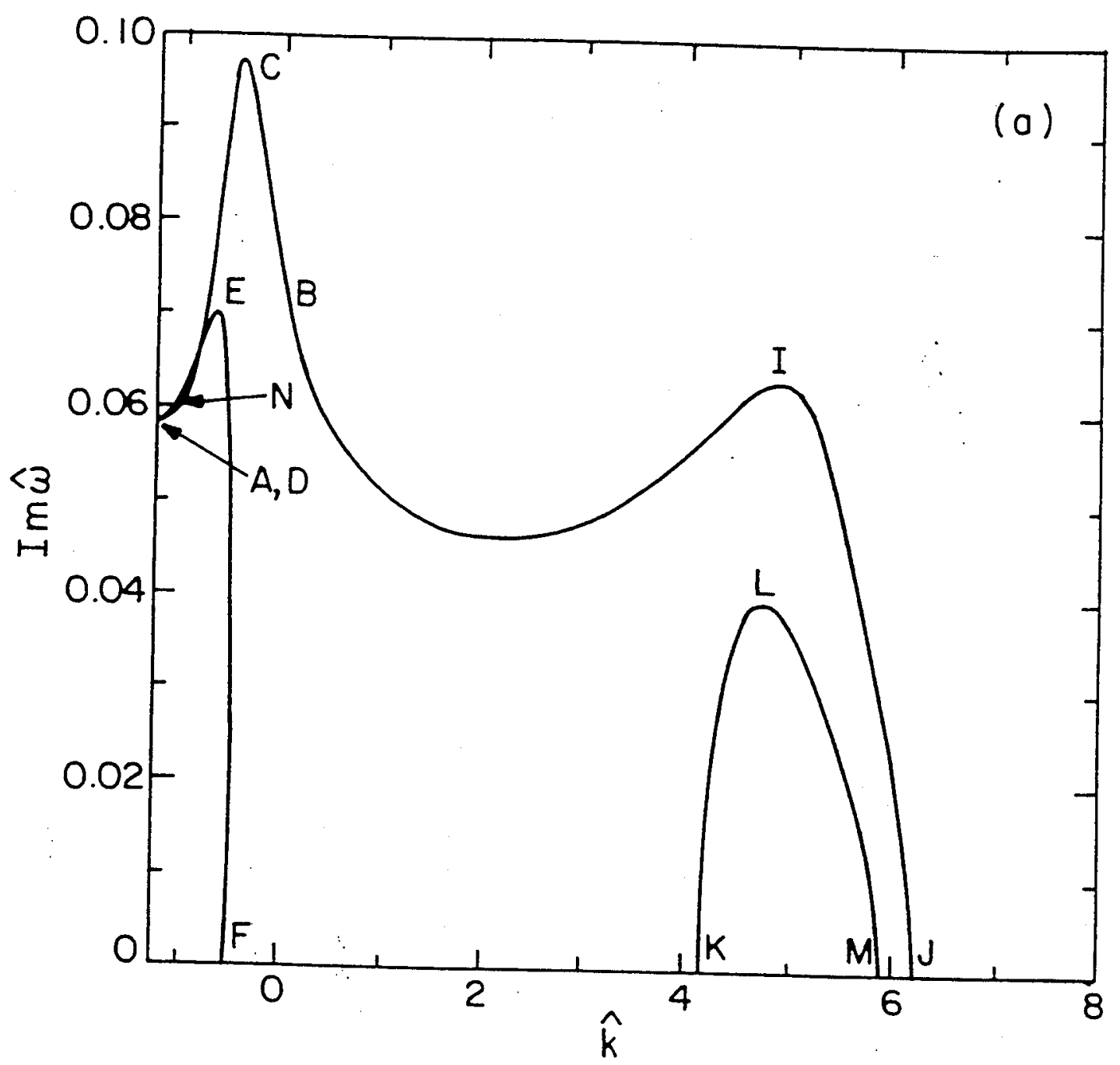

Figure 12(a)

Davies, Phys. Plasmas 


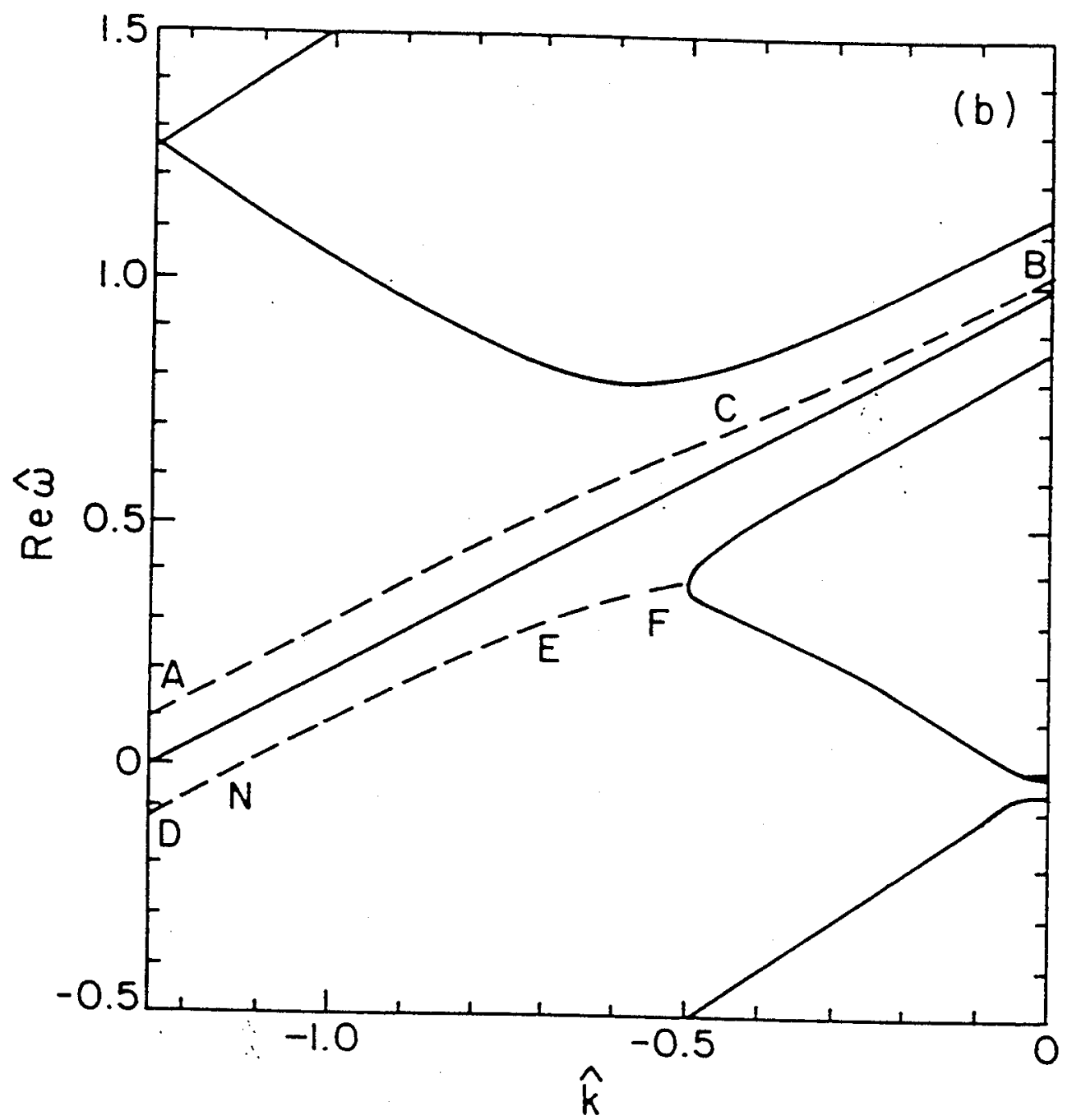

Figure 12(b)

Davies, Phys. Plasmas 


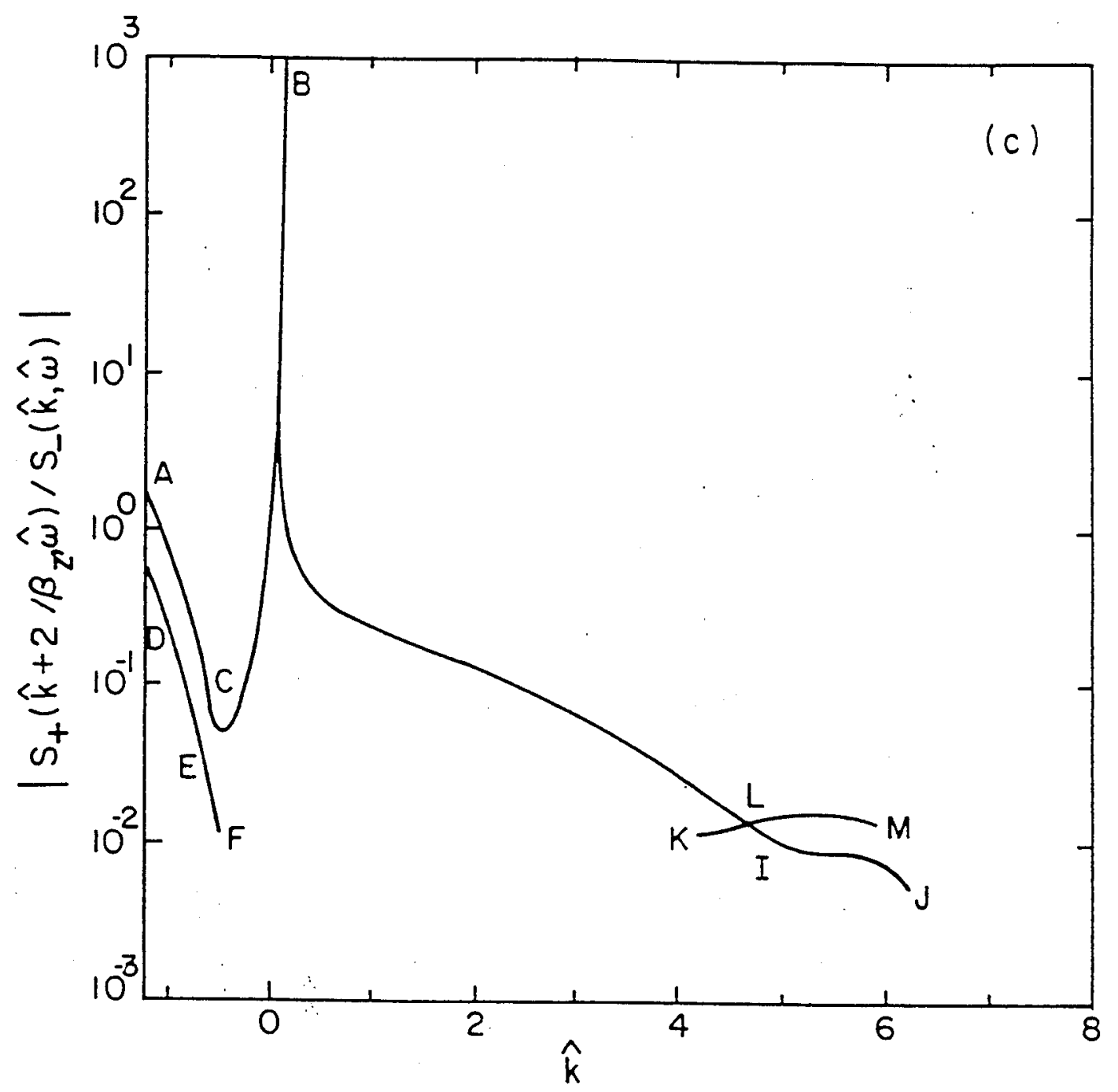

Figure 12(c)

Davies, Phys. Plasmas 


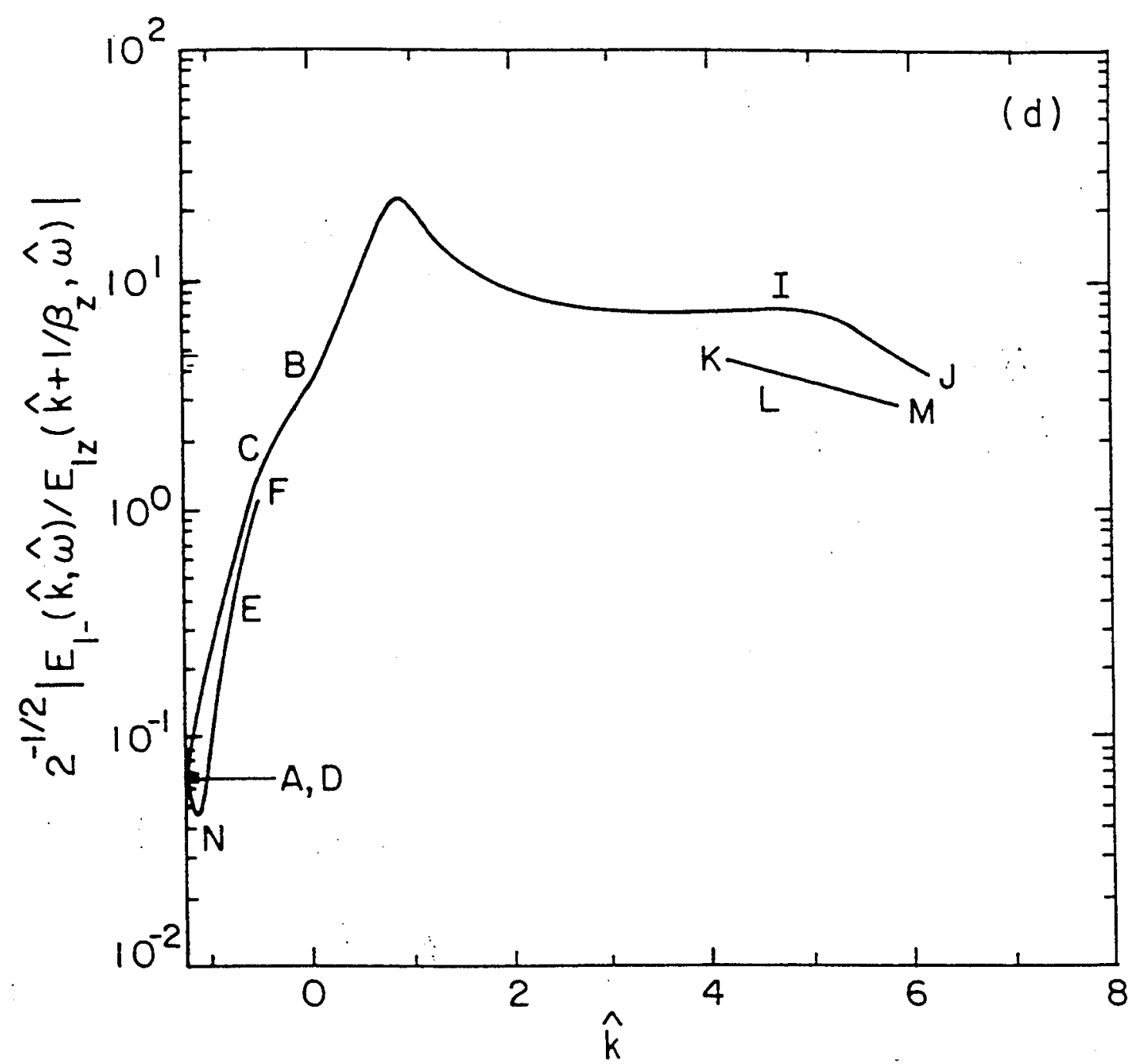

Figure 12(d)

Davies, Phys. Plasmas 